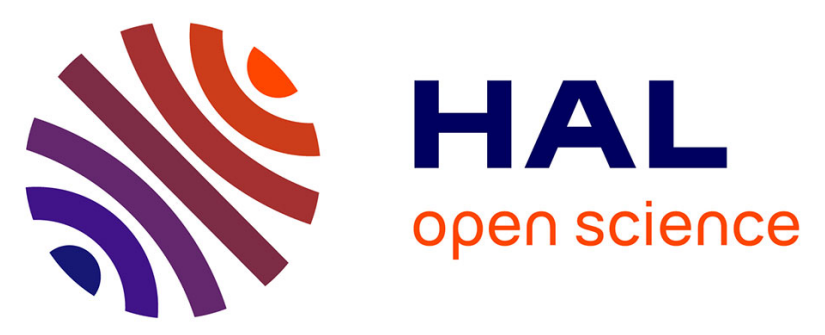

\title{
A posteriori estimates distinguishing the error components and adaptive stopping criteria for numerical approximations of parabolic variational inequalities
}

\author{
Jad Dabaghi, Vincent Martin, Martin Vohralík
}

\section{- To cite this version:}

Jad Dabaghi, Vincent Martin, Martin Vohralík. A posteriori estimates distinguishing the error components and adaptive stopping criteria for numerical approximations of parabolic variational inequalities. Computer Methods in Applied Mechanics and Engineering, 2020, 367, pp.113105. 10.1016/j.cma.2020.113105 . hal-02274493v3

\section{HAL Id: hal-02274493 \\ https://hal.science/hal-02274493v3}

Submitted on 29 Apr 2020

HAL is a multi-disciplinary open access archive for the deposit and dissemination of scientific research documents, whether they are published or not. The documents may come from teaching and research institutions in France or abroad, or from public or private research centers.
L'archive ouverte pluridisciplinaire HAL, est destinée au dépôt et à la diffusion de documents scientifiques de niveau recherche, publiés ou non, émanant des établissements d'enseignement et de recherche français ou étrangers, des laboratoires publics ou privés. 


\title{
A posteriori estimates distinguishing the error components and adaptive stopping criteria for numerical approximations of parabolic variational inequalities *
}

\author{
Jad Dabaghi ${ }^{\dagger \ddagger} \quad$ Vincent Martin ${ }^{\S} \quad$ Martin Vohralík ${ }^{\dagger \ddagger}$
}

April 29, 2020

\begin{abstract}
We consider in this paper a model parabolic variational inequality. This problem is discretized with conforming Lagrange finite elements of order $p \geq 1$ in space and with the backward Euler scheme in time. The nonlinearity coming from the complementarity constraints is treated with any semismooth Newton algorithm and we take into account in our analysis an arbitrary iterative algebraic solver. In the case $p=1$, when the system of nonlinear algebraic equations is solved exactly, we derive an a posteriori error estimate on both the energy error norm and a norm approximating the time derivative error. When $p \geq 1$, we provide a fully computable and guaranteed a posteriori estimate in the energy error norm which is valid at each step of the linearization and algebraic solvers. Our estimate, based on equilibrated flux reconstructions, also distinguishes the discretization, linearization, and algebraic error components. We build an adaptive inexact semismooth Newton algorithm based on stopping the iterations of both solvers when the estimators of the corresponding error components do not affect significantly the overall estimate. Numerical experiments are performed with the semismooth Newton-min algorithm and the semismooth Newton-Fischer-Burmeister algorithm in combination with the GMRES iterative algebraic solver to illustrate the strengths of our approach.
\end{abstract}

Keywords: parabolic variational inequality, complementarity condition, semismooth Newton method, algebraic solver, a posteriori error estimate, adaptivity, stopping criterion

\section{Introduction}

Let $\Omega \subset \mathbb{R}^{2}$ be a polygonal domain and let $T>0$ denote a final simulation time. Let $H^{1}(\Omega)$ be the space of $L^{2}$ functions on the domain $\Omega$ which admit a weak gradient in $\left[L^{2}(\Omega)\right]^{2}$ and $H_{0}^{1}(\Omega)$ its zero-trace subspace. Consider the affine space $H_{g}^{1}(\Omega):=\left\{v \in H^{1}(\Omega), v=g\right.$ on $\left.\partial \Omega\right\}$, where $g$ is a positive constant, and denote the dual space of $H_{0}^{1}(\Omega)$ by $H^{-1}(\Omega)$, with the duality pairing $\langle\cdot, \cdot\rangle$. Consider a bilinear continuous form $a(\cdot, \cdot):\left[H^{1}(\Omega)\right]^{2} \times\left[H^{1}(\Omega)\right]^{2} \rightarrow \mathbb{R}$, coercive on $\left[H_{0}^{1}(\Omega)\right]^{2}$. Let $\mathcal{K}_{g}$ be a nonempty closed convex subset of $H_{g}^{1}(\Omega) \times H_{0}^{1}(\Omega)$ and let $\mathcal{K}_{g}^{\mathrm{t}}$ be its evolutive-in-time version

$$
\mathcal{K}_{g}^{\mathrm{t}}:=\left\{\boldsymbol{v} \in L^{2}\left(0, T ; H_{g}^{1}(\Omega)\right) \times L^{2}\left(0, T ; H_{0}^{1}(\Omega)\right), \boldsymbol{v}(t) \in \mathcal{K}_{g} \text { a.e. in }\right] 0, T[\} .
$$

We consider the following parabolic variational inequality: for the data $\boldsymbol{f}:=\left(f_{1}, f_{2}\right) \in\left[L^{2}\left(0, T ; L^{2}(\Omega)\right)\right]^{2}$ and the initial condition $\boldsymbol{u}^{0}=\left(u_{1}^{0}, u_{2}^{0}\right) \in \mathcal{K}_{g}$, find $\boldsymbol{u}=\left(u_{1}, u_{2}\right) \in \mathcal{K}_{g}^{\mathrm{t}}$ such that $\partial_{t} \boldsymbol{u} \in\left[L^{2}\left(0, T ; H^{-1}(\Omega)\right)\right]^{2}$

\footnotetext{
*This project has received funding from the European Research Council (ERC) under the European Union's Horizon 2020 research and innovation program (grant agreement No 647134 GATIPOR).

${ }^{\dagger}$ Inria, 2 rue Simone Iff, 75589 Paris, France

‡Université Paris-Est, CERMICS (ENPC), 77455 Marne-la-Vallée 2, France

§Sorbonne universités, Université de technologie de Compiègne (UTC), LMAC, CS 60319, 60203 Compiègne Cedex
} 
and such that for all $\boldsymbol{v} \in \mathcal{K}_{g}^{\mathrm{t}}$

$$
\begin{aligned}
& \int_{0}^{T}\left\langle\partial_{t} \boldsymbol{u}, \boldsymbol{v}-\boldsymbol{u}\right\rangle(t) \mathrm{dt}+\int_{0}^{T} a(\boldsymbol{u}, \boldsymbol{v}-\boldsymbol{u})(t) \mathrm{dt} \geq \int_{0}^{T}(\boldsymbol{f}, \boldsymbol{v}-\boldsymbol{u})_{\Omega}(t) \mathrm{dt}, \\
& \boldsymbol{u}(0)=\boldsymbol{u}^{0}
\end{aligned}
$$

Problem (1.2) belongs to the wide class of parabolic variational inequalities of the first kind, see Glowinski [1] and Lions [2] for a general introduction. These have attracted a recent interest in a wide variety of applications; we mention the obstacle problems in mechanics [2, 3, 4], the problems in modeling pricing of American options [5, 6], the Stefan problem [7], the $\mathrm{CO}_{2}$ sequestration process [8], the mould filling [9], and the underground storage of radioactive waste [10]. Though the existence and uniqueness of a weak solution $\boldsymbol{u} \in \mathcal{K}_{g}^{\mathrm{t}}$ for (1.2) is classical, see [2] and the references therein, the numerical analysis of parabolic variational inequalities is very challenging.

A numerical discretization of problem (1.2) gives rise at each time step $n$ to a system of algebraic variational inequalities. This can be written as a system of linear equalities and complementarity constrains of the form

$$
\begin{aligned}
& \mathcal{S}^{n}\left(\boldsymbol{X}_{h}^{n}\right)=0, \\
& \mathcal{F}^{n}\left(\boldsymbol{X}_{h}^{n}\right) \geq 0, \quad \mathcal{G}^{n}\left(\boldsymbol{X}_{h}^{n}\right) \geq 0, \quad \mathcal{F}^{n}\left(\boldsymbol{X}_{h}^{n}\right) \cdot \mathcal{G}^{n}\left(\boldsymbol{X}_{h}^{n}\right)=0,
\end{aligned}
$$

where $\mathcal{S}^{n}, \mathcal{F}^{n}$, and $\mathcal{G}^{n}$ are affine operators and $\boldsymbol{X}_{h}^{n} \in \mathbb{R}^{m}, m \geq 1$, is the unknown vector of degrees of freedom. Among the spectrum of methods for their solution, let us mention the interior point method [11], the active set strategy [12], and the primal-dual active set strategy together with the family of semismooth Newton methods, see $[13,14,15,16,17,18,19,20]$ and the references therein.

The principle of any semismooth Newton linearization method is to approximate the solution of the nonlinear system (1.3) by an iterative procedure requesting to solve on each step a system of linear algebraic equations. The term "semismooth" describes here the particularity of this Newton linearization, which has to be able to cope with the fact that the system (1.3) is not differentiable everywhere, because of the algebraic inequalities in the constraints. More precisely, from an initial guess $\boldsymbol{X}_{h}^{n, 0} \in \mathbb{R}^{m}$, a semismooth Newton linearization requires to solve at each step $k \geq 1$ the system of linear algebraic equations

$$
\mathbb{A}^{n, k-1} \boldsymbol{X}_{h}^{n, k}=\boldsymbol{F}^{n, k-1},
$$

where the matrix $\mathbb{A}^{n, k-1} \in \mathbb{R}^{m, m}$ and the vector $\boldsymbol{F}^{n, k-1} \in \mathbb{R}^{m}$ are constructed from $\boldsymbol{X}_{h}^{n, k-1} \in \mathbb{R}^{m}$.

Solving (1.4) with a direct method may be very expensive. A popular approach to speed up the computation is to employ an inexact algebraic solver. At each iterative linear algebraic step $i \geq 0$ and each linearization step $k \geq 1$, this gives rise to a residual vector $\boldsymbol{R}_{h}^{n, k, i} \in \mathbb{R}^{m}$ defined by

$$
\boldsymbol{R}_{h}^{n, k, i}:=\boldsymbol{F}^{n, k-1}-\mathbb{A}^{n, k-1} \boldsymbol{X}_{h}^{n, k, i}
$$

In the present work, we focus on answering the following questions: To which precision should the linear system (1.4) be approximated? To which precision should the nonlinear non-differentiable system (1.3) be approximated? Can we estimate the total error, as well as the various error components (discretization, linearization, algebraic) of the overall numerical approximation of the exact solution $\boldsymbol{u}$ of (1.2)? Can we reduce the typical number of iterations of both linearization and algebraic solvers?

Our key tool to propose answers to the above questions is the a posteriori error analysis. An important amount of work has been performed in the recent past on a posteriori error estimates for variational inequalities, see Ainsworth and Oden [21] and Verfürth [22] for a general introduction. For the elliptic setting, we can mention the contributions $[23,24,25,26]$, where typically $\mathbb{P}_{1}$ discretizations are addressed. In contrast to these references, in Bürg and Schröder [27] and Dabaghi et al. [28], a $\mathbb{P}_{p}$ conforming finite element discretization is considered. Moreover, in [28], three components of the error are distinguished: the discretization error, the semismooth linearization error, and the iterative algebraic error.

In the context of parabolic problems, a posteriori analysis has also received a significant attention over the past decade. For parabolic equations, we mention Verfürth [29], Bernardi, Bergham, and Mghazli [30], and Ern, Smears, and Vohralík [31, 32], where in particular in [31], local efficiency in space and in time for the estimators is proven. For parabolic variational inequalities, the edifice seems still under construction. We 
can mention Moon, Nochetto, Petersdorff, and Zhang [33] for a study of the Black-Scholes model, Achdou, Hecht, and Pommier [3] for a study of the parabolic obstacle problem, and Gimperlein and Stocek [34] for a large variety of parabolic variational inequalities. In the present work, we follow the methodology of [31] and [28] to derive a posteriori error estimates with distinction of each component of the error. In particular, this enables us to define adaptive stopping criteria for nonlinear semismooth and linear algebraic solvers, which is new to the best of our knowledge. Importantly, it enables to save many unnecessary iterations.

To exemplify our approach, we consider the system of unsteady parabolic variational inequalities, as an extension of the stationary model problem studied in $[35,36,28]$, see $(2.1)$ below. Two important difficulties arise for the a posteriori analysis in this setting:

1) Denote by $\boldsymbol{u}_{h \tau}^{k, i}:=\left(u_{1 h \tau}^{k, i}, u_{2 h \tau}^{k, i}\right)$ the space-time numerical approximation, where the indices $k, i$ indicate the presence of inexact linearization and algebraic solvers and where $\boldsymbol{u}_{h \tau}^{k, i}$ is piecewise affine and continuous in time and piecewise polynomial of degree $p$ and continuous for each variable in space. Because of the use of polynomials of order $p \geq 2$, and because of the incomplete nonlinear and algebraic convergences in inexact schemes (even when $p=1), \boldsymbol{u}_{h \tau}^{k, i}$ is nonconforming in the sense that $\boldsymbol{u}_{h \tau}^{k, i} \notin \mathcal{K}_{g}^{\mathrm{t}}\left(u_{1 h \tau}^{k, i} \geq u_{2 h \tau}^{k, i}\right.$ does not hold everywhere in $\Omega$ for the model problem, see (2.1) below). The same phenomenon occurs for $\lambda_{h \tau}^{k, i}$ that denotes the discrete counterpart of the Lagrange multiplier $\lambda$. The conformity only occurs in the particular case $p=1$ and when both linearization and algebraic solvers have converged.

2) We cannot easily provide, as for the parabolic heat equation [31], an a posteriori upper bound for the time derivative $\left\|\partial_{t}\left(\boldsymbol{u}-\boldsymbol{u}_{h \tau}^{k, i}\right)\right\|_{\left[L^{2}\left(0, T ; H^{-1}(\Omega)\right)\right]^{2}}$. To tackle this difficulty at least for $p=1$ and exact solvers, where we simply denote $\boldsymbol{u}_{h \tau}=\boldsymbol{u}_{h \tau}^{k, i}$ the numerical approximation, we construct an element $\boldsymbol{z} \in \mathcal{K}_{g}^{\mathrm{t}}$ such that $\|\boldsymbol{u}-\boldsymbol{z}\|_{\left[L^{2}\left(0, T ; H_{0}^{1}(\Omega)\right)\right]^{2}}$ is closely linked to $\left\|\partial_{t}\left(\boldsymbol{u}-\boldsymbol{u}_{h \tau}\right)\right\|_{\left[L^{2}\left(0, T ; H^{-1}(\Omega)\right)\right]^{2}}$ and such that the a posteriori error estimate holds as

$$
\left\|\boldsymbol{u}-\boldsymbol{u}_{h \tau}\right\|_{\left[L^{2}\left(0, T ; H_{0}^{1}(\Omega)\right)\right]^{2}}^{2}+\|\boldsymbol{u}-\boldsymbol{z}\|_{\left[L^{2}\left(0, T ; H_{0}^{1}(\Omega)\right)\right.}^{2}+\left\|\left(\boldsymbol{u}-\boldsymbol{u}_{h \tau}\right)(\cdot, T)\right\|_{L^{2}(\Omega)}^{2} \leq\left(\eta\left(\boldsymbol{u}_{h \tau}\right)\right)^{2},
$$

with $\eta\left(\boldsymbol{u}_{h \tau}\right)$ a fully computable a posteriori error estimate, only depending on the approximate solution $\boldsymbol{u}_{h \tau}$.

In this contribution, we first present the model problem, its weak formulation, and its discretization with the backward Euler scheme in time and the conforming $\mathbb{P}_{p}(p \geq 1)$ finite element method in space which takes the form (1.3). Then, we present the concept of inexact semismooth Newton methods to approximate the solution of our system of algebraic inequalities at each time step. Next, we provide the a posteriori analysis following the approach of the equilibrated flux reconstructions. In particular, we derive an a posteriori error estimate for the linear finite elements $(p=1)$ at each time step $n$, when the semismooth Newton solver as well as the algebraic iterative solver have converged. Then we can estimate the error as shown in (1.6). We next provide a second a posteriori error estimate in the energy norm, valid for any polynomial degree $p \geq 1$ and at each semismooth linearization iteration $k \geq 1$ and each iterative algebraic solver iteration $i \geq 0$. This estimate only bounds the first component on the left-hand side of (1.6), but distinguishes the different error components, namely the discretization error, the semismooth linearization error, the algebraic error, and the initial error, taking the form

$$
\left\|\boldsymbol{u}-\boldsymbol{u}_{h \tau}^{k, i}\right\|_{\left[L^{2}\left(0, T ; H_{0}^{1}(\Omega)\right)\right]^{2}} \leq \tilde{\eta}\left(\boldsymbol{u}_{h \tau}^{k, i}\right) \leq \eta_{\mathrm{disc}}^{k, i}+\eta_{\mathrm{lin}}^{k, i}+\eta_{\mathrm{alg}}^{k, i}+\eta_{\text {init }}
$$

This leads us to a proposition of an adaptive inexact semismooth Newton algorithm for parabolic variational inequalities which relies on a posteriori stopping criteria for the linear and nonlinear solvers. Finally, we present numerical experiments when $p=1$ and $p=2$ with semismooth Newton linearization algorithms, in particular the Newton-min and the Newton-Fischer-Burmeister ones, in combination with the GMRES algebraic solver, assessing the strengths of our approach. 


\section{Model problem and setting}

Let $\Omega \subset \mathbb{R}^{2}$ be a polygonal domain and $T>0$ be the final simulation time. The model problem we consider here is to find $u_{1}, u_{2}$, and $\lambda$ such that

$$
\left\{\begin{array}{llr}
\partial_{t} u_{1}-\mu_{1} \Delta u_{1}-\lambda=f_{1} & \text { in } & \Omega \times] 0, T[, \\
\partial_{t} u_{2}-\mu_{2} \Delta u_{2}+\lambda=f_{2} & \text { in } & \Omega \times] 0, T[, \\
u_{1}-u_{2} \geq 0, \quad \lambda \geq 0, \quad\left(u_{1}-u_{2}\right) \lambda=0 & \text { in } & \Omega \times] 0, T[ \\
u_{1}=g, \quad u_{2}=0 & \text { on } & \partial \Omega \times] 0, T[ \\
u_{1}(0)=u_{1}^{0}, \quad u_{2}(0)=u_{2}^{0}, \quad u_{1}^{0}-u_{2}^{0} \geq 0 & \text { in } & \Omega .
\end{array}\right.
$$

Here, the real coefficients $\mu_{1}$ and $\mu_{2}$ are supposed constant and strictly positive, and, for the sake of simplicity, we assume that the Dirichlet boundary condition $g>0$ is also a constant. The source term $\boldsymbol{f}:=\left(f_{1}, f_{2}\right)$ is supposed to belong to $\left[L^{2}\left(0, T ; L^{2}(\Omega)\right)\right]^{2}$. Finally, the initial conditions are supposed to satisfy $\boldsymbol{u}^{0}:=\left(u_{1}^{0}, u_{2}^{0}\right) \in H_{g}^{1}(\Omega) \times H_{0}^{1}(\Omega)$ and $u_{1}^{0}-u_{2}^{0} \geq 0$ a.e. in $\Omega$. The first two equations of $(2.1)$ are of parabolic type. The third line of (2.1) states linear complementarity conditions expressing that either $u_{1}-u_{2}>0$ and $\lambda=0$, or $u_{1}-u_{2}=0$ and $\lambda>0$. Observe that when $u_{1}-u_{2}>0$ and $\lambda=0$ everywhere in $\Omega \times] 0, T$, problem $(2.1)$ is equivalent to solving two separated heat equations. On the other hand, when $f_{1}$ and $f_{2}$ are independent of time and $\partial_{t} u_{1}=\partial_{t} u_{2}=0,(2.1)$ becomes the stationary contact problem between two membranes studied in $[35,37,36,28,19]$.

We define the sets

$$
\Lambda:=\left\{\chi \in L^{2}(\Omega), \chi \geq 0 \text { a.e. in } \Omega\right\} \quad \text { and } \quad \Psi:=L^{2}(0, T ; \Lambda) .
$$

We also introduce the nonempty closed convex set

$$
\mathcal{K}_{g}:=\left\{\left(v_{1}, v_{2}\right) \in H_{g}^{1}(\Omega) \times H_{0}^{1}(\Omega), v_{1}-v_{2} \geq 0 \text { a.e. in } \Omega\right\},
$$

as well as its evolutive-in-time version $\mathcal{K}_{g}^{\mathrm{t}}$ defined by (1.1). [-] For a set $\mathcal{O}$ of $\mathbb{R}^{2}$, we denote its Lebesgue measure by $|\mathcal{O}|$ and the $L^{2}(\mathcal{O})$ scalar product for $\boldsymbol{w}:=\left(w_{1}, w_{2}\right) \in\left[L^{2}(\mathcal{O})\right]^{2}$ by $\left(w_{1}, w_{2}\right)_{\mathcal{O}}:=\int_{\mathcal{O}} w_{1} w_{2} \mathrm{dx}$. We also use the notations $\left\|w_{1}\right\|_{\mathcal{O}}^{2}:=\left(w_{1}, w_{1}\right)_{\mathcal{O}},\|\boldsymbol{w}\|_{\mathcal{O}}^{2}:=\sum_{\alpha=1,2}\left\|w_{\alpha}\right\|_{\mathcal{O}}^{2}$. The compact notations

$$
a(\boldsymbol{u}, \boldsymbol{v}):=\sum_{\alpha=1}^{2} \mu_{\alpha}\left(\nabla u_{\alpha}, \nabla v_{\alpha}\right)_{\Omega}, \quad b(\boldsymbol{v}, \chi):=\left(\chi, v_{1}-v_{2}\right)_{\Omega}
$$

will be useful henceforth, where $\boldsymbol{u}=\left(u_{1}, u_{2}\right), \boldsymbol{v}=\left(v_{1}, v_{2}\right), a$ is continuous and coercive as described in the introduction, and $b$ is a continuous bilinear form on $\left[H^{1}(\Omega)\right]^{2} \times L^{2}(\Omega)$. In the sequel, boldface variables such as $\boldsymbol{v}$ will denote couples of variables like $\left(v_{1}, v_{2}\right)$. For $\boldsymbol{v} \in\left[H_{0}^{1}(\mathcal{O})\right]^{2}$ and $\boldsymbol{w} \in\left[L^{2}\left(0, T ; H_{0}^{1}(\Omega)\right)\right]^{2}$, we define the space energy and the space-time energy norms by

$$
\|\boldsymbol{v}\|_{\mathcal{O}}:=\left\{\sum_{\alpha=1}^{2} \mu_{\alpha}\left\|\boldsymbol{\nabla} v_{\alpha}\right\|_{\mathcal{O}}^{2}\right\}^{\frac{1}{2}}, \quad\|\boldsymbol{w}\|_{\Omega, T}:=\left\{\int_{0}^{T}\|\boldsymbol{w}\|_{\Omega}^{2}(t) \mathrm{dt}\right\}^{\frac{1}{2}} .
$$

The weak formulation of problem (2.1) is given by the parabolic variational inequality (1.2) and it is well-posed. To illustrate the construction of the numerical discretization in Section 3 below, let us also mention that alternatively, one could look for $\left(u_{1}, u_{2}, \lambda\right) \in L^{2}\left(0, T ; H_{g}^{1}(\Omega)\right) \times L^{2}\left(0, T ; H_{0}^{1}(\Omega)\right) \times \Psi$ such that $\partial_{t} u_{\alpha} \in L^{2}\left(0, T ; H^{-1}(\Omega)\right), \alpha=1,2$, and satisfying for almost all $\left.t \in\right] 0, T\left[\right.$ and for all $\left(v_{1}, v_{2}, \chi\right) \in$ $H_{0}^{1}(\Omega) \times H_{0}^{1}(\Omega) \times \Lambda$

$$
\begin{aligned}
& \left\langle\partial_{t} \boldsymbol{u}(t), \boldsymbol{v}\right\rangle+a(\boldsymbol{u}(t), \boldsymbol{v})-b(\boldsymbol{v}, \lambda(t))=(\boldsymbol{f}(t), \boldsymbol{v})_{\Omega}, \\
& b(\boldsymbol{u}(t), \chi-\lambda(t)) \geq 0 \\
& \boldsymbol{u}(0)=\boldsymbol{u}^{0} .
\end{aligned}
$$

The second line in (2.5) can be interpreted as a linear complementarity constraint, see a derivation in the case of a stationary problem in [36],

$$
\left(u_{1}-u_{2}\right)(t) \geq 0, \quad \lambda(t) \geq 0, \quad \lambda(t)\left(u_{1}-u_{2}\right)(t)=0 .
$$




\section{Discretization and semismooth Newton linearization}

Our discretization of (2.1) relies on the backward Euler scheme in time and on the conforming finite element method of degree $p \geq 1$ in space. For the convenience of the reader, we give in this section the main results concerning the discretization, without proofs. They are an extension to the case of parabolic inequalities and for any $p \geq 2$ of the results given in $[37,28]$ for a stationary problem. The details are provided in [38, Sec. 2.5].

\section{$3.1 \quad$ Setting}

For the time discretization, we introduce a division of the interval $[0, T]$ into subintervals $I_{n}:=\left[t_{n-1}, t_{n}\right]$, $1 \leq n \leq N_{\mathrm{t}}$, such that $0=t_{0}<t_{1}<\cdots<t_{N_{\mathrm{t}}}=T$. The time steps are denoted by $\Delta t_{n}:=t_{n}-t_{n-1}$, $n=1, \cdots, N_{\mathrm{t}}$. The space domain $\Omega$ is discretized with a conforming triangular mesh $\mathcal{T}_{h}$. The set of vertices of $\mathcal{T}_{h}$ is denoted by $\mathcal{V}_{h}$ and is partitioned into interior vertices $\mathcal{V}_{h}^{\mathrm{i}}$ and boundary vertices $\mathcal{V}_{h}^{\mathrm{e}}$. The vertices of an element $K \in \mathcal{T}_{h}$ are collected in the set $\mathcal{V}_{K}$. Denote by $h_{K}$ the diameter of a triangle $K$ and $h:=\max _{K \in \mathcal{T}_{h}} h_{K}$. For the vertex $\boldsymbol{a} \in \mathcal{V}_{h}$, let the patch $\omega_{h}^{\boldsymbol{a}} \subset \Omega$ be the domain made up of the elements of $\mathcal{T}_{h}$ that share $\boldsymbol{a}$. In the sequel, we use the discrete conforming space of piecewise polynomial and continuous functions

$$
X_{h}^{p}:=\left\{v_{h} \in \mathcal{C}^{0}(\bar{\Omega}) ;\left.v_{h}\right|_{K} \in \mathbb{P}_{p}(K) \forall K \in \mathcal{T}_{h}\right\} \subset H^{1}(\Omega),
$$

where $\mathbb{P}_{p}(K)$ stands for the set of polynomials of total degree less than or equal to $p$ on the element $K$. We also denote by $\mathcal{V}^{p}$ the set of the Lagrange nodes $\boldsymbol{x}_{l}$ and by $\mathcal{N}^{p}$ its cardinality. The internal nodes are collected in the set $\mathcal{V}^{p, \mathrm{i}}$ whose cardinality is $\mathcal{N}^{p, \mathrm{i}}$, and the boundary nodes are collected in the set $\mathcal{V}^{p, \mathrm{e}}$. The Lagrange basis functions of $X_{h}^{p}$ are denoted by $\left(\psi_{h, \boldsymbol{x}_{l}}\right)_{1 \leq l \leq \mathcal{N}^{p}}, \boldsymbol{x}_{l} \in \mathcal{V}^{p} ; \psi_{h, \boldsymbol{x}_{l}}$ takes value one in $\boldsymbol{x}_{l}$ and zero in all other Lagrange nodes. In the particular case $p=1$, the set $\mathcal{V}^{1}$ coincides with $\mathcal{V}_{h}$, and the Lagrange basis functions are the "hat" basis functions denoted by $\psi_{h, \boldsymbol{a}}, \boldsymbol{a} \in \mathcal{V}_{h}$. We also introduce the boundary-aware set and space

$$
X_{g h}^{p}:=\left\{v_{h} \in X_{h}^{p}, v_{h}=g \text { on } \partial \Omega\right\} \subset H_{g}^{1}(\Omega), \quad X_{0 h}^{p}:=X_{h}^{p} \cap H_{0}^{1}(\Omega),
$$

and the convex set

$$
\mathcal{K}_{g h}^{p}:=\left\{\boldsymbol{v}_{h}=\left(v_{1 h}, v_{2 h}\right) \in X_{g h}^{p} \times X_{0 h}^{p}, v_{1 h}\left(\boldsymbol{x}_{l}\right)-v_{2 h}\left(\boldsymbol{x}_{l}\right) \geq 0 \quad \forall \boldsymbol{x}_{l} \in \mathcal{V}^{p, i}\right\} .
$$

Recall the definition (2.2) and observe that $\mathcal{K}_{g h}^{1} \subset \mathcal{K}_{g}$ holds in the case $p=1$ but $\mathcal{K}_{g h}^{p} \not \subset \mathcal{K}_{g}$ for $p \geq 2$. For $\alpha=1,2$, let us introduce the piecewise constant in time functions $\tilde{f}_{\alpha} \in L^{2}\left(0, T ; L^{2}(\Omega)\right)$ such that

$$
\left.\left(\tilde{f}_{\alpha}\right)\right|_{I_{n}}:=\frac{1}{\Delta t_{n}} \int_{I_{n}} f_{\alpha}(t) \mathrm{dt}, \quad \text { and denote } \tilde{f}_{\alpha}^{n}:=\left.\left(\tilde{f}_{\alpha}\right)\right|_{I_{n}} \in L^{2}(\Omega), \tilde{\boldsymbol{f}}:=\left(\tilde{f}_{1}, \tilde{f}_{2}\right), \tilde{\boldsymbol{f}}^{n}:=\left(\tilde{f}_{1}^{n}, \tilde{f}_{2}^{n}\right) .
$$

\subsection{Discrete reduced problem and discrete saddle-point problem}

Let

$$
c_{n}\left(\boldsymbol{u}_{h}^{n}, \boldsymbol{v}_{h}\right):=\frac{1}{\Delta t_{n}} \sum_{\alpha=1}^{2}\left(u_{\alpha h}^{n}, v_{\alpha h}\right)_{\Omega}, \quad 1 \leq n \leq N_{\mathrm{t}} .
$$

Given $\boldsymbol{u}_{h}^{0} \in \mathcal{K}_{g h}^{p}$, the discrete reduced problem corresponding to (1.2) consists in searching for all $1 \leq n \leq N_{\mathrm{t}}$ $\boldsymbol{u}_{h}^{n} \in \mathcal{K}_{g h}^{p}$ such that for all $\boldsymbol{v}_{h} \in \mathcal{K}_{g h}^{p}$

$$
c_{n}\left(\boldsymbol{u}_{h}^{n}-\boldsymbol{u}_{h}^{n-1}, \boldsymbol{v}_{h}-\boldsymbol{u}_{h}^{n}\right)+a\left(\boldsymbol{u}_{h}^{n}, \boldsymbol{v}_{h}-\boldsymbol{u}_{h}^{n}\right) \geq\left(\tilde{\boldsymbol{f}}^{n}, \boldsymbol{v}_{h}-\boldsymbol{u}_{h}^{n}\right)_{\Omega}
$$

From the Lions-Stampacchia theorem [39], the discrete problem (3.3) admits a unique solution. Recall that when $p \geq 2, \boldsymbol{u}_{h}^{n}$ is typically nonconforming in the sense that $\boldsymbol{u}_{h}^{n} \notin \mathcal{K}_{g}$.

Following the methodology of $[28,35,26,27]$, we define the discrete scalar product for all $\left(w_{h}, v_{h}\right) \in$ $X_{h}^{p} \times X_{h}^{p}$,

$$
\left\langle w_{h}, v_{h}\right\rangle_{h}:= \begin{cases}\sum_{\boldsymbol{a} \in \mathcal{V}_{h}} w_{h}(\boldsymbol{a}) v_{h}(\boldsymbol{a}) \frac{\left|\omega_{h}^{\boldsymbol{a}}\right|}{3} & \text { if } \quad p=1, \\ \left(w_{h}, v_{h}\right)_{\Omega} & \text { if } \quad p \geq 2 .\end{cases}
$$


Then, knowing $\boldsymbol{u}_{h}^{n}$, the solution of (3.3), we define for $1 \leq n \leq N_{\mathrm{t}}$ and for all $\alpha=1,2$ the functions $\lambda_{\alpha h}^{n} \in X_{h}^{p}$ by

$$
\begin{aligned}
\left\langle\lambda_{\alpha h}^{n}, z_{\alpha h}\right\rangle_{h} & :=(-1)^{\alpha}\left[-\frac{1}{\Delta t_{n}}\left(u_{\alpha h}^{n}-u_{\alpha h}^{n-1}, z_{\alpha h}\right)_{\Omega}-\mu_{\alpha}\left(\nabla u_{\alpha h}^{n}, \nabla z_{\alpha h}\right)_{\Omega}+\left(\tilde{f}_{\alpha}^{n}, z_{\alpha h}\right)_{\Omega}\right] \forall z_{\alpha h} \in X_{0 h}^{p}, \\
\left\langle\lambda_{\alpha h}^{n}, \psi_{h, x_{l}}\right\rangle_{h} & :=0 \quad \forall \boldsymbol{x}_{l} \in \mathcal{V}^{p, e}
\end{aligned}
$$

Next, we define the discrete convex set

$$
\Lambda_{h}^{p}:=\left\{v_{h} \in X_{h}^{p} ;\left\langle v_{h}, \psi_{h, \boldsymbol{x}_{l}}\right\rangle_{h} \geq 0 \forall \boldsymbol{x}_{l} \in \mathcal{V}^{p, \mathrm{i}},\left\langle v_{h}, \psi_{h, \boldsymbol{x}_{l}}\right\rangle_{h}=0 \forall \boldsymbol{x}_{l} \in \mathcal{V}^{p, \mathrm{e}}\right\}
$$

Note that $\Lambda_{h}^{p} \not \subset \Lambda$ for $p \geq 2$. For linear finite elements $(p=1)$, one has

$$
\Lambda_{h}^{1}=\left\{v_{h} \in X_{0 h}^{1} ; v_{h}(\boldsymbol{a}) \geq 0 \forall \boldsymbol{a} \in \mathcal{V}_{h}^{\mathrm{i}}\right\}=\left\{v_{h} \in X_{0 h}^{1} ; v_{h} \geq 0\right\} \subset \Lambda .
$$

The following Lemma is a generalization to the case of parabolic inequality and with $p \geq 2$ of [37, Lemma 13].

Lemma 3.1. Let $1 \leq n \leq N_{\mathrm{t}}$ be a time step and $\boldsymbol{u}_{h}^{n} \in \mathcal{K}_{g h}^{p}$ be the solution of the reduced discrete problem (3.3). With the construction (3.5), the functions $\lambda_{1 h}^{n}$ and $\lambda_{2 h}^{n}$ defined by (3.5) coincide, and we set $\lambda_{h}^{n}:=\lambda_{1 h}^{n}=\lambda_{2 h}^{n}$. Furthermore, there holds $\lambda_{h}^{n} \in \Lambda_{h}^{p}$.

It will be useful to also consider the discrete formulation corresponding to problem (2.5). Given $\boldsymbol{u}_{h}^{0} \in$ $\mathcal{K}_{g h}^{p}$, it consists, for each $n=1 \cdots N_{\mathrm{t}}$, in searching $\left(\boldsymbol{u}_{h}^{n}, \lambda_{h}^{n}\right) \in\left[X_{g h}^{p} \times X_{0 h}^{p}\right] \times \Lambda_{h}^{p}$ such that for all $\left(\boldsymbol{v}_{h}, \chi_{h}\right) \in$ $\left[X_{0 h}^{p}\right]^{2} \times \Lambda_{h}^{p}$,

$$
\begin{gathered}
c_{n}\left(\boldsymbol{u}_{h}^{n}-\boldsymbol{u}_{h}^{n-1}, \boldsymbol{v}_{h}\right)+a\left(\boldsymbol{u}_{h}^{n}, \boldsymbol{v}_{h}\right)-\left\langle\lambda_{h}^{n}, v_{1 h}-v_{2 h}\right\rangle_{h}=\left(\tilde{\boldsymbol{f}}^{n}, \boldsymbol{v}_{h}\right)_{\Omega}, \\
\left\langle\chi_{h}-\lambda_{h}^{n}, u_{1 h}^{n}-u_{2 h}^{n}\right\rangle_{h} \geq 0 .
\end{gathered}
$$

We can now link formulations (3.3) and (3.8). The proof of the following lemma is a direct extension of [37, Lemma 13] in the case $p=1$.

Lemma 3.2. Let $1 \leq n \leq N_{\mathrm{t}}$ be a time step. For any solution $\left(\boldsymbol{u}_{h}^{n}, \lambda_{h}^{n}\right)$ of problem (3.8), $\boldsymbol{u}_{h}^{n}$ is a solution of problem (3.3). Conversely, for any solution $\boldsymbol{u}_{h}^{n}$ of problem (3.3), defining the function $\lambda_{h}^{n}:=\lambda_{\alpha h}^{n}, \alpha=1,2$, by $(3.5),\left(\boldsymbol{u}_{h}^{n}, \lambda_{h}^{n}\right)$ is a solution of problem (3.8).

From Lemma 3.2, we deduce that problem (3.8) admits a unique weak solution for each $n=1, \cdots, N_{\mathrm{t}}$. We finish this section by the following remark:

Remark 3.3. Taking in (3.8b) $\chi_{h}=0$ and next $\chi_{h}=2 \lambda_{h}^{n} \in \Lambda_{h}^{p}$ gives $\left\langle\lambda_{h}^{n}, u_{1 h}^{n}-u_{2 h}^{n}\right\rangle_{h}=0$. As $\boldsymbol{u}_{h}^{n} \in \mathcal{K}_{g h}^{p}$ and $\lambda_{h}^{n} \in \Lambda_{h}^{p}$, we obtain a discrete equivalent of the complementarity condition (2.6) valid for all polynomial degrees $p \geq 1$ :

$$
\begin{aligned}
& \left(u_{1 h}^{n}-u_{2 h}^{n}\right)\left(\boldsymbol{x}_{l}\right) \geq 0 \forall \boldsymbol{x}_{l} \in \mathcal{V}^{p, \mathrm{i}},\left\langle\lambda_{h}^{n}, \psi_{h, \boldsymbol{x}_{l}}\right\rangle_{h} \geq 0, \forall \boldsymbol{x}_{l} \in \mathcal{V}^{p, \mathrm{i}},\left\langle\lambda_{h}^{n}, \psi_{h, \boldsymbol{x}_{l}}\right\rangle_{h}=0 \forall \boldsymbol{x}_{l} \in \mathcal{V}^{p, \mathrm{e}}, \\
& \left\langle\lambda_{h}^{n}, u_{1 h}^{n}-u_{2 h}^{n}\right\rangle_{h}=0 .
\end{aligned}
$$

Note that for $p=1,(3.9)$ implies $u_{1 h}^{n} \geq u_{2 h}^{n}$ and $\lambda_{h}^{n} \geq 0$ everywhere, which means that $\boldsymbol{u}_{h} \in \mathcal{K}_{g}^{\mathrm{t}}$ and $\lambda_{h} \in \Lambda$ : the solution is conforming.

\subsection{Numerical resolution and discrete complementarity constraints}

Let $n$ be fixed in $\left\{1, \ldots, N_{\mathrm{t}}\right\}$. As in [28], we write in an algebraic form the discrete problem (3.8), using the expression (3.9) for the constraints. For the Lagrange muliplier, we will use the basis $\left(\Theta_{h, \boldsymbol{x}_{l}}\right)_{1 \leq l \leq \mathcal{N}^{p}}$ of $X_{h}^{p}$, dual to $\left(\psi_{h, \boldsymbol{x}_{l}}\right)_{1 \leq l \leq \mathcal{N}^{p}}$, given by

$$
\begin{aligned}
\left\langle\Theta_{h, x_{l}}, \psi_{h, x_{l}}\right\rangle_{h}=1 & \forall \boldsymbol{x}_{l} \in \mathcal{V}^{p}, \\
\left\langle\Theta_{h, x_{l}}, \psi_{h, x_{l}^{*}}\right\rangle_{h}=0 & \forall \boldsymbol{x}_{l^{*}} \in \mathcal{V}^{p}, \boldsymbol{x}_{l^{*}} \neq \boldsymbol{x}_{l} .
\end{aligned}
$$


Note that each vector $\Theta_{h, \boldsymbol{x}_{l}}$ of the dual basis can be determined by inverting a diagonal (lumped mass) matrix for $p=1$ and the finite element mass matrix for $p \geq 2$. Note also that the support of $\Theta_{h, \boldsymbol{x}_{l}}$ is typically not local. Importantly, all $\Theta_{h, \boldsymbol{x}_{l}}, \forall \boldsymbol{x}_{l} \in \mathcal{V}^{p, \mathrm{i}}$, belong to $\Lambda_{h}^{p}$, so using (3.9), $\lambda_{h}$ can be decomposed on the subset $\left(\Theta_{h, \boldsymbol{x}_{l}}\right)_{1 \leq l \leq \mathcal{N}^{p}, \mathrm{i}}$ of the basis $\left(\Theta_{h, \boldsymbol{x}_{l}}\right)_{1 \leq l \leq \mathcal{N}^{p}}$. Noting that $X_{g h}^{p}$ is decomposed as $X_{g h}^{p}=X_{0 h}^{p}+g$ (recall that $g>0$ is constant), the unknown piecewise polynomial functions can be decomposed as

$$
u_{1 h}^{n}=\sum_{l=1}^{\mathcal{N}^{p, \mathrm{i}}}\left(\boldsymbol{X}_{1 h}^{n}\right)_{l} \psi_{h, \boldsymbol{x}_{l}}+g, \quad u_{2 h}^{n}=\sum_{l=1}^{\mathcal{N}^{p, \mathrm{i}}}\left(\boldsymbol{X}_{2 h}^{n}\right)_{l} \psi_{h, \boldsymbol{x}_{l}}, \quad \lambda_{h}^{n}=\sum_{l=1}^{\mathcal{N}^{p, \mathrm{i}}}\left(\boldsymbol{X}_{3 h}^{n}\right)_{l} \Theta_{h, \boldsymbol{x}_{l}}
$$

so the algebraic vector of unknowns is $\left[\boldsymbol{X}_{h}^{n}\right]^{T}:=\left[\boldsymbol{X}_{1 h}^{n}, \boldsymbol{X}_{2 h}^{n}, \boldsymbol{X}_{3 h}^{n}\right]^{T} \in \mathbb{R}^{3 \mathcal{N}^{p, i}}$. The initial value $\boldsymbol{u}_{h}^{0} \in \mathcal{K}_{g h}^{p}$ is represented in the same way by $\left[\boldsymbol{X}_{h}^{0}\right]^{T}:=\left[\boldsymbol{X}_{1 h}^{0}, \boldsymbol{X}_{2 h}^{0}\right]^{T} \in \mathbb{R}^{2 \mathcal{N}^{p, i}}$. In algebraic form, (3.8a) reads $\mathbb{E}_{p}^{n} \boldsymbol{X}_{h}^{n}=$ $\boldsymbol{F}^{n}$, where $\mathbb{E}_{p}^{n} \in \mathbb{R}^{2 \mathcal{N}^{p, \mathrm{i}}, 3 \mathcal{N}^{p, \mathrm{i}}}$ is the rectangular matrix defined by

$$
\mathbb{E}_{p}^{n}:=\left[\begin{array}{ccc}
\mu_{1} \mathbb{S}+\frac{1}{\Delta t_{n}} \mathbb{M} & \mathbf{0} & -\mathbb{I}_{\mathrm{d}} \\
\mathbf{0} & \mu_{2} \mathbb{S}+\frac{1}{\Delta t_{n}} \mathbb{M} & +\mathbb{I}_{\mathrm{d}}
\end{array}\right],
$$

$\mathbb{I}_{\mathrm{d}} \in \mathbb{R}^{\mathcal{N}^{p, \mathrm{i}}, \mathcal{N}^{p, \mathrm{i}}}$ is the identity matrix, and the finite element mass and stiffness matrices $\mathbb{M}$ and $\mathbb{S}$ belonging to $\mathbb{R}^{\mathcal{N}^{p, \mathrm{i}}, \mathcal{N}^{p, \mathrm{i}}}$ are respectively defined by

$$
\mathbb{M}_{l, m}:=\left(\psi_{h, \boldsymbol{x}_{l}}, \psi_{h, \boldsymbol{x}_{m}}\right)_{\Omega}, \quad \mathbb{S}_{l, m}:=\left(\boldsymbol{\nabla} \psi_{h, \boldsymbol{x}_{l}}, \boldsymbol{\nabla} \psi_{h, \boldsymbol{x}_{m}}\right)_{\Omega}, \quad 1 \leq l, m \leq \mathcal{N}^{p, \mathrm{i}}
$$

The right-hand side vector $\boldsymbol{F}^{n}$ is defined by blocks $\left[\boldsymbol{F}^{n}\right]^{T}:=\left[\boldsymbol{F}_{1}^{n}, \boldsymbol{F}_{2}^{n}\right]^{T}$ as

$$
\left(\boldsymbol{F}_{\alpha}^{n}\right)_{l}:=\left(\tilde{f}_{\alpha}^{n}+\frac{1}{\Delta t_{n}} u_{\alpha h}^{n-1}, \psi_{h, \boldsymbol{x}_{l}}\right)_{\Omega}, \quad 1 \leq l \leq \mathcal{N}^{p, \mathrm{i}}, \quad \alpha=1,2 .
$$

Let $\mathbf{1}=(1,1, \cdots, 1)^{T} \in \mathbb{R}^{\mathcal{N}^{p, \mathrm{i}}}$. Expressing the complementarity constraints by (3.9) and using (3.10), the system (3.8) can be written for any $p \geq 1$ as: for $n=1, \cdots, N_{\mathrm{t}}$, given $\boldsymbol{X}_{h}^{n-1} \in \mathbb{R}^{2 \mathcal{N}^{p, \mathrm{i}}}$, search $\boldsymbol{X}_{h}^{n} \in \mathbb{R}^{3 \mathcal{N}^{p, \mathrm{i}}}$ such that

$$
\begin{aligned}
& \mathbb{E}_{p}^{n} \boldsymbol{X}_{h}^{n}=\boldsymbol{F}^{n}, \\
& \boldsymbol{X}_{1 h}^{n}+g \mathbf{1}-\boldsymbol{X}_{2 h}^{n} \geq 0, \quad \boldsymbol{X}_{3 h}^{n} \geq 0, \quad\left(\boldsymbol{X}_{1 h}^{n}+g \mathbf{1}-\boldsymbol{X}_{2 h}^{n}\right) \cdot \boldsymbol{X}_{3 h}^{n}=0 .
\end{aligned}
$$

Remark 3.4. Note that $\boldsymbol{u}_{h}^{n}$ is expressed in the Lagrange basis $\left(\psi_{h, \boldsymbol{x}_{l}}\right)_{1 \leq l<\mathcal{N}^{p, i},}$, while $\lambda_{h}^{n}$ is expressed with the dual basis $\left(\Theta_{h, \boldsymbol{x}_{l}}\right)_{1 \leq l \leq \mathcal{N}^{p}}$. It is also possible to express $\lambda_{h}^{n}$ in the Lagrange basis $\left(\psi_{h, \boldsymbol{x}_{l}}\right)_{1 \leq l \leq \mathcal{N}^{p}}$ of $X_{h}^{p}$, see [38, Sect. 2.5]. In such a case, the complementary constraints are expressed in a less convenient manner, with submatrices of the finite element mass matrix. Note also that the blocks $\mathbb{I}_{\mathrm{d}}$ in the matrix $\mathbb{E}_{p}^{n}$ are replaced by the mass matrix.

\subsection{Equivalent rewriting using C-functions}

The complementarity constraints $(3.12 \mathrm{~b})$ write as algebraic inequalities. We now rewrite them as nonlinear equalities. A function $f:\left(\mathbb{R}^{m}\right)^{2} \rightarrow \mathbb{R}^{m}, m \geq 1$, is called a $C$-function or a complementarity function, see $[16,17]$, if

$$
\forall(\boldsymbol{x}, \boldsymbol{y}) \in\left(\mathbb{R}^{m}\right)^{2} \quad f(\boldsymbol{x}, \boldsymbol{y})=\mathbf{0} \quad \Longleftrightarrow \quad \boldsymbol{x} \geq \mathbf{0}, \quad \boldsymbol{y} \geq \mathbf{0}, \quad \boldsymbol{x} \cdot \boldsymbol{y}=0 .
$$

Examples of $C$-functions are respectively the min, max, Fischer-Burmeister or the Mangasarian functions,

$$
\begin{aligned}
(\min \{\boldsymbol{x}, \boldsymbol{y}\})_{l}:=\min \left\{\boldsymbol{x}_{l}, \boldsymbol{y}_{l}\right\}, & l=1, \ldots, m, \\
(\max \{\boldsymbol{x}, \boldsymbol{y}\})_{l}:=\max \left\{\boldsymbol{x}_{l}, \boldsymbol{y}_{l}\right\} & l=1, \ldots, m, \\
\left(f_{\mathrm{FB}}(\boldsymbol{x}, \boldsymbol{y})\right)_{l}:=\sqrt{\boldsymbol{x}_{l}^{2}+\boldsymbol{y}_{l}^{2}}-\left(\boldsymbol{x}_{l}+\boldsymbol{y}_{l}\right) & l=1, \ldots, m, \\
\left(f_{\mathrm{M}}(\boldsymbol{x}, \boldsymbol{y})\right)_{l}:=\xi\left(\left|\boldsymbol{x}_{l}-\boldsymbol{y}_{l}\right|\right)-\xi\left(\boldsymbol{y}_{l}\right)-\xi\left(\boldsymbol{x}_{l}\right) & l=1, \ldots, m,
\end{aligned}
$$


where $\xi: \mathbb{R} \mapsto \mathbb{R}$ is an increasing function satisfying $\xi(\mathbf{0})=\mathbf{0}$. These functions take rather simple forms, but unfortunately they are not Fréchet differentiable everywhere. Let $\tilde{\mathbf{C}}$ be any $C$-function satisfying for $m=\mathcal{N}^{p, \mathrm{i}} \tilde{\mathbf{C}}\left(\boldsymbol{X}_{1 h}^{n}+g \mathbf{1}-\boldsymbol{X}_{2 h}^{n}, \boldsymbol{X}_{3 h}^{n}\right)=0 \Longleftrightarrow \boldsymbol{X}_{1 h}^{n}+g \mathbf{1}-\boldsymbol{X}_{2 h}^{n} \geq 0, \boldsymbol{X}_{3 h}^{n} \geq 0$, and $\left(\boldsymbol{X}_{1 h}^{n}+g \mathbf{1}-\boldsymbol{X}_{2 h}^{n}\right) \cdot \boldsymbol{X}_{3 h}^{n}=$ 0 . Then, introducing the function $\mathbf{C}: \mathbb{R}^{3 \mathcal{N}^{p, i}} \rightarrow \mathbb{R}^{\mathcal{N}^{p, i}}$ defined as $\mathbf{C}\left(\boldsymbol{X}_{h}^{n}\right):=\tilde{\mathbf{C}}\left(\boldsymbol{X}_{1 h}^{n}+g \mathbf{1}-\boldsymbol{X}_{2 h}^{n}, \boldsymbol{X}_{3 h}^{n}\right)$, problem (3.12) can be equivalently rewritten: for each $n \geq 1$, given $\boldsymbol{X}_{h}^{n-1} \in \mathbb{R}^{2 \mathcal{N}^{p, i}}$, search $\boldsymbol{X}_{h}^{n} \in \mathbb{R}^{3 \mathcal{N}^{p, i}}$ such that

$$
\begin{cases}\mathbb{E}_{p}^{n} \boldsymbol{X}_{h}^{n}=\boldsymbol{F}^{n} \\ \mathbf{C}\left(\boldsymbol{X}_{h}^{n}\right)=\mathbf{0}\end{cases}
$$

\subsection{Linearization by semismooth Newton methods}

Let a time step $n \geq 1$ be fixed and let $\boldsymbol{X}_{h}^{n-1} \in \mathbb{R}^{2 \mathcal{N}^{p, \mathrm{i}}}$ be given. We describe in this section the linearization of system (3.14) by a semismooth Newton method. Remark that the Newton method cannot be applied to (3.14) since $\mathbf{C}$ is not Fréchet differentiable. Observe more precisely that the first $2 \mathcal{N}^{p, i}$ lines of (3.14) are linear and the nonlinearity occurs in the last $\mathcal{N}^{p, i}$ lines of (3.14). The function $\mathbf{C}$ is locally Lipschitz and continuous so that it is differentiable almost everywhere. The semismooth Newton linearization [16, 17, 40] is defined as follows: let an initial guess $\boldsymbol{X}_{h}^{n, 0} \in \mathbb{R}^{3 \mathcal{N}^{p, i}}$ be given; typically, $\boldsymbol{X}_{h}^{n, 0}:=\boldsymbol{X}_{h}^{n-1}$, where $\boldsymbol{X}_{h}^{n-1}$ is the last iterate from the previous time step. At linearization step $k \geq 1$, one looks for $\boldsymbol{X}_{h}^{n, k} \in \mathbb{R}^{3 \mathcal{N}^{p, i}}$ such $^{2}$ that

$$
\mathbb{A}^{n, k-1} \boldsymbol{X}_{h}^{n, k}=\boldsymbol{B}^{n, k-1},
$$

where the matrix $\mathbb{A}^{n, k-1} \in \mathbb{R}^{3 \mathcal{N}^{p, \mathrm{i}}, 3 \mathcal{N}^{p, \mathrm{i}}}$ and the right-hand side vector $\boldsymbol{B}^{n, k-1} \in \mathbb{R}^{3 \mathcal{N}^{p, \mathrm{i}}}$ are respectively given by

$$
\mathbb{A}^{n, k-1}:=\left[\begin{array}{c}
\mathbb{E}_{p}^{n} \\
\mathbf{J}_{\mathbf{C}}\left(\boldsymbol{X}_{h}^{n, k-1}\right)
\end{array}\right], \boldsymbol{B}^{n, k-1}:=\left[\begin{array}{c}
\boldsymbol{F}^{n} \\
\mathbf{J}_{\mathbf{C}}\left(\boldsymbol{X}_{h}^{n, k-1}\right) \boldsymbol{X}_{h}^{n, k-1}-\mathbf{C}\left(\boldsymbol{X}_{h}^{n, k-1}\right)
\end{array}\right]
$$

Here, the notation $\mathbf{J}_{\mathbf{C}}\left(\boldsymbol{X}_{h}^{n, k-1}\right)$ stands for the Jacobian matrix in the sense of Clarke [16, 17].

For illustration, consider the semismooth min function (3.13a). Then

$$
\min \left\{\boldsymbol{X}_{1 h}^{n}+g \mathbf{1}-\boldsymbol{X}_{2 h}^{n}, \boldsymbol{X}_{3 h}^{n}\right\}=\min \left\{\left(\begin{array}{c}
u_{1 h}^{n}\left(\boldsymbol{x}_{1}\right)-u_{2 h}^{n}\left(\boldsymbol{x}_{1}\right) \\
\vdots \\
u_{1 h}^{n}\left(\boldsymbol{x}_{\mathcal{N}^{p, i}}\right)-u_{2 h}^{n}\left(\boldsymbol{x}_{\mathcal{N}^{p, i}}\right)
\end{array}\right),\left(\begin{array}{c}
\left(\boldsymbol{X}_{3 h}^{n}\right)_{1} \\
\vdots \\
\left(\boldsymbol{X}_{3 h}^{n}\right)_{\mathcal{N} p, \mathrm{i}}
\end{array}\right)\right\} .
$$

Let the block matrices $\mathbb{K}$ and $\mathbb{G}$ in $\mathbb{R}^{\mathcal{N}^{p, \mathrm{i}}, 3 \mathcal{N}^{p, \mathrm{i}}}$ be defined respectively by $\mathbb{K}:=\left[\mathbb{I}_{\mathrm{d}},-\mathbb{I}_{\mathrm{d}}, \mathbf{0}\right]$, and $\mathbb{G}:=\left[\mathbf{0}, \mathbf{0}, \mathbb{I}_{\mathrm{d}}\right]$. The $l^{\text {th }}$ row of the Jacobian matrix $\mathbf{J}_{\mathbf{C}}\left(\boldsymbol{X}_{h}^{n, k-1}\right)$ is given by the $l^{\text {th }}$ row of $\mathbb{K}$ if $u_{1 h}^{n, k-1}\left(\boldsymbol{x}_{l}\right)-u_{2 h}^{n, k-1}\left(\boldsymbol{x}_{l}\right) \leq$ $\left(\boldsymbol{X}_{3 h}^{n, k-1}\right)_{l}$, and by the $l^{\text {th }}$ row of $\mathbb{G}$ if $u_{1 h}^{n, k-1}\left(\boldsymbol{x}_{l}\right)-u_{2 h}^{n, k-1}\left(\boldsymbol{x}_{l}\right)>\left(\boldsymbol{X}_{3 h}^{n, k-1}\right)_{l}$.

\subsection{Iterative algebraic solvers}

Let a linearization step $k \geq 1$ be fixed, and apply an iterative algebraic solver with iteration index $i \geq 0$ to the linear system (3.15). Given an initial guess $\boldsymbol{X}_{h}^{n, k, 0} \in \mathbb{R}^{3 \mathcal{N}^{p, 1}}$, often taken as $\boldsymbol{X}_{h}^{n, k, 0}:=\boldsymbol{X}_{h}^{n, k-1}$, where $\boldsymbol{X}_{h}^{n, k-1}$ is the last available iterate from the previous semismooth Newton step, the algebraic residual vector on step $i$ is defined by

$$
\boldsymbol{R}_{h}^{n, k, i}:=\boldsymbol{B}^{n, k-1}-\mathbb{A}^{n, k-1} \boldsymbol{X}_{h}^{n, k, i}
$$

It is a block vector $\left[\boldsymbol{R}_{h}^{n, k, i}\right]^{T}:=\left[\boldsymbol{R}_{1 h}^{n, k, i}, \boldsymbol{R}_{2 h}^{n, k, i}, \boldsymbol{R}_{3 h}^{n, k, i}\right]^{T} \in \mathbb{R}^{3 \mathcal{N}^{p, \mathrm{i}}}$, where $\boldsymbol{R}_{\alpha h}^{n, k, i} \in \mathcal{N}^{p, \mathrm{i}}, \alpha=1,2$, are the components associated to $(3.12 \mathrm{a})$, whereas $\boldsymbol{R}_{3 h}^{n, k, i} \in \mathcal{N}^{p, \mathrm{i}}$ is a representation of the constraints $(3.12 \mathrm{~b})$. The algebraic solver can be stopped when the relative algebraic residual satisfies

$$
\left\|\boldsymbol{R}_{h}^{n, k, i}\right\| /\left\|\boldsymbol{B}^{n, k-1}-\mathbb{A}^{n, k-1} \boldsymbol{X}_{h}^{n, k, 0}\right\| \leq \varepsilon_{\text {alg }}^{k} .
$$




\subsection{Exact semismooth Newton method}

The "exact semismooth Newton" method requires to solve "exactly" the linear (3.15) system. To achieve this numerically, we consider an iterative solver applied to (3.15), stopped when the criterion (3.18) is satisfied with $\varepsilon_{\mathrm{alg}}^{k}$ close to the machine precision. For the linearization stopping criterion, we choose a tolerance $\varepsilon_{\text {lin }}$ close to the machine precision and stop the linearization procedure when the relative linearization residual satisfies

$$
\left\|\left(\begin{array}{c}
\boldsymbol{F}^{n}-\mathbb{E}_{p}^{n} \boldsymbol{X}_{h}^{n, k} \\
\mathbf{C}\left(\boldsymbol{X}_{h}^{n, k}\right)
\end{array}\right)\right\| /\left\|\left(\begin{array}{c}
\boldsymbol{F}^{n}-\mathbb{E}_{p}^{n} \boldsymbol{X}_{h}^{n, 0} \\
\mathbf{C}\left(\boldsymbol{X}_{h}^{n, 0}\right)
\end{array}\right)\right\| \leq \varepsilon_{\text {lin }},
$$

which means that $(3.14)$ is solved up to $\varepsilon_{\text {lin }}$.

\subsection{Traditional inexact semismooth Newton method}

A traditional "inexact semismooth Newton" resolution of (3.14) consists in, on each step $k \geq 1$, stopping the algebraic solver when (3.18) is satisfied with the forcing term $\varepsilon_{\text {alg }}^{k}$ that can be "large" and generally depends on $k$, see $[41,42,40]$. To stop the iterations in $k,(3.19)$ is typically employed.

\subsection{Adaptive inexact semismooth Newton method}

We provide in Section 5.2 below an alternative to the classical stopping criteria (3.18) and (3.19), giving rise to our "adaptive inexact semismooth Newton" linearization, see (5.13) and (5.14). We do not steer the stopping via the $l^{2}$-norm of the residual vectors, but via a posteriori error estimators, derived in the energy norm. We also typically stop earlier the iterations both in $i$ and $k$.

\subsection{Notations for the updates}

When the algebraic stopping criterion is satisfied, we update the solution

$$
\boldsymbol{X}_{h}^{n, k}:=\boldsymbol{X}_{h}^{n, k, i} .
$$

Once the linearization stopping criterion is met, we update the solution

$$
\boldsymbol{X}_{h}^{n}:=\boldsymbol{X}_{h}^{n, k}
$$

Thus, $u_{1 h}^{n-1}, u_{2 h}^{n-1}$, and $\lambda_{h}^{n-1}$ are the functional representations of the vectors $\boldsymbol{X}_{1 h}^{n-1}, \boldsymbol{X}_{2 h}^{n-1}$, and $\boldsymbol{X}_{3 h}^{n-1}$, i.e. $\boldsymbol{X}_{\alpha h}^{n-1, k, i}$ when the stopping criteria are met.

\section{A posteriori error analysis}

In this section, we derive two a posteriori error estimates. First, we establish an a posteriori error estimate when $p=1$ and when both the algebraic and linearization solvers have converged. Next, we derive an a posteriori error estimate when $p \geq 1$ at any semismooth linearization step $k \geq 1$ and any step of the iterative algebraic solver $i \geq 0$.

Our a posteriori analysis relies on the equilibrated flux reconstructions following the concepts of [43, 44]. A discretization flux reconstruction $\boldsymbol{\sigma}_{\alpha h, \mathrm{disc}}^{n, k, i} \in \mathbf{H}(\operatorname{div}, \Omega)$, and an algebraic error flux reconstruction $\boldsymbol{\sigma}_{\alpha h, \text { alg }}^{n, k, i} \in \mathbf{H}(\operatorname{div}, \Omega)$ following the methodology of [45, Concept 4.1] are constructed. The sum of these two fluxes represents a consistent (in $\mathbf{H}(\operatorname{div}, \Omega)$ ) reconstruction of the gradient of $\boldsymbol{u}_{h}^{n}$ (up to the sign and constants $\mu_{1}$ and $\mu_{2}$ ). These reconstruction are devised to capture components of error linked respectively to discretization or to the algebraic resolution. Note that as the first equation in (3.14) is linear, there is no need for an additional linearization flux reconstruction as in [46]. We present very briefly the developments here. Details can be found in [38, Sec. 2.6]. 


\subsection{Approximate solution}

At each time step $1 \leq n \leq N_{\mathrm{t}}$, the approximation to the nonlinear system (3.14) gives the $\boldsymbol{X}_{h}^{n, k, i} \in \mathbb{R}^{3 \mathcal{N}^{p, i}}$, where $k \geq 1$ is the semismooth Newton step and $i \geq 0$ is the algebraic solver step. The functional representations of the vectors $\boldsymbol{X}_{1 h}^{n, k, i}, \boldsymbol{X}_{2 h}^{n, k, i}$, and $\boldsymbol{X}_{3 h}^{n, k}, i$ are then $u_{1 h}^{n, k, i}, u_{2 h}^{n, k, i}$ and $\lambda_{h}^{n, k, i}$, as in (3.11). Obviously, $\left(u_{1 h}^{n, k, i}, u_{2 h}^{n, k, i}, \lambda_{h}^{n, k, i}\right) \in X_{g h}^{p} \times X_{0 h}^{p} \times X_{h}^{p}, \forall 1 \leq n \leq N_{\mathrm{t}}$. We associate to the space functions $u_{1 h}^{n, k, i}$ and $u_{2 h}^{n, k, i}$, their space-time representations $u_{1 h \tau}^{k, i}, u_{2 h \tau}^{k, i}$

$$
\begin{array}{ll}
\left.u_{1 h \tau}^{k, i}\right|_{I_{n}}:=\frac{u_{1 h}^{n, k, i}-u_{1 h}^{n-1}}{\Delta t_{n}}\left(t-t^{n}\right)+u_{1 h}^{n, k, i} & \forall 1 \leq n \leq N_{\mathrm{t}}, \\
\left.u_{2 h \tau}^{k, i}\right|_{I_{n}}:=\frac{u_{2 h}^{n, k, i}-u_{2 h}^{n-1}}{\Delta t_{n}}\left(t-t^{n}\right)+u_{2 h}^{n, k, i} \quad & \forall 1 \leq n \leq N_{\mathrm{t}} .
\end{array}
$$

Concerning the discrete Lagrange multiplier $\lambda_{h}^{n, k, i} \in X_{h}^{p}$, its space-time representation is defined by a piecewise constant-in-time function $\lambda_{h \tau}^{k, i}$

$$
\left.\lambda_{h \tau}^{k, i}\right|_{I_{n}}:=\lambda_{h}^{n, k, i}
$$

Note that this construction ensures that $u_{\alpha h \tau}^{k, i}, \alpha=1,2$, are continuous and piecewise affine in time, so that $\partial_{t} u_{\alpha h \tau}^{k, i} \in L^{2}\left(0, T ; H^{-1}(\Omega)\right)$. In the expressions of $u_{1 h \tau}^{k, i}, u_{2 h \tau}^{k, i}$, and $\lambda_{h \tau}^{k, i}$, the indices $k, i$ are kept to indicate the presence of inexact solvers; more precisely, $u_{\alpha h}^{n-1}$ are equal to $u_{\alpha h}^{n-1, k, i}$ for the last iterates $k$ and $i$ on time step $n-1$ when the stopping criteria are met, see Section 3.10. For each time step $n$, we also denote

$$
u_{1 h \tau}^{n, k, i}:=\left.u_{1 h \tau}^{k, i}\right|_{I_{n}}, \quad u_{2 h \tau}^{n, k, i}:=\left.u_{2 h \tau}^{k, i}\right|_{I_{n}},
$$

so that

$$
\left.\partial_{t} u_{1 h \tau}^{n, k, i}\right|_{I_{n}}=\frac{1}{\Delta t_{n}}\left(u_{1 h}^{n, k, i}-u_{1 h}^{n-1}\right),\left.\quad \partial_{t} u_{2 h \tau}^{n, k, i}\right|_{I_{n}}=\frac{1}{\Delta t_{n}}\left(u_{2 h}^{n, k, i}-u_{2 h}^{n-1}\right)
$$

\subsection{Representation of the residual}

To proceed, we need to give a functional representation to (3.17). Following [45], we associate respectively with $\boldsymbol{R}_{1 h}^{n, k, i}$ and $\boldsymbol{R}_{2 h}^{n, k, i}$ discontinuous elementwise polynomials $r_{1 h}^{n, k, i}$ and $r_{2 h}^{n, k, i}$ of degree $p \geq 1$ that vanish on the boundary of $\Omega$. These can be easily computed solving on each element $K \in \mathcal{T}_{h}$ a small problem with an element mass matrix given as follows. For $\boldsymbol{x}_{l} \in \mathcal{V}^{p, \mathrm{i}}$, denote by $N_{h, \boldsymbol{x}_{l}}$ the number of mesh elements forming the support of the basis function $\psi_{h, \boldsymbol{x}_{l}}$. Then, for all $K \in \mathcal{T}_{h}$ and for all $\alpha \in\{1,2\}$, define $\left.r_{\alpha h}^{n, k, i}\right|_{K} \in \mathbb{P}_{p}(K)$ such that:

$$
\left(r_{\alpha h}^{n, k, i}, \psi_{h, \boldsymbol{x}_{l}}\right)_{K}:=\frac{\left(\boldsymbol{R}_{\alpha h}^{n, k, i}\right)_{l}}{N_{h, \boldsymbol{x}_{l}}} \quad \text { and }\left.\quad r_{\alpha h}^{k, i}\right|_{\partial K \cap \partial \Omega}:=0
$$

for all Lagrange basis functions $\psi_{h, \boldsymbol{x}_{l}} \in X_{h}^{p}, \boldsymbol{x}_{l} \in \mathcal{V}^{p, \mathrm{i}}$, nonzero on $K$. It is easily seen that the first $2 \mathcal{N}^{p, \mathrm{i}}$ lines of (3.17) then read: $\forall l=1, \ldots, \mathcal{N}^{p, \mathrm{i}}$,

$$
\begin{aligned}
& \mu_{1}\left(\nabla u_{1 h}^{n, k, i}, \nabla \psi_{h, \boldsymbol{x}_{l}}\right)_{\Omega}=\left(\tilde{f}_{1}^{n}+\tilde{\lambda}_{h, l}^{n, k, i}-r_{1 h}^{n, k, i}-\partial_{t} u_{1 h \tau}^{n, k, i}, \psi_{h, \boldsymbol{x}_{l}}\right)_{\Omega}, \\
& \mu_{2}\left(\nabla u_{2 h}^{n, k, i}, \nabla \psi_{h, \boldsymbol{x}_{l}}\right)_{\Omega}=\left(\tilde{f}_{2}^{n}-\tilde{\lambda}_{h, l}^{n, k, i}-r_{2 h}^{n, k, i}-\partial_{t} u_{2 h \tau}^{n, k, i}, \psi_{h, \boldsymbol{x}_{l}}\right)_{\Omega},
\end{aligned}
$$

where

$$
\tilde{\lambda}_{h, l}^{n, k, i}:=\left\{\begin{array}{l}
\left.\lambda_{h}^{n, k, i}\left(\boldsymbol{x}_{l}\right) \text { (real number given by the vertex value of } \lambda_{h}^{k, i}\right) \text { if } p=1, \\
\lambda_{h}^{n, k, i} \text { (function } \lambda_{h}^{n, k, i}, \text { the index } l \text { is discarded) if } p \geq 2 .
\end{array}\right.
$$

We also use the shorthand notation for $\boldsymbol{a} \in \mathcal{V}_{h}$

$$
\tilde{\lambda}_{h, \boldsymbol{a}}^{n, k, i}= \begin{cases}\lambda_{h}^{n, k, i}(\boldsymbol{a}) & \text { if } \quad p=1 \\ \lambda_{h}^{n, k, i} & \text { if } \quad p \geq 2\end{cases}
$$




\subsection{Flux reconstructions}

Let $\alpha \in\{1,2\}, 1 \leq n \leq N_{\mathrm{t}}, k \geq 1$, and $i \geq 0$ be fixed. The discretization fluxes are constructed by solving local mixed problems in patches of elements $\omega_{h}^{\boldsymbol{a}}$ for each vertex $\boldsymbol{a} \in \mathcal{V}_{h}$. This provides $\boldsymbol{\sigma}_{\alpha h, \text { disc }}^{n, k, i, \boldsymbol{a}}$, and their sums $\boldsymbol{\sigma}_{\alpha h, \text { disc }}^{n, k, i}:=\sum_{\boldsymbol{a} \in \mathcal{V}_{h}} \boldsymbol{\sigma}_{\alpha h, \text { disc }}^{n, k, i, \boldsymbol{a}}$ are the discretization fluxes. With respect to the stationary case [28, Sec. 4.2], the only difference is the source term for the divergence equation in the mixed system:

$$
\tilde{g}_{\alpha h}^{n, k, i, \boldsymbol{a}}:=\left(\tilde{f}_{\alpha}^{n}-(-1)^{\alpha} \tilde{\lambda}_{h, \boldsymbol{a}}^{n, k, i}-r_{\alpha h}^{n, k, i}-\left.\partial_{t} u_{\alpha h \tau}^{n, k, i}\right|_{\omega_{h}^{a}}\right) \psi_{h, \boldsymbol{a}}-\mu_{\alpha} \nabla u_{\alpha h}^{n, k, i} \cdot \nabla \psi_{h, \boldsymbol{a}},
$$

where $\tilde{\lambda}_{h, \boldsymbol{a}}^{n, k, i}$ and $r_{\alpha h}^{n, k, i}$ are defined in the previous section. This yields $\boldsymbol{\sigma}_{\alpha h, \mathrm{disc}}^{n, k, i} \in \mathbf{R T}_{p}(\Omega) \subset \mathbf{H}(\operatorname{div}, \Omega)$, where $\mathbf{R T}_{p}(\Omega)$ is the Raviart-Thomas subspace of $\mathbf{H}(\operatorname{div}, \Omega)$ of order $p \geq 1$, [47].

The algebraic error flux reconstructions $\boldsymbol{\sigma}_{\alpha h, \mathrm{alg}}^{n, k, i}$ are obtained by the methodology of [45, Concept 4.1] and yield

$$
\boldsymbol{\sigma}_{\alpha h, \mathrm{alg}}^{n, k, i} \in \mathbf{R T}_{p}(\Omega) \subset \mathbf{H}(\operatorname{div}, \Omega) \quad \text { and } \quad \boldsymbol{\nabla} \cdot \boldsymbol{\sigma}_{\alpha h, \mathrm{alg}}^{n, k, i}=r_{\alpha h}^{n, k, i} .
$$

The total flux reconstructions are the sums

$$
\boldsymbol{\sigma}_{\alpha h}^{n, k, i}:=\boldsymbol{\sigma}_{\alpha h, \mathrm{disc}}^{n, k, i}+\boldsymbol{\sigma}_{\alpha h, \mathrm{alg}}^{n, k, i} \quad \alpha=1,2 .
$$

They crucially satisfy

$$
\left(\boldsymbol{\nabla} \cdot \boldsymbol{\sigma}_{\alpha h}^{n, k, i}, q_{h}\right)_{K}=\left(\tilde{f}_{\alpha}^{n}-(-1)^{\alpha} \lambda_{h}^{n, k, i}-\partial_{t} u_{\alpha h \tau}^{n, k, i}, q_{h}\right)_{K} \quad \forall q_{h} \in \mathbb{P}_{p}(K), \forall K \in \mathcal{T}_{h}
$$

For $\alpha=1,2$, we finally define the piecewise-constant-in-time reconstructions,

$$
\begin{aligned}
& \left(\boldsymbol{\sigma}_{\alpha h \tau}^{k, i}, \boldsymbol{\sigma}_{\alpha h \tau, \mathrm{disc}}^{k, i}, \boldsymbol{\sigma}_{\alpha h \tau, \mathrm{alg}}^{k, i}\right) \in\left[L^{2}(0, T ; \mathbf{H}(\operatorname{div}, \Omega))\right]^{3}, \\
& \left.\boldsymbol{\sigma}_{\alpha h \tau}^{k, i}\right|_{I_{n}}=\boldsymbol{\sigma}_{\alpha h}^{n, k, i},\left.\quad \boldsymbol{\sigma}_{\alpha h \tau, \operatorname{disc}}^{k, i}\right|_{I_{n}}=\boldsymbol{\sigma}_{\alpha h, \mathrm{disc}}^{n, k, i},\left.\quad \boldsymbol{\sigma}_{\alpha h \tau, \mathrm{alg}}^{k, i}\right|_{I_{n}}=\boldsymbol{\sigma}_{\alpha h, \mathrm{alg}}^{n, k, i}, \quad \forall 1 \leq n \leq N_{\mathrm{t}} .
\end{aligned}
$$

\subsection{An a posteriori error estimate for $p=1$ and exact solvers}

In this section, we establish an a posteriori error estimate between the exact solution $\boldsymbol{u} \in \mathcal{K}_{g}^{\mathrm{t}}$ given by (1.2) and the approximate numerical solution $\boldsymbol{u}_{h \tau}$, for $p=1$ when the semismooth Newton solver and the iterative algebraic solver have converged. Here, we discard the indices $k$ and $i$. Recall that when $p=1$ and for exact solvers, the constraints in (3.9) imply that the approximate solution is conforming in the sense that $\boldsymbol{u}_{h \tau} \in \mathcal{K}_{g}^{\mathrm{t}}$ and $\lambda_{h \tau} \in \Psi$.

Definition 4.1. Let $1 \leq n \leq N_{\mathrm{t}}, K \in \mathcal{T}_{h}$, and $\alpha=1,2$. We define the residual estimator $\eta_{\mathrm{R}, K, \alpha}^{n}$, the flux estimator $\eta_{\mathrm{F}, K, \alpha}^{n}$, the constraint estimator $\eta_{\mathrm{C}, K}^{n}$, and the data oscillation estimator $\eta_{\mathrm{osc}, K, \alpha}^{n}$ by the temporal functions, for all $t \in I_{n}$,

$$
\begin{aligned}
\eta_{\mathrm{R}, K, \alpha}^{n}(t) & :=\frac{h_{K}}{\pi} \mu_{\alpha}^{-\frac{1}{2}}\left\|\tilde{f}_{\alpha}^{n}-\partial_{t} u_{\alpha h \tau}^{n}-(-1)^{\alpha} \lambda_{h}^{n}-\nabla \cdot \sigma_{\alpha h}^{n}\right\|_{K}, \\
\eta_{\mathrm{F}, K, \alpha}^{n}(t) & :=\left\|\mu_{\alpha}^{\frac{1}{2}} \nabla u_{\alpha h \tau}^{n}+\mu_{\alpha}^{-\frac{1}{2}} \sigma_{\alpha h}^{n}\right\|_{K}, \\
\eta_{\mathrm{C}, K}^{n}(t) & :=2\left(\lambda_{h}^{n}, u_{1 h \tau}^{n}-u_{2 h \tau}^{n}\right)_{K}, \\
\eta_{\mathrm{osc}, K, \alpha}^{n}(t) & :=C_{\mathrm{PF}} h_{\Omega} \mu_{\alpha}^{-\frac{1}{2}}\left\|f_{\alpha}-\tilde{f}_{\alpha}^{n}\right\|_{K} .
\end{aligned}
$$

Remark 4.2. The estimators (4.7)-(4.10) are an extension of the estimators of [28] derived in the case of elliptic variational inequations to the parabolic case. They reflect various violations of physical properties of the approximate solution $\left(u_{1 h \tau}^{n}, u_{2 h \tau}^{n}, \lambda_{h \tau}^{n}\right): \eta_{\mathrm{R}, K, \alpha}^{n}$ and $\eta_{\mathrm{F}, K, \alpha}^{n}$ represent the nonconformity of the flux, i.e., the fact that $-\mu_{\alpha} \nabla u_{\alpha h \tau}^{n} \notin L^{2}(0, T ; \mathbf{H}(\operatorname{div}, \Omega)) ; \eta_{\mathrm{C}, K}^{n}$ reflects inconsistencies in the complementarity conditions at the discrete level, i.e., the fact that $\left(u_{1 h \tau}^{n}-u_{2 h \tau}^{n}\right) \lambda_{h}^{n} \neq 0$. Note that the last constraint in (3.9) for $p=1$ only requires that $\left(u_{1 h}^{n}-u_{2 h}^{n}\right) \lambda_{h}^{n}$ vanishes at each vertex of $\mathcal{T}_{h}$ but not everywhere in $\Omega$ and not on the whole time interval $I_{n}$. Finally, $\eta_{\mathrm{osc}, K, \alpha}^{n}$ represents the local distance between the right hand side and its time-averages over $I_{n}$. Note that this latter term is an estimator of $\left\|f_{\alpha}-\tilde{f}_{\alpha}^{n}\right\|_{H^{-1}(\Omega)}$ (see (4.16) further) with a rather pessimistic constant, see the discussion in [31, Rem. 5.4] and the references therein). 


\subsubsection{A control of the energy error}

Recall the Poincaré-Friedrichs and the Poincaré-Wirtinger inequalities, denoting by $\bar{v}_{\mathcal{O}}$ the mean value of $v$ over domain $\mathcal{O}$ and $h_{\mathcal{O}}$ the diameter of $\mathcal{O}$,

$$
\begin{array}{rlrl}
\|v\|_{\mathcal{O}} & \leq C_{\mathrm{PF}} h_{\mathcal{O}}\|\nabla v\|_{\mathcal{O}} & \forall v \in H_{0}^{1}(\mathcal{O}) \\
\left\|v-\bar{v}_{\mathcal{O}}\right\|_{\mathcal{O}} \leq C_{\mathrm{PW}} h_{\mathcal{O}}\|\nabla v\|_{\mathcal{O}} & \forall v \in H^{1}(\mathcal{O}) .
\end{array}
$$

We then have:

Theorem 4.3 (case $p=1$ and exact solvers). Let $\boldsymbol{u} \in \mathcal{K}_{g}^{\mathrm{t}}$ be the exact solution given by (1.2). Let $\boldsymbol{u}_{h \tau} \in \mathcal{K}_{g}^{\mathrm{t}}$ and $\lambda_{h \tau} \in \Psi$ be the approximate solutions for $p=1$ and exact solvers. Consider the equilibrated flux reconstructions $\boldsymbol{\sigma}_{\alpha h \tau} \in L^{2}(0, T ; \mathbf{H}(\operatorname{div}, \Omega))$ given by (4.4), (4.6). Using the error estimators defined by (4.7)-(4.10), there holds

$$
\begin{aligned}
& \left\|\boldsymbol{u}-\boldsymbol{u}_{h \tau}\right\|_{\Omega, T}^{2}+\left\|\left(\boldsymbol{u}-\boldsymbol{u}_{h \tau}\right)(\cdot, T)\right\|_{\Omega}^{2} \leq \eta^{2}:= \\
& \left\{\left(\sum_{n=1}^{N_{\mathrm{t}}} \int_{I_{n}} \sum_{\alpha=1}^{2} \sum_{K \in \mathcal{T}_{h}}\left(\eta_{\mathrm{R}, K, \alpha}^{n}+\eta_{\mathrm{F}, K, \alpha}^{n}\right)^{2}\right)^{\frac{1}{2}}+\left(\sum_{n=1}^{N_{\mathrm{t}}} \int_{I_{n}} \sum_{\alpha=1}^{2} \sum_{K \in \mathcal{T}_{h}}\left(\eta_{\mathrm{osc}, K, \alpha}^{n}\right)^{2}\right)^{\frac{1}{2}}\right\}^{2} \\
& +\sum_{n=1}^{N_{\mathrm{t}}} \int_{I_{n}} \sum_{K \in \mathcal{T}_{h}} \eta_{\mathrm{C}, K}^{n}(t) \mathrm{dt}+\left\|\left(\boldsymbol{u}-\boldsymbol{u}_{h \tau}\right)(\cdot, 0)\right\|_{\Omega}^{2} .
\end{aligned}
$$

To prove Theorem 4.3, we first introduce the following lemma.

Lemma 4.4. Let $a$ and $b$ be the forms defined in (2.3). Let $\boldsymbol{u} \in \mathcal{K}_{g}^{\mathrm{t}}$ be the weak solution from (1.2) and let $\boldsymbol{y}:=\left(y_{1}, y_{2}\right) \in \mathcal{K}_{g}^{\mathrm{t}}$ be arbitrary. Then, for the vector $\boldsymbol{y}^{*}:=\left(y_{1}^{*}, y_{2}^{*}\right):=\left(u_{1}-y_{1}, u_{2}-y_{2}\right) \in$ $\left[L^{2}\left(0, T ; H_{0}^{1}(\Omega)\right)\right]^{2}$, there holds

$$
\begin{aligned}
A & :=\int_{0}^{T}\left(\left(\boldsymbol{f}, \boldsymbol{y}^{*}\right)_{\Omega}-\left(\partial_{t} \boldsymbol{u}_{h \tau}, \boldsymbol{y}^{*}\right)_{\Omega}-a\left(\boldsymbol{u}_{h \tau}, \boldsymbol{y}^{*}\right)+b\left(\boldsymbol{y}^{*}, \lambda_{h \tau}\right)\right)(t) \mathrm{dt} \\
& \leq\left(\left\{\sum_{n=1}^{N_{\mathrm{t}}} \int_{I_{n}} \sum_{\alpha=1}^{2} \sum_{K \in \mathcal{T}_{h}}\left(\eta_{\mathrm{R}, K, \alpha}^{n}+\eta_{\mathrm{F}, K, \alpha}^{n}\right)^{2}(t) \mathrm{dt}\right\}^{\frac{1}{2}}+\left\{\sum_{n=1}^{N_{\mathrm{t}}} \int_{I_{n}} \sum_{\alpha=1}^{2} \sum_{K \in \mathcal{T}_{h}}\left(\eta_{\mathrm{osc}, K, \alpha}^{n}\right)^{2}(t) \mathrm{dt}\right\}^{\frac{1}{2}}\right)\left\|\boldsymbol{y}^{*}\right\|_{\Omega, T} .
\end{aligned}
$$

Proof. Adding and subtracting $\boldsymbol{\sigma}_{\alpha h \tau}(t) \in \mathbf{H}(\operatorname{div}, \Omega)$, using the Green formula with $y_{\alpha}^{*}(t) \in H_{0}^{1}(\Omega), \alpha=1,2$, and employing the decomposition $f_{\alpha}=\tilde{f}_{\alpha}^{n}+\left(f_{\alpha}-\tilde{f}_{\alpha}^{n}\right)$, we have

$$
\begin{aligned}
A= & \int_{0}^{T} \sum_{\alpha=1}^{2}\left(\tilde{f}_{\alpha}^{n}-\partial_{t} u_{\alpha h \tau}-\nabla \cdot \boldsymbol{\sigma}_{\alpha h \tau}-(-1)^{\alpha} \lambda_{h \tau}, y_{\alpha}^{*}\right)_{\Omega}(t) \mathrm{dt} \\
& -\int_{0}^{T} \sum_{\alpha=1}^{2}\left(\mu_{\alpha}^{\frac{1}{2}} \nabla u_{\alpha h \tau}+\mu_{\alpha}^{-\frac{1}{2}} \boldsymbol{\sigma}_{\alpha h \tau}, \mu_{\alpha}^{\frac{1}{2}} \nabla y_{\alpha}^{*}\right)_{\Omega}(t) \mathrm{dt}+\int_{0}^{T} \sum_{\alpha=1}^{2}\left(f_{\alpha}-\tilde{f}_{\alpha}^{n}, y_{\alpha}^{*}\right)_{\Omega}(t) \mathrm{dt} .
\end{aligned}
$$

Let $\alpha=1,2,1 \leq n \leq N_{\mathrm{t}}, t \in I_{n}$, and $K \in \mathcal{T}_{h}$ be fixed. Denoting by $\bar{w}_{K}$ the mean value over $K$ of $w \in L^{2}(\Omega)$ and using the property (4.5), one has

$$
\begin{aligned}
& \left(\tilde{f}_{\alpha}^{n}-\partial_{t} u_{\alpha h \tau}^{n}-(-1)^{\alpha} \lambda_{h}^{n}-\nabla \cdot \boldsymbol{\sigma}_{\alpha h}^{n}, y_{\alpha}^{*}\right)_{K}(t) \\
= & \left(\mu_{\alpha}^{-\frac{1}{2}}\left(\tilde{f}_{\alpha}^{n}-\partial_{t} u_{\alpha h \tau}^{n}-(-1)^{\alpha} \lambda_{h}^{n}-\nabla \cdot \boldsymbol{\sigma}_{\alpha h}^{n}\right), \mu_{\alpha}^{\frac{1}{2}}\left(y_{\alpha}^{*}-\left(\overline{y_{\alpha}^{*}}\right)_{K}\right)\right)_{K}(t) .
\end{aligned}
$$

Using the Cauchy-Schwarz inequality and next the Poincaré-Wirtinger inequality $(4.11 \mathrm{~b})$ with $C_{\mathrm{PW}}=\frac{1}{\pi}$ for the convex mesh element $K$, we get

$$
\left(\tilde{f}_{\alpha}^{n}-\partial_{t} u_{\alpha h \tau}^{n}-(-1)^{\alpha} \lambda_{h}^{n}-\nabla \cdot \boldsymbol{\sigma}_{\alpha h}^{n}, y_{\alpha}^{*}\right)_{K}(t) \leq \eta_{\mathrm{R}, K, \alpha}^{n}\left\|\mu_{\alpha}^{\frac{1}{2}} \nabla y_{\alpha}^{*}\right\|_{K}(t) .
$$


Next, as a result of the Cauchy-Schwarz inequality, we have

$$
\left(\mu_{\alpha}^{\frac{1}{2}} \nabla u_{\alpha h \tau}^{n}+\mu_{\alpha}^{-\frac{1}{2}} \boldsymbol{\sigma}_{\alpha h}^{n}, \mu_{\alpha}^{\frac{1}{2}} \nabla y_{\alpha}^{*}\right)_{K}(t) \leq \eta_{\mathrm{F}, K, \alpha}^{n}\left\|\mu_{\alpha}^{\frac{1}{2}} \nabla y_{\alpha}^{*}\right\|_{K}(t) .
$$

Finally, the Cauchy-Schwarz inequality and the Poincaré-Friedrichs inequality over the entire computational domain $\Omega$ give

$$
\begin{aligned}
\left(f_{\alpha}-\tilde{f}_{\alpha}^{n}, y_{\alpha}^{*}\right)_{\Omega}(t) & \leq C_{\mathrm{PF}} h_{\Omega} \mu_{\alpha}^{-\frac{1}{2}}\left\|f_{\alpha}-\tilde{f}_{\alpha}^{n}\right\|_{\Omega}\left\|\mu_{\alpha}^{\frac{1}{2}} \nabla y_{\alpha}^{*}\right\|_{\Omega}(t) \\
& =\left(\sum_{K \in \mathcal{T}_{h}}\left(\eta_{\mathrm{osc}, K, \alpha}^{n}\right)^{2}(t)\right)^{\frac{1}{2}}\left\|\mu_{\alpha}^{\frac{1}{2}} \nabla y_{\alpha}^{*}\right\|_{\Omega}(t) .
\end{aligned}
$$

Therefore, combining (4.14)-(4.16) and applying the Cauchy-Schwarz inequality, we get the desired result.

Proof of Theorem 4.3. From the identity

$$
\frac{1}{2}\left\|\left(\boldsymbol{u}-\boldsymbol{u}_{h \tau}\right)(\cdot, T)\right\|_{\Omega}^{2}=\frac{1}{2}\left\|\left(\boldsymbol{u}-\boldsymbol{u}_{h \tau}\right)(\cdot, 0)\right\|_{2}^{2}+\int_{0}^{T} \sum_{\alpha=1}^{2}\left\langle\partial_{t}\left(u_{\alpha}-u_{\alpha h \tau}\right), u_{\alpha}-u_{\alpha h \tau}\right\rangle(t) \mathrm{dt},
$$

posing $B:=\left\|\boldsymbol{u}-\boldsymbol{u}_{h \tau}\right\|_{\Omega, T}^{2}+\frac{1}{2}\left\|\left(\boldsymbol{u}-\boldsymbol{u}_{h \tau}\right)(\cdot, T)\right\|_{\Omega}^{2}$, using definition (2.4) and (4.17), we get

$$
B=\int_{0}^{T}\left(a\left(\boldsymbol{u}-\boldsymbol{u}_{h \tau}, \boldsymbol{u}-\boldsymbol{u}_{h \tau}\right)+\left\langle\partial_{t} \boldsymbol{u}, \boldsymbol{u}-\boldsymbol{u}_{h \tau}\right\rangle-\left(\partial_{t} \boldsymbol{u}_{h \tau}, \boldsymbol{u}-\boldsymbol{u}_{h \tau}\right)_{\Omega}\right)(t) \mathrm{dt}+\frac{1}{2}\left\|\left(\boldsymbol{u}-\boldsymbol{u}_{h \tau}\right)(\cdot, 0)\right\|_{\Omega}^{2} .
$$

Then, using the weak formulation (1.2) with $\boldsymbol{v}=\boldsymbol{u}_{h \tau} \in \mathcal{K}_{g}^{\mathrm{t}}$, we obtain

$$
B \leq \int_{0}^{T}\left(\left(\boldsymbol{f}-\partial_{t} \boldsymbol{u}_{h \tau}, \boldsymbol{u}-\boldsymbol{u}_{h \tau}\right)_{\Omega}-a\left(\boldsymbol{u}_{h \tau}, \boldsymbol{u}-\boldsymbol{u}_{h \tau}\right)\right)(t) \mathrm{dt}+\frac{1}{2}\left\|\left(\boldsymbol{u}-\boldsymbol{u}_{h \tau}\right)(\cdot, 0)\right\|_{\Omega}^{2} .
$$

Next, adding and subtracting $\int_{0}^{T} b\left(\boldsymbol{u}-\boldsymbol{u}_{h \tau}, \lambda_{h \tau}\right)(t) \mathrm{dt}$ and noting that $\left(-\lambda_{h \tau}, u_{1}-u_{2}\right)_{\Omega}(t) \leq 0$ for a.e $t \in] 0, T\left[\right.$ because $\lambda_{h \tau} \in \Psi$, we obtain

$$
\begin{aligned}
B \leq & \int_{0}^{T}\left(\left(\boldsymbol{f}-\partial_{t} \boldsymbol{u}_{h \tau}, \boldsymbol{u}-\boldsymbol{u}_{h \tau}\right)_{\Omega}-a\left(\boldsymbol{u}_{h \tau}, \boldsymbol{u}-\boldsymbol{u}_{h \tau}\right)+b\left(\boldsymbol{u}-\boldsymbol{u}_{h \tau}, \lambda_{h \tau}\right)\right)(t) \mathrm{dt} \\
& +\sum_{n=1}^{N_{t}} \int_{I_{n}} \sum_{K \in \mathcal{T}_{h}} \frac{\eta_{\mathrm{C}, K}^{n}}{2}(t) \mathrm{dt}+\frac{1}{2}\left\|\left(\boldsymbol{u}-\boldsymbol{u}_{h \tau}\right)(\cdot, 0)\right\|_{\Omega}^{2} .
\end{aligned}
$$

Finally, employing Lemma 4.4 with $\boldsymbol{y}=\boldsymbol{u}_{h \tau} \in \mathcal{K}_{g}^{\mathrm{t}}$ and using the Young inequality $A_{1} A_{2} \leq \frac{1}{2}\left(A_{1}^{2}+A_{2}^{2}\right)$, $A_{1}, A_{2} \geq 0$, we get the desired result.

\subsubsection{A control of the temporal derivative error}

So far, we have established an a posteriori error estimate between the exact solution $\boldsymbol{u} \in \mathcal{K}_{g}^{\mathrm{t}}$ and its approximate solution $\boldsymbol{u}_{h \tau} \in \mathcal{K}_{g}^{\mathrm{t}}$ in the energy norm. As we mentioned in the introduction, we cannot easily estimate the norm $\left\|\partial_{t}\left(\boldsymbol{u}-\boldsymbol{u}_{h \tau}\right)\right\|_{\left[L^{2}\left(0, T ; H^{-1}(\Omega)\right)\right]^{2}}$. We now give our replacement result. Given $\boldsymbol{u} \in \mathcal{K}_{g}^{\mathrm{t}}$ and for the approximate solution $\boldsymbol{u}_{h \tau} \in \mathcal{K}_{g}^{\mathrm{t}}$, let $\boldsymbol{z} \in \mathcal{K}_{g}^{\mathrm{t}}$ be such that, for all $\boldsymbol{v} \in \mathcal{K}_{g}^{\mathrm{t}}$,

$$
\begin{aligned}
& \int_{0}^{T} a(\boldsymbol{z}-\boldsymbol{u}, \boldsymbol{v}-\boldsymbol{z})(t) \mathrm{dt} \geq-\int_{0}^{T} \sum_{\alpha=1}^{2}\left\langle\partial_{t}\left(u_{\alpha}-u_{\alpha h \tau}\right)-(-1)^{\alpha} \lambda_{h \tau}, v_{\alpha}-z_{\alpha}\right\rangle(t) \mathrm{dt}, \\
& \boldsymbol{z}(0)=\boldsymbol{u}_{h \tau}(0) \in \mathcal{K}_{g} .
\end{aligned}
$$

As a result of the Lions-Stampacchia theorem [39], problem (4.18) is well posed. Now, we give an a posteriori error estimate on the error $\|\boldsymbol{u}-\boldsymbol{z}\|_{\Omega, T}$. 
Theorem 4.5 (case $p=1$ and exact solvers). Let $\boldsymbol{u} \in \mathcal{K}_{g}^{\mathrm{t}}$ be the solution of the weak formulation given by (1.2) and let $\boldsymbol{z} \in \mathcal{K}_{g}^{\mathrm{t}}$ be the solution of (4.18). Assume that the hypotheses of Theorem 4.3 hold and let the total estimator $\eta$ be defined by (4.12). Then

$$
\|\boldsymbol{u}-\boldsymbol{z}\|_{\Omega, T} \leq 2 \eta
$$

Proof. Setting $\boldsymbol{w}^{*}:=\boldsymbol{u}-\boldsymbol{z}$, we have $\left\|\boldsymbol{w}^{*}\right\|_{\Omega, T}^{2}=\int_{0}^{T} a(\boldsymbol{u}-\boldsymbol{z}, \boldsymbol{u}-\boldsymbol{z}) \mathrm{dt}$. For $\boldsymbol{v}=\boldsymbol{u} \in \mathcal{K}_{g}^{\mathrm{t}}$, we in turn get from (4.18)

$$
\left\|\boldsymbol{w}^{*}\right\|_{\Omega, T}^{2} \leq \int_{0}^{T}\left(\left\langle\partial_{t}\left(\boldsymbol{u}-\boldsymbol{u}_{h \tau}\right), \boldsymbol{w}^{*}\right\rangle+b\left(\boldsymbol{w}^{*}, \lambda_{h \tau}\right)\right)(t) \mathrm{dt}+\int_{0}^{T}\left(a\left(\boldsymbol{u}-\boldsymbol{u}_{h \tau}, \boldsymbol{w}^{*}\right)-a\left(\boldsymbol{u}-\boldsymbol{u}_{h \tau}, \boldsymbol{w}^{*}\right)\right)(t) \mathrm{dt}
$$

Employing the weak formulation (1.2) with $\boldsymbol{v}=\boldsymbol{z} \in \mathcal{K}_{g}^{\mathrm{t}}$ we obtain

$$
\left\|\boldsymbol{w}^{*}\right\|_{\Omega, T}^{2} \leq \int_{0}^{T}\left[\left(\boldsymbol{f}-\partial_{t} \boldsymbol{u}_{h \tau}, \boldsymbol{w}^{*}\right)_{\Omega}+b\left(\boldsymbol{w}^{*}, \lambda_{h \tau}\right)-a\left(\boldsymbol{u}_{h \tau}, \boldsymbol{w}^{*}\right)-a\left(\boldsymbol{u}-\boldsymbol{u}_{h \tau}, \boldsymbol{w}^{*}\right)\right](t) \mathrm{dt} .
$$

To bound the first three terms of (4.19), we employ Lemma 4.4 with $\boldsymbol{y}=\boldsymbol{z} \in \mathcal{K}_{g}^{\mathrm{t}}$ and next the Young inequality $\left(A B \leq \frac{1}{4} A^{2}+B^{2}\right)$ to see

$$
\begin{aligned}
\left\|\boldsymbol{w}^{*}\right\|_{\Omega, T}^{2} \leq & \left(\left\{\sum_{n=1}^{N_{\mathrm{t}}} \int_{I_{n}} \sum_{K \in \mathcal{T}_{h}} \sum_{\alpha=1}^{2}\left(\eta_{\mathrm{R}, K, \alpha}^{n}+\eta_{\mathrm{F}, K, \alpha}^{n}\right)^{2}(t) \mathrm{dt}\right\}^{\frac{1}{2}}+\left\{\int_{0}^{T} \sum_{\alpha=1}^{2} \sum_{K \in \mathcal{T}_{h}}\left(\eta_{\mathrm{osc}, K, \alpha}^{n}\right)^{2}(t) \mathrm{dt}\right\}^{\frac{1}{2}}\right)^{2} \\
& +\frac{1}{4}\left\|\boldsymbol{w}^{*}\right\|_{\Omega, T}^{2}-\int_{0}^{T} a\left(\boldsymbol{u}-\boldsymbol{u}_{h \tau}, \boldsymbol{w}^{*}\right)(t) \mathrm{dt} .
\end{aligned}
$$

The Cauchy-Schwarz inequality and the Young inequality give

$$
-\int_{0}^{T} a\left(\boldsymbol{u}-\boldsymbol{u}_{h \tau}, \boldsymbol{w}^{*}\right)(t) \mathrm{dt} \leq\left\|\boldsymbol{u}-\boldsymbol{u}_{h \tau}\left|\left\|_{\Omega, T}\right\| \boldsymbol{w}^{*}\right|\right\|_{\Omega, T} \leq\left\|\boldsymbol{u}-\boldsymbol{u}_{h \tau} \mid\right\|_{\Omega, T}^{2}+\frac{1}{4}\left\|\boldsymbol{w}^{*}\right\|_{\Omega, T}^{2} .
$$

Finally, combining (4.20) and (4.21) with (4.12), we get $\left\|\boldsymbol{w}^{*}\right\|_{\Omega, T}^{2} \leq 4 \eta^{2}$ which is the desired result.

Combining Theorems 4.3 and 4.5, we infer

Corollary 4.6 (case $p=1$ and exact solvers). Assume the hypotheses of Theorem 4.5. Then

$$
\left\|\boldsymbol{u}-\boldsymbol{u}_{h \tau}\right\|_{\Omega, T}^{2}+\|\boldsymbol{u}-\boldsymbol{z}\|_{\Omega, T}^{2}+\left\|\left(\boldsymbol{u}-\boldsymbol{u}_{h \tau}\right)(\cdot, T)\right\|_{\Omega}^{2} \leq 5 \eta^{2} .
$$

We show now that the error measure $\|\boldsymbol{u}-\boldsymbol{z}\|_{\Omega, T}$ is linked to the temporal derivative error; unfortunately we could not obtain the (more interesting) converse estimate that would allow to control the temporal derivative error by the estimators.

Lemma 4.7. Assuming the hypotheses of Theorem 4.5 and denoting by $C_{\mu}:=2 / \min \left(\mu_{1}^{\frac{1}{2}}, \mu_{2}^{\frac{1}{2}}\right)$ we have

$$
\|\boldsymbol{u}-\boldsymbol{z}\|_{\Omega, T} \leq C_{\mu}\left(\left(\int_{0}^{T} \sum_{\alpha=1}^{2}\left\|\partial_{t}\left(u_{\alpha}-u_{\alpha h \tau}\right)\right\|_{H^{-1}(\Omega)}^{2}(t) \mathrm{dt}\right)^{\frac{1}{2}}+\left(\int_{0}^{T}\left\|\lambda_{h \tau}-\lambda\right\|_{H^{-1}(\Omega)}^{2}(t) \mathrm{dt}\right)^{\frac{1}{2}}\right)
$$

Proof. Denoting by $\boldsymbol{w}^{*}:=\boldsymbol{u}-\boldsymbol{z}$, we have

$$
\left\|\boldsymbol{w}^{*}\right\|_{\Omega, T}^{2} \leq \int_{0}^{T} \sum_{\alpha=1}^{2}\left\langle\partial_{t}\left(u_{\alpha}-u_{\alpha h \tau}\right), w_{\alpha}^{*}\right\rangle(t) \mathrm{dt}+\int_{0}^{T}\left(\lambda_{h \tau}, w_{1}^{*}-w_{2}^{*}\right)_{\Omega}(t) \mathrm{dt} .
$$


Next, we have $\left(\lambda_{h \tau}, w_{1}^{*}-w_{2}^{*}\right)_{\Omega}=\left(\lambda_{h \tau}-\lambda, w_{1}^{*}-w_{2}^{*}\right)_{\Omega}+\left(\lambda, w_{1}^{*}-w_{2}^{*}\right)_{\Omega}$, and from $(2.1) \lambda\left(u_{1}-u_{2}\right)=0$. As $\lambda \in \Psi$ and $\boldsymbol{z} \in \mathcal{K}_{g}^{\mathrm{t}}$, we have

$$
\int_{0}^{T}\left(\lambda, w_{1}^{*}-w_{2}^{*}\right)_{\Omega}(t) \mathrm{dt} \leq 0
$$

and thus

$$
\int_{0}^{T}\left(\lambda_{h \tau}, w_{1}^{*}-w_{2}^{*}\right)_{\Omega}(t) \mathrm{dt} \leq \int_{0}^{T}\left(\lambda_{h \tau}-\lambda, w_{1}^{*}-w_{2}^{*}\right)_{\Omega}(t) \mathrm{dt} .
$$

Finally,

$$
\left\|\boldsymbol{w}^{*}\right\|_{\Omega, T}^{2} \leq \int_{0}^{T} \sum_{\alpha=1}^{2}\left\langle\partial_{t}\left(u_{\alpha}-u_{\alpha h \tau}\right), w_{\alpha}^{*}\right\rangle(t) \mathrm{dt}+\int_{0}^{T}\left(\lambda_{h \tau}-\lambda, w_{1}^{*}-w_{2}^{*}\right)_{\Omega}(t) \mathrm{dt} .
$$

We denote by $A_{1}$ the first term in the right-hand side of (4.23). For $A_{1}$, we have as a result of the $H^{-1}$ norm definition and using the Cauchy-Schwarz inequality

$$
\begin{aligned}
A_{1} & \leq \int_{0}^{T} \sum_{\alpha=1}^{2} \sup _{\Phi_{\alpha} \in H_{0}^{1}(\Omega)} \frac{\left\langle\mu_{\alpha}^{-\frac{1}{2}} \partial_{t}\left(u_{\alpha}-u_{\alpha h \tau}\right), \mu_{\alpha}^{\frac{1}{2}} \Phi_{\alpha}\right\rangle}{\left\|\mu_{\alpha}^{\frac{1}{2}} \nabla \Phi_{\alpha}\right\|_{\Omega}}\left\|\mu_{\alpha}^{\frac{1}{2}} \nabla w_{\alpha}^{*}\right\|_{\Omega}(t) \mathrm{dt} \\
& =\int_{0}^{T} \sum_{\alpha=1}^{2}\left\|\mu_{\alpha}^{-\frac{1}{2}} \partial_{t}\left(u_{\alpha}-u_{\alpha h \tau}\right)\right\|_{H^{-1}(\Omega)}\left\|\mu_{\alpha}^{\frac{1}{2}} \nabla w_{\alpha}^{*}\right\|_{\Omega}(t) \mathrm{dt} \\
& \leq\left(\int_{0}^{T} \sum_{\alpha=1}^{2}\left\|\mu_{\alpha}^{-\frac{1}{2}} \partial_{t}\left(u_{\alpha}-u_{\alpha h \tau}\right)\right\|_{H^{-1}(\Omega)}^{2}(t) \mathrm{dt}\right)^{\frac{1}{2}}\left\|\boldsymbol{w}^{*}\right\|_{\Omega, T}
\end{aligned}
$$

For $A_{2}$, we employ the Cauchy-Schwarz inequality

$$
\begin{aligned}
A_{2} & =\int_{0}^{T}\left(\mu_{1}^{-\frac{1}{2}}\left(\lambda_{h \tau}-\lambda\right), \mu_{1}^{\frac{1}{2}} w_{1}^{*}\right)_{\Omega}(t) \mathrm{dt}-\int_{0}^{T}\left(\mu_{2}^{-\frac{1}{2}}\left(\lambda_{h \tau}-\lambda\right), \mu_{2}^{\frac{1}{2}} w_{2}^{*}\right)_{\Omega}(t) \mathrm{dt}, \\
& \leq C_{\mu}\left(\int_{0}^{T}\left\|\lambda_{h \tau}-\lambda\right\|_{H^{-1}(\Omega)}^{2}(t) \mathrm{dt}\right)^{\frac{1}{2}}\left\|\boldsymbol{w}^{*}\right\|_{\Omega, T} .
\end{aligned}
$$

Combining (4.23), (4.24), and (4.25), we obtain the desired result.

\subsection{An a posteriori error estimate for $p \geq 1$ and each step $k \geq 1, i \geq 0$}

In this section, we devise an a posteriori error estimate which is valid for any polynomial degree $p \geq 1$, at any time step $1 \leq n \leq N_{\mathrm{t}}$, at any semismooth Newton step $k \geq 1$, and at any algebraic step $i \geq 0$. Several difficulties arise. Contrary to the previous case of Section 4.4, the constraints (3.9) are not satisfied because the convergence is not reached. Moreover, even if they were satisfied, the solution remains nonconforming for $p \geq 2$ because $\mathcal{K}_{g h}^{p} \not \subset \mathcal{K}_{g}$ and $\Lambda_{h}^{p} \not \subset \Lambda$. Consequently, we have to work with a nonconforming space-time solution $\boldsymbol{u}_{h \tau}^{k, i} \notin \mathcal{K}_{g}^{\mathrm{t}}$ and $\lambda_{h \tau} \notin \Psi$. To cope with these difficulties, we employ the decomposition

$$
\lambda_{h}^{n, k, i}=\lambda_{h}^{n, k, i, \text { pos }}+\lambda_{h}^{n, k, i, \text { neg }} \quad \text { where } \quad \lambda_{h}^{n, k, i, \text { pos }}=\max \left\{\lambda_{h}^{n, k, i}, 0\right\} \quad \text { and } \quad \lambda_{h}^{n, k, i, \text { neg }}=\min \left\{\lambda_{h}^{n, k, i}, 0\right\} .
$$

We also introduce a potential $\tilde{s}_{h \tau}^{k, i}:=\left(\tilde{s}_{1, h \tau}^{k, i}, \tilde{s}_{2, h \tau}^{k, i}\right) \in \mathcal{K}_{g}^{\mathrm{t}}$ as a piecewise affine and continuous function in time over the whole time interval $] 0, T\left[\right.$, verifying $\tilde{s}_{1, h \tau}^{k, i}(t)-\tilde{s}_{2, h \tau}^{k, i}(t) \geq 0$ for all $\left.t \in\right] 0, T[$.

Remark 4.8. A possible construction for $\tilde{\boldsymbol{s}}_{h \tau}^{k, i} \in \mathcal{K}_{g}^{\mathrm{t}}$ can be derived from the stationary version presented in [28, Sec. 5.2]. We recall the main ingredients here. First, construct $\boldsymbol{s}_{h}^{n, k, i} \in \mathcal{K}_{g h}^{p}$ at each node $\boldsymbol{x}_{l} \in \mathcal{V}^{p, \mathrm{i}}$ 
by

$$
\boldsymbol{s}_{h}^{n, k, i}\left(\boldsymbol{x}_{l}\right):= \begin{cases}\boldsymbol{u}_{h}^{n, k, i}\left(\boldsymbol{x}_{l}\right)=\left(u_{1 h}^{n, k, i}\left(\boldsymbol{x}_{l}\right), u_{2 h}^{n, k, i}\left(\boldsymbol{x}_{l}\right)\right) & \text { if }\left(u_{1 h}^{n, k, i}-u_{2 h}^{n, k, i}\right)\left(\boldsymbol{x}_{l}\right) \geq 0, \\ \left(\frac{u_{1 h}^{n, k, i}\left(\boldsymbol{x}_{l}\right)+u_{2 h}^{n, k, i}\left(\boldsymbol{x}_{l}\right)}{2}, \frac{u_{1 h}^{n, k, i}\left(\boldsymbol{x}_{l}\right)+u_{2 h}^{n, k, i}\left(\boldsymbol{x}_{l}\right)}{2}\right) & \text { if }\left(u_{1 h}^{n, k, i}-u_{2 h}^{n, k, i}\right)\left(\boldsymbol{x}_{l}\right)<0 .\end{cases}
$$

For $p=1$, this gives $s_{1 h}^{n, k, i} \geq s_{2 h}^{n, k, i}$ everywhere, so we can set $\tilde{\boldsymbol{s}}_{h}^{n, k, i}:=\boldsymbol{s}_{h}^{n, k, i}$.

For $p \geq 2$, imposing $s_{1 h}^{n, k, i}\left(\boldsymbol{x}_{l}\right) \geq s_{2 h}^{n, k, i}\left(\boldsymbol{x}_{l}\right)$ in the Lagrange nodes is not sufficient to have $s_{1 h}^{n, k, i} \geq s_{2 h}^{n, k, i}$. So, we add to $s_{1 h}^{n, k, i}-s_{2 h}^{n, k, i}$ bubble functions, first on all edges, then on all triangles. This builds $\tilde{\boldsymbol{s}}_{h}^{n, k, i}$ such that $\tilde{s}_{1 h}^{n, k, i} \geq \tilde{s}_{2 h}^{n, k, i}$, and we define $\tilde{s}_{h \tau}^{n, k, i}$ from $\tilde{\boldsymbol{s}}_{h}^{n, k, i}$ as its corresponding space-time function.

Definition 4.9. Let $C_{\Omega, \mu}:=h_{\Omega} C_{\mathrm{PF}}\left(\frac{1}{\mu_{1}}+\frac{1}{\mu_{2}}\right)^{\frac{1}{2}}$. For all $1 \leq n \leq N_{\mathrm{t}}$, we define the error estimators

$$
\begin{aligned}
\eta_{\mathrm{R}, K, \alpha}^{n, k, i}(t) & :=h_{\Omega} C_{\mathrm{PF}} \mu_{\alpha}^{-\frac{1}{2}}\left\|\tilde{f}_{\alpha}^{n}-\partial_{t} \tilde{s}_{\alpha h \tau}^{n, k, i}-\nabla \cdot \boldsymbol{\sigma}_{\alpha h}^{n, k, i}-(-1)^{\alpha} \lambda_{h}^{n, k, i}\right\|_{K}(t), \\
\eta_{\mathrm{F}, K, \alpha}^{n, k, i}(t) & :=\left\|\mu_{\alpha}^{\frac{1}{2}} \nabla \tilde{s}_{\alpha h \tau}^{n, k, i}+\mu_{\alpha}^{-\frac{1}{2}} \boldsymbol{\sigma}_{\alpha h}^{n, k, i}\right\|_{K}(t), \\
\eta_{\mathrm{C}, K}^{n, k, i, \operatorname{pos}}(t) & :=2\left(\lambda_{h}^{n, k, i, \operatorname{pos}}, u_{1 h \tau}^{n, k, i}-u_{2 h \tau}^{n, k, i}\right)_{K}(t), \\
\eta_{\text {nonc }, 1, K}^{n, k, i}(t) & :=C_{\Omega, \mu}\left\|\lambda_{h}^{n, k, i, \operatorname{neg}}\right\|_{K}(t), \\
\eta_{\text {nonc }, 2, K}^{n, k, i}(t) & :=\left\|\tilde{s}_{h \tau}^{n, k, i}-\boldsymbol{u}_{h \tau}^{n, k, i}\right\|_{K}(t), \\
\eta_{\text {nonc }, 3, K}^{n, k, i}(t) & :=2\left(\lambda_{h}^{n, k, i, \operatorname{pos}},\left(\tilde{s}_{1 h \tau}^{n, k, i}-u_{1 h \tau}^{n, k, i}\right)-\left(\tilde{s}_{2 h \tau}^{n, k, i}-u_{2 h \tau}^{n, k, i}\right)\right)_{K}(t), \\
\eta_{\mathrm{osc}, K, \alpha}^{n}(t) & :=C_{\mathrm{PF}} h_{\Omega} \mu_{\alpha}^{-\frac{1}{2}}\left\|f_{\alpha}-\tilde{f}_{\alpha}^{n}\right\|_{K} .
\end{aligned}
$$

We observe that the estimators given by Definition 4.9 are slightly different from the ones provided in Definition 4.1. Indeed, in the estimators $\eta_{\mathrm{R}, \mathrm{K}, \alpha}^{n, k, i}$ and $\eta_{\mathrm{F}, \mathrm{K}, \alpha}^{n, k, i}$, there appears $\tilde{s}_{\alpha h \tau}^{n, k, i}$ in place of $u_{\alpha h \tau}^{n, k, i}$, and $h_{\Omega}$ instead of $h_{K}$. The constraint estimator $\eta_{\mathrm{C}, K}^{n, k, i, \text { pos }}$ is as in Definition 4.1 (remember that $\lambda_{h}^{n} \geq 0$ at convergence for $p=1)$ and expresses that $\lambda_{h}^{n, k, i}\left(u_{1 h \tau}^{n, k, i}-u_{2 h \tau}^{n, k, i}\right)=0$ is not valid everywhere. Next, $\eta_{\text {nonc }, 1, K}^{n, k,}$, $\eta_{\text {nonc }, 2, K}^{n, k, i}$, and $\eta_{\text {nonc,3,K }}^{n, k, i}$ are nonconformity estimators expressing the possible negativity of the discrete Lagrange multiplier and measuring how far the potential reconstruction $\tilde{s}_{h \tau}^{n, k, i}$ is from the displacements $\boldsymbol{u}_{h \tau}^{n, k, i}$.

Theorem 4.10 (case $p \geq 1$ and inexact solvers). Let $\boldsymbol{u} \in \mathcal{K}_{g}^{\mathrm{t}}$ be the exact solution given by (1.2) and let $\boldsymbol{u}_{h \tau}^{k, i} \notin \mathcal{K}_{g}^{\mathrm{t}}$ be the approximate solution issued from inexact linearization step $k \geq 1$ and algebraic solvers step $i \geq 0$ at each time step $1 \leq n \leq N_{\mathrm{t}}$. Consider the total equilibrated flux reconstruction $\sigma_{\alpha h \tau}^{k, i} \in$ $L^{2}(0, T, \mathbf{H}(\operatorname{div}, \Omega))$ given by (4.4) and (4.6). Let $\tilde{\boldsymbol{s}}_{h \tau}^{k, i} \in \mathcal{K}_{g}^{\mathrm{t}}$ and consider the estimators of Definition 4.9. Then, for

$$
\begin{aligned}
\left(\bar{\eta}^{k, i}\right)^{2}:= & \left(\left\{\sum_{n=1}^{N_{\mathrm{t}}} \int_{I_{n}} \sum_{K \in \mathcal{T}_{h}} \sum_{\alpha=1}^{2}\left(\eta_{\mathrm{R}, \mathrm{K}, \alpha}^{n, k, i}\right)^{2}(t) \mathrm{dt}\right\}^{\frac{1}{2}}+\left\{\sum_{n=1}^{N_{\mathrm{t}}} \int_{I_{n}} \sum_{K \in \mathcal{T}_{h}} \sum_{\alpha=1}^{2}\left(\eta_{\mathrm{F}, \mathrm{K}, \alpha}^{n, k, i}\right)^{2}(t) \mathrm{dt}\right\}^{\frac{1}{2}}\right. \\
& \left.+\left\{\sum_{n=1}^{N_{\mathrm{t}}} \int_{I_{n}} \sum_{K \in \mathcal{T}_{h}}\left(\eta_{\text {nonc }, 1, K}^{n, k, i}\right)^{2}(t) \mathrm{dt}\right\}^{\frac{1}{2}}+\left\{\sum_{n=1}^{N_{\mathrm{t}}} \int_{I_{n}} \sum_{K \in \mathcal{T}_{h}} \sum_{\alpha=1}^{2}\left(\eta_{\mathrm{osc}, K, \alpha}^{n}\right)^{2}(t) \mathrm{dt}\right\}^{\frac{1}{2}}\right)^{2} \\
& +\sum_{n=1}^{N_{\mathrm{t}}} \int_{I_{n}} \sum_{K \in \mathcal{T}_{h}} \eta_{\mathrm{C}, K}^{n, k, i, \operatorname{pos}}(t) \mathrm{dt}+\sum_{n=1}^{N_{\mathrm{t}}} \int_{I_{n}} \sum_{K \in \mathcal{T}_{h}} \eta_{\text {nonc }, 3, K}^{n, k, i}(t) \mathrm{dt}+\left\|\left(\boldsymbol{u}-\tilde{\boldsymbol{s}}_{h \tau}^{n, k, i}\right)(\cdot, 0)\right\|_{\Omega}^{2},
\end{aligned}
$$


we have the a posteriori error estimate

$$
\left\|\boldsymbol{u}-\boldsymbol{u}_{h \tau}^{k, i}\right\|_{\Omega, T} \leq \tilde{\eta}^{k, i}:=\bar{\eta}^{k, i}+\left\{\sum_{n=1}^{N_{\mathrm{t}}} \int_{I_{n}} \sum_{K \in \mathcal{T}_{h}}\left(\eta_{\text {nonc }, 2, K}^{n, k, i}\right)^{2}(t) \mathrm{dt}\right\}^{\frac{1}{2}} .
$$

Proof. We start by the triangle inequality, leading to

$$
\left\|\boldsymbol{u}-\boldsymbol{u}_{h \tau}^{k, i}\right\|\left\|_{\Omega, T} \leq\right\| \boldsymbol{u}-\tilde{s}_{h \tau}^{k, i}\|\|_{\Omega, T}+\left\|\tilde{s}_{h \tau}^{k, i}-\boldsymbol{u}_{h \tau}^{k, i}\right\| \|_{\Omega, T} .
$$

The second term of (4.29) immediately equals to

$$
\left\|\tilde{s}_{h \tau}^{k, i}-\boldsymbol{u}_{h \tau}^{k, i}\right\|_{\Omega, T}^{2}=\sum_{n=1}^{N_{\mathrm{t}}} \int_{I_{n}} \sum_{K \in \mathcal{T}_{h}}\left(\eta_{\text {nonc }, 2, K}^{n, k, i}\right)^{2}(t) \mathrm{dt}
$$

Next, observe that

$$
\left\|\boldsymbol{u}-\tilde{\boldsymbol{s}}_{h \tau}^{k, i}\right\|_{\Omega, T}^{2} \leq\|\| \boldsymbol{u}-\tilde{\boldsymbol{s}}_{h \tau}^{k, i}\|\|_{\Omega, T}^{2}+\frac{1}{2}\left\|\left(\boldsymbol{u}-\tilde{\boldsymbol{s}}_{h \tau}^{k, i}\right)(\cdot, T)\right\|_{\Omega}^{2} .
$$

Employing the fact that

$$
\frac{1}{2}\left\|\left(\boldsymbol{u}-\tilde{\boldsymbol{s}}_{h \tau}^{k, i}\right)(\cdot, T)\right\|_{\Omega}^{2}=\frac{1}{2}\left\|\left(\boldsymbol{u}-\tilde{\boldsymbol{s}}_{h \tau}^{k, i}\right)(\cdot, 0)\right\|_{\Omega}^{2}+\int_{0}^{T}\left\langle\partial_{t}\left(\boldsymbol{u}-\tilde{\boldsymbol{s}}_{h \tau}^{k, i}\right), \boldsymbol{u}-\tilde{\boldsymbol{s}}_{h \tau}^{k, i}\right\rangle(t) \mathrm{dt},
$$

we have

$$
\begin{aligned}
\left\|\boldsymbol{u}-\tilde{\boldsymbol{s}}_{h \tau}^{k, i}\right\|_{\Omega, T}^{2} \leq & \sum_{\alpha=1}^{2} \int_{0}^{T} \mu_{\alpha}\left(\nabla\left(u_{\alpha}-\tilde{s}_{\alpha h \tau}^{k, i}\right), \boldsymbol{\nabla}\left(u_{\alpha}-\tilde{s}_{\alpha h \tau}^{k, i}\right)\right)_{\Omega}(t) \mathrm{dt} \\
& +\sum_{\alpha=1}^{2} \int_{0}^{T}\left\langle\partial_{t}\left(u_{\alpha}-\tilde{s}_{\alpha h \tau}^{k, i}\right), u_{\alpha}-\tilde{s}_{\alpha h \tau}^{k, i}\right\rangle(t) \mathrm{dt}+\frac{1}{2}\left\|\left(\boldsymbol{u}-\tilde{\boldsymbol{s}}_{h \tau}^{k, i}\right)(\cdot, 0)\right\|_{\Omega}^{2} .
\end{aligned}
$$

We now use the weak formulation (1.2) with $\boldsymbol{v}=\tilde{\boldsymbol{s}}_{h \tau}^{k, i} \in \mathcal{K}_{g}^{\mathrm{t}}$ and we add and subtract $\sum_{\alpha=1}^{2} \int_{0}^{T}\left(\tilde{f}_{\alpha}^{n}, u_{\alpha}-\right.$ $\left.\tilde{s}_{\alpha h \tau}^{k, i}\right)_{\Omega}(t) \mathrm{dt}$ to get

$$
\begin{aligned}
\left\|\boldsymbol{u}-\tilde{\boldsymbol{s}}_{h \tau}^{k, i}\right\| \|_{\Omega, T}^{2} \leq & \sum_{\alpha=1}^{2} \int_{0}^{T}\left(\tilde{f}_{\alpha}^{n}-\partial_{t} \tilde{s}_{\alpha h \tau}^{k, i}, u_{\alpha}-\tilde{s}_{\alpha h \tau}^{k, i}\right)_{\Omega}(t)-\sum_{\alpha=1}^{2} \int_{0}^{T} \mu_{\alpha}\left(\boldsymbol{\nabla} \tilde{s}_{\alpha h \tau}^{k, i}, \boldsymbol{\nabla}\left(u_{\alpha}-\tilde{s}_{\alpha h \tau}^{k, i}\right)\right)_{\Omega}(t) \mathrm{dt} \\
& +\sum_{\alpha=1}^{2} \int_{0}^{T}\left(f_{\alpha}-\tilde{f}_{\alpha}^{n}, u_{\alpha}-\tilde{s}_{\alpha h \tau}^{k, i}\right)_{\Omega}(t) \mathrm{dt}+\frac{1}{2}\left\|\left(\boldsymbol{u}-\tilde{\boldsymbol{s}}_{h \tau}^{k, i}\right)(\cdot, 0)\right\|_{\Omega}^{2} .
\end{aligned}
$$

Adding and subtracting $\sum_{\alpha=1}^{2} \int_{0}^{T}\left((-1)^{\alpha} \lambda_{h \tau}^{k, i}, u_{\alpha}-\tilde{s}_{\alpha h \tau}^{k, i}\right)_{\Omega}(t) \mathrm{dt}$ and $\sum_{\alpha=1}^{2} \int_{0}^{T}\left(\sigma_{\alpha h \tau}^{k, i}, \nabla\left(u_{\alpha}-\tilde{s}_{\alpha h \tau}^{k, i}\right)\right)_{\Omega}(t) \mathrm{dt}$ with $\boldsymbol{\sigma}_{\alpha h \tau}^{k, i} \in L^{2}(0, T ; \mathbf{H}(\operatorname{div}, \Omega))$ and using the Green formula with $\left(u_{\alpha}-\tilde{s}_{\alpha h \tau}^{k, i}\right)(t) \in H_{0}^{1}(\Omega)$ a.e. $\left.t \in\right] 0, T[$, we obtain

$$
\left\|\boldsymbol{u}-\tilde{\boldsymbol{s}}_{h \tau}^{k, i}\right\|_{\Omega, T}^{2} \leq A_{1}+A_{2}+A_{3}+A_{4}+\frac{1}{2}\left\|\left(\boldsymbol{u}-\tilde{\boldsymbol{s}}_{h \tau}^{k, i}\right)(\cdot, 0)\right\|_{\Omega}^{2}
$$


with

$$
\begin{aligned}
& A_{1}:=\sum_{\alpha=1}^{2} \int_{0}^{T}\left(\tilde{f}_{\alpha}^{n}-\partial_{t} \tilde{s}_{\alpha h \tau}^{k, i}-\nabla \cdot \boldsymbol{\sigma}_{\alpha h \tau}^{k, i}-(-1)^{\alpha} \lambda_{h \tau}^{k, i}, u_{\alpha}-\tilde{s}_{\alpha h \tau}^{k, i}\right)_{\Omega}(t) \mathrm{dt}, \\
& A_{2}:=-\sum_{\alpha=1}^{2} \int_{0}^{T}\left(\mu_{\alpha}^{\frac{1}{2}} \nabla \tilde{s}_{\alpha h \tau}^{k, i}+\mu_{\alpha}^{-\frac{1}{2}} \boldsymbol{\sigma}_{\alpha h \tau}^{k, i}, \mu_{\alpha}^{\frac{1}{2}} \nabla\left(u_{\alpha}-\tilde{s}_{\alpha h \tau}^{k, i}\right)\right)_{\Omega}(t) \mathrm{dt}, \\
& A_{3}:=\sum_{\alpha=1}^{2} \int_{0}^{T}\left((-1)^{\alpha} \lambda_{h \tau}^{k, i}, u_{\alpha}-\tilde{s}_{\alpha h \tau}^{k, i}\right)_{\Omega}(t) \mathrm{dt}, \\
& A_{4}:=\sum_{\alpha=1}^{2} \int_{0}^{T}\left(f_{\alpha}-\tilde{f}_{\alpha}^{n}, u_{\alpha}-\tilde{s}_{\alpha h \tau}^{k, i}\right)_{\Omega}(t) \mathrm{dt} .
\end{aligned}
$$

To bound $A_{1}, A_{2}$, and $A_{4}$ we proceed as follows. We apply the Cauchy-Schwarz inequality and next the Poincaré-Friedrichs inequality (4.11a) to get

$$
\begin{aligned}
& A_{1} \leq\left(\sum_{n=1}^{N_{\mathrm{t}}} \int_{I_{n}} \sum_{\alpha=1}^{2} \sum_{K \in \mathcal{T}_{h}}\left(\eta_{\mathrm{R}, \mathrm{K}, \alpha}^{n, k, i}\right)^{2}(t) \mathrm{dt}\right)^{\frac{1}{2}}\left\|\boldsymbol{u}-\tilde{\boldsymbol{s}}_{h \tau}^{n, k, i}\right\| \|_{\Omega, T}, \\
& A_{2} \leq\left(\sum_{n=1}^{N_{\mathrm{t}}} \int_{I_{n}} \sum_{\alpha=1}^{2} \sum_{K \in \mathcal{T}_{h}}\left(\eta_{\mathrm{F}, \mathrm{K}, \alpha}^{n, k, i}\right)^{2}(t) \mathrm{dt}\right)^{\frac{1}{2}}\left\|\boldsymbol{u}-\tilde{\boldsymbol{s}}_{h \tau}^{n, k, i}\right\| \|_{\Omega, T}, \\
& A_{4} \leq\left(\sum_{n=1}^{N_{\mathrm{t}}} \int_{I_{n}} \sum_{\alpha=1}^{2} \sum_{K \in \mathcal{T}_{h}}\left(\eta_{\mathrm{osc}, K, \alpha}^{n}\right)^{2}(t) \mathrm{dt}\right)^{\frac{1}{2}}\left\|\boldsymbol{u}-\tilde{\boldsymbol{s}}_{h \tau}^{n, k, i}\right\| \|_{\Omega, T} .
\end{aligned}
$$

It remains to bound the term $A_{3}$. Observe that

$$
A_{3}=-\int_{0}^{T} b\left(\boldsymbol{u}-\tilde{\boldsymbol{s}}_{h \tau}^{k, i}, \lambda_{h \tau}^{k, i, \mathrm{neg}}\right)(t) \mathrm{dt}-\int_{0}^{T} b\left(\boldsymbol{u}-\tilde{\boldsymbol{s}}_{h \tau}^{k, i}, \lambda_{h \tau}^{k, i, \mathrm{pos}}\right)(t) \mathrm{dt}
$$

Next, adding and subtracting $b\left(\boldsymbol{u}_{h \tau}^{k, i}, \lambda_{h \tau}^{k, i, \text { pos }}\right)$ and noting that $-b\left(\boldsymbol{u}, \lambda_{h \tau}^{k, i, \text { pos }}\right) \leq 0$ since $\boldsymbol{u} \in \mathcal{K}_{g}^{\mathrm{t}}$ and $\lambda_{h \tau}^{k, i, \text { pos }}(t) \geq 0$ for all $\left.t \in\right] 0, T[$, we have

$$
A_{3} \leq A_{31}+A_{32}+A_{33}
$$

with $A_{31}:=-\int_{0}^{T} b\left(\boldsymbol{u}-\tilde{\boldsymbol{s}}_{h \tau}^{k, i}, \lambda_{h \tau}^{k, i, \mathrm{neg}}\right)(t) \mathrm{dt}, A_{32}:=\int_{0}^{T} b\left(\tilde{\boldsymbol{s}}_{h \tau}^{k, i}-\boldsymbol{u}_{h \tau}^{k, i}, \lambda_{h \tau}^{k, i, \operatorname{pos}}\right)(t) \mathrm{dt}, A_{33}:=\int_{0}^{T} b\left(\boldsymbol{u}_{h \tau}^{k, i}, \lambda_{h \tau}^{k, i, \operatorname{pos}}\right)(t) \mathrm{dt}$.

The Cauchy-Schwarz inequality and the Poincaré-Friedrichs inequality (4.11a) yield

$$
A_{31} \leq\left(\sum_{n=1}^{N_{\mathrm{t}}} \int_{I_{n}} \sum_{K \in \mathcal{T}_{h}}\left(\eta_{\text {nonc, }, 1, K}^{n, k, i}\right)^{2}(t) \mathrm{dt}\right)^{\frac{1}{2}}\left\|\boldsymbol{u}-\tilde{\boldsymbol{s}}_{h \tau}^{n, k, i}\right\| \|_{\Omega, T} .
$$

Next, we have

$$
A_{32}=\frac{1}{2} \sum_{n=1}^{N_{\mathrm{t}}} \int_{I_{n}} \sum_{K \in \mathcal{T}_{h}} \eta_{\text {nonc }, 3, K}^{n, k, i}(t) \mathrm{dt}, \quad A_{33}=\frac{1}{2} \sum_{n=1}^{N_{\mathrm{t}}} \int_{I_{n}} \sum_{K \in \mathcal{T}_{h}} \eta_{\mathrm{C}, K}^{n, k, i, \operatorname{pos}}(t) \mathrm{dt} .
$$

Finally, combining (4.31)-(4.37), employing the Young inequality $a b \leq \frac{1}{2}\left(a^{2}+b^{2}\right),(a, b) \geq 0$, and using (4.30) provides the desired result. 


\section{$5 \quad$ Error components and adaptive inexact algorithm}

In Section 4.5, we have derived an a posteriori error estimate between the exact solution and approximate solution at each semismooth Newton step $k \geq 1$ and each algebraic iterative solver step $i \geq 0$. We now provide an a posteriori error estimate distinguishing the different error components (discretization $\eta_{\text {disc }}^{n, k, i}$, semismooth linearization $\eta_{\mathrm{lin}}^{n, k, i}$, and linear algebra $\eta_{\mathrm{alg}}^{n, k, i}$ ) for any $p \geq 1$. To identify these components, we note that $\eta_{\text {alg }}^{n, k, i}$ must vanish when $i \rightarrow \infty, \eta_{\text {lin }}^{n, k, i}$ must vanish when $i$ and $k \rightarrow \infty$, and $\eta_{\text {disc }}^{n, k, i}$ must vanish when $i, k \rightarrow \infty$ and $h$ and $\Delta t \rightarrow 0$. The components are adapted from the ideas of [28, Sec. 5.4]. This will allow us to define the adaptive inexact semismooth Newton algorithm for instationary variational inequalities.

\subsection{Distinguishing the error components for $p \geq 1$}

Let $n \in\left\{1, \ldots, N_{\mathrm{t}}\right\}$. In this section, the dependency on the time variable is not shown explicitely. First, using (4.4), the triangle inequality reads

$$
\eta_{\mathrm{F}, \mathrm{K}, \alpha}^{n, k, i} \leq \underbrace{\left\|\mu_{\alpha}^{\frac{1}{2}} \nabla \tilde{s}_{\alpha h \tau}^{n, k, i}+\mu_{\alpha}^{-\frac{1}{2}} \boldsymbol{\sigma}_{\alpha h, \mathrm{disc}}^{n, k, i}\right\|_{K}}_{\text {discretization }}+\underbrace{\left\|\mu_{\alpha}^{-\frac{1}{2}} \boldsymbol{\sigma}_{\alpha h, \mathrm{alg}}^{n, k, i}\right\|_{K}}_{\text {algebra }},
$$

which separates algebraic and discretization contributions of the error. Define

$$
\eta_{\mathrm{disc}, \mathrm{F}, K, \alpha}^{n, k, i}:=\left\|\mu_{\alpha}^{\frac{1}{2}} \nabla \tilde{s}_{\alpha h \tau}^{n, k, i}+\mu_{\alpha}^{-\frac{1}{2}} \boldsymbol{\sigma}_{\alpha h, \mathrm{disc}}^{n, k, i}\right\|_{K}, \eta_{\mathrm{alg}, K, \alpha}^{n, k, i}:=\left\|\mu_{\alpha}^{-\frac{1}{2}} \boldsymbol{\sigma}_{\alpha h, \mathrm{alg}}^{n, k, i}\right\|_{K} .
$$

Case $p=1$ : let $\beta \in\{1,2,3\}$. The estimators $\eta_{\text {nonc }, \beta, K}^{n, k, i}$ can be interpreted as semismooth linearization estimators. Indeed, at convergence for $k$ and $i \rightarrow \infty, \lambda_{h}^{n, k, i, \operatorname{pos}}=\lambda_{h}^{n} \in \Lambda, \lambda_{h}^{n, k, i, \text { neg }}=0$, and $\tilde{\boldsymbol{s}}_{h}^{n, k, i}=\boldsymbol{u}_{h}^{n} \in$ $\mathcal{K}_{g}$. Thus $\eta_{\text {nonc, }, \beta, K}^{n, k, i}$ vanish at convergence, and we can set:

$$
\eta_{\operatorname{lin}, \beta, K}^{n, k, i}:=\eta_{\text {nonc }, \beta, K}^{n, k, i} \quad \text { for } \beta=1,2,3 \quad \text { when } p=1 .
$$

The estimators $\eta_{\mathrm{C}, K}^{n, k, i, \operatorname{pos}}$ are attributed to the discretization component, as they vanish only when $h, \Delta t \rightarrow 0$.

Case $p \geq 2$ : the procedure is more intricate. We employ the triangle inequality and the construction of Remark 4.8 to get

$$
\eta_{\text {nonc }, 2, K}^{n, k, i} \leq \underbrace{\left\|\tilde{\boldsymbol{s}}_{h \tau}^{n, k, i}-\boldsymbol{s}_{h \tau}^{n, k, i}\right\| \|_{K}}_{\text {discretization }}+\underbrace{\left\|\boldsymbol{s}_{h \tau}^{n, k, i}-\boldsymbol{u}_{h \tau}^{n, k, i}\right\|_{K}}_{\text {linearization }} .
$$

The first term in (5.4) vanishes assuming $\boldsymbol{u}_{h \tau}^{k, i} \rightarrow \boldsymbol{u} \in \mathcal{K}_{g}^{\mathrm{t}}$, when $h, \Delta t \rightarrow 0$ and $k, i \rightarrow \infty$. Using (4.27), the second term vanishes when $k$ and $i \rightarrow \infty$. Next, using (4.26), we treat the estimators $\eta_{\mathrm{C}, K}^{n, k, i, \operatorname{pos}}$,

$$
\sum_{K \in \mathcal{T}_{h}} \eta_{\mathrm{C}, K}^{n, k, i, \operatorname{pos}}=2 \underbrace{\left(-\lambda_{h}^{n, k, i, \mathrm{neg}}, u_{1 h \tau}^{n, k, i}-u_{2 h \tau}^{n, k, i}\right)_{\Omega}}_{\text {discretization }}+2 \underbrace{\left(\lambda_{h}^{n, k, i}, u_{1 h \tau}^{n, k, i}-u_{2 h \tau}^{n, k, i}\right)_{\Omega}}_{\text {linearization }} .
$$

The first term of (5.5) is a discretization estimator, as it vanishes assuming that $\lambda_{h \tau}^{k, i} \rightarrow \lambda \in \Psi$, when $h, \Delta t \rightarrow 0$, and $k, i \rightarrow \infty$. By virtue of (3.9) and (3.4), the second term of (5.5) is a linearization estimator, as it vanishes when $k, i \rightarrow \infty$. Next, we decompose $\lambda_{h}^{n, k, i}=\tilde{\lambda}_{h}^{n, k, i, \text { pos }}+\tilde{\lambda}_{h}^{n, k, i, \text { neg }}$, with

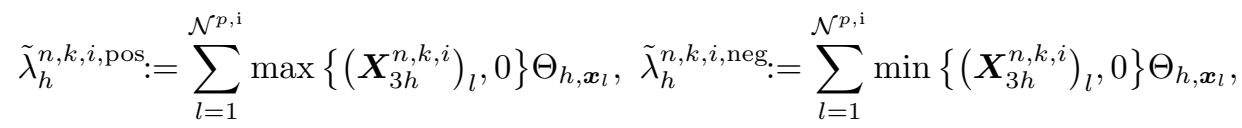

so that $\tilde{\lambda}_{h}^{n, k, i, \text { pos }}$ and $-\tilde{\lambda}_{h}^{n, k, i, \text { neg }} \in \Lambda_{h}^{p}$ (recall that $\lambda_{h}^{k, i, \text { pos }}, \lambda_{h}^{k, i, \text { neg }} \notin X_{h}^{p}$ in general), and

$$
\eta_{\text {nonc }, 1, K}^{n, k, i} \leq C_{\Omega, \mu}(\underbrace{\left\|\lambda_{h}^{n, k, i, \text { neg }}-\tilde{\lambda}_{h}^{n, k, i, n e g}\right\|_{K}}_{\text {discretization }}+\underbrace{\left\|\tilde{\lambda}_{h}^{n, k, i, \text { neg }}\right\|_{K}}_{\text {linearization }})
$$


where $\tilde{\lambda}_{h}^{n, k, i, \text { neg }} \rightarrow 0$ when $k, i \rightarrow \infty$, and $\lambda_{h}^{n, k, i, \text { neg }} \rightarrow 0$ when $h \rightarrow 0$. Finally, similarly to (5.4), the term $\eta_{\text {nonc,3,K }}^{n, k, i}$ is decomposed into two components, one with $s_{\alpha h \tau}^{n, k, i}-u_{\alpha h \tau}^{n, k, i}$, the other with $\tilde{s}_{\alpha h \tau}^{n, k, i}-s_{\alpha h \tau}^{n, k, i}$.

Regrouping this last term and (5.4)-(5.6), we define the semismooth linearization estimators for $p \geq 2$

$$
\begin{aligned}
\eta_{\operatorname{lin}, i, K}^{n, k, i} & :=C_{\Omega, \mu}\left\|\tilde{\lambda}_{h}^{n, k, i, \operatorname{neg}}\right\|_{K}, \quad \eta_{\ln , 2, K}^{n, k, i}:=\left\|s_{h \tau}^{n, k, i}-\boldsymbol{u}_{h \tau}^{n, k, i}\right\|_{K}, \\
\eta_{\operatorname{lin}, 3, K}^{n, k, i} & :=2\left(\lambda_{h}^{n, k, i, \operatorname{pos}}, s_{1 h \tau}^{n, k, i}-u_{1 h \tau}^{n, k, i}-\left(s_{2 h \tau}^{n, k, i}-u_{2 h \tau}^{n, k, i}\right)\right)_{K}, \\
\eta_{\operatorname{lin}, C, K}^{n, k, i} & :=2\left(\lambda_{h}^{n, k, i}, u_{1 h \tau}^{n, k, i}-u_{2 h \tau}^{n, k, i}\right)_{K} .
\end{aligned}
$$

We also define the discretization estimators for $p \geq 2$

$$
\begin{aligned}
\eta_{\text {disc, },, K}^{n, k, i} & :=C_{\Omega, \mu}\left\|\lambda_{h}^{n, k, i, \text { neg }}-\tilde{\lambda}_{h}^{n, k, i, \text { neg }}\right\|_{K}, \quad \eta_{\text {disc }, 2, K}^{n, k, i}:=\|\| \tilde{s}_{h \tau}^{n, k, i}-s_{h \tau}^{n, k, i}\|\|_{K}, \\
\eta_{\text {disc }, 3, K}^{n, k, i} & :=2\left(\lambda_{h}^{n, k, i, \operatorname{pos}}, \tilde{s}_{1 h \tau}^{n, k, i}-s_{1 h \tau}^{n, k, i}-\left(\tilde{s}_{2 h \tau}^{n, k, i}-s_{2 h \tau}^{n, k, i}\right)\right)_{K}, \\
\eta_{\text {disc }, C, K}^{n, k, i} & :=2\left(-\lambda_{h}^{n, k, i, \text { neg }}, u_{1 h \tau}^{n, k, i}-u_{2 h \tau}^{n, k, i}\right)_{K}
\end{aligned}
$$

Definition 5.1. Using (5.2), (5.3) for $p=1$, and (5.2), (5.7), (5.8) for $p \geq 2$, we define the initial error estimator $\eta_{\mathrm{init}}$, the local-in-time algebraic error estimator $\eta_{\mathrm{alg}}^{n, k, i}$, the local-in-time discretization error estimator $\eta_{\mathrm{disc}}^{n, k, i}$, and the local-in-time semismooth linearization error estimator $\eta_{\mathrm{lin}}^{n, k, i}$, respectively, by (5.9), (5.10) for $p=1$, and (5.9), (5.11) for $p \geq 2$.

$$
\eta_{\text {init }}:=\left\|\left(\boldsymbol{u}-\tilde{\boldsymbol{s}}_{h \tau}^{k, i}\right)(\cdot, 0)\right\|_{\Omega} .
$$

Case $p=1$ :

$$
\begin{aligned}
& \eta_{\mathrm{disc}}^{n, k, i}:=\left\{\int _ { I _ { n } } \sum _ { K \in \mathcal { T } _ { h } } \left(5\left[\sum_{\alpha=1}^{2}\left(\eta_{\mathrm{R}, \mathrm{K}, \alpha}^{n, k, i}\right)^{2}+\left(\eta_{\mathrm{disc}, \mathrm{F}, K, \alpha}^{n, k, i}\right)^{2}+\left(\eta_{\mathrm{osc}, K, \alpha}^{n}\right)^{2}\right]\right.\right. \\
&\left.\left.+\left|\eta_{\mathrm{C}, K}^{n, k, i, \mathrm{pos}}\right|\right)(t) \mathrm{dt}\right\}^{\frac{1}{2}}, \\
& \eta_{\mathrm{lin}}^{n, k, i}:=2^{\frac{1}{2}}\left\{\int_{I_{n}} \sum_{K \in \mathcal{T}_{h}}\left(5\left(\eta_{\mathrm{lin}, 1, K}^{n, k, i}\right)^{2}+\left(\eta_{\mathrm{lin}, 2, K}^{n, k, i}\right)^{2}+5\left|\eta_{\operatorname{lin}, 3, K}^{n, k, i}\right|\right)(t) \mathrm{dt}\right\}^{\frac{1}{2}}, \\
& \eta_{\mathrm{alg}}^{n, k, i}:=\left\{5 \Delta t_{n} \sum_{K \in \mathcal{T}_{h}} \sum_{\alpha=1}^{2}\left(\eta_{\mathrm{alg}, K, \alpha}^{n, k, i}\right)^{2}\right\}^{\frac{1}{2}} .
\end{aligned}
$$

Case $p \geq 2$ :

$$
\begin{aligned}
\eta_{\mathrm{disc}}^{n, k, i}:= & 2^{\frac{1}{2}}\left\{\int _ { I _ { n } } \sum _ { K \in \mathcal { T } _ { h } } \left[\sum_{\alpha=1}^{2} 6\left(\left(\eta_{\mathrm{R}, \mathrm{K}, \alpha}^{n, k, i}\right)^{2}+\left(\eta_{\mathrm{disc}, \mathrm{F}, K, \alpha}^{n, k, i}\right)^{2}+\left(\eta_{\mathrm{osc}, K, \alpha}^{n}\right)^{2}\right)\right.\right. \\
& \left.\left.+6\left(\eta_{\mathrm{disc}, 1, K}^{n, k, i}\right)^{2}+\left(\eta_{\mathrm{disc}, 2, K}^{n, k, i}\right)^{2}+\left|\eta_{\mathrm{disc}, 3, K}^{n, k, i}\right|+\left|\eta_{\mathrm{disc}, C, K}^{n, k, i}\right|\right](t) \mathrm{dt}\right\}^{\frac{1}{2}}, \\
\eta_{\mathrm{lin}}^{n, k, i}:= & 2^{\frac{1}{2}}\left\{\int_{I_{n}} \sum_{K \in \mathcal{T}_{h}}\left[6\left(\left(\eta_{\mathrm{lin}, 1, K}^{n, k, i}\right)^{2}+\left|\eta_{\operatorname{lin}, 3, K}^{n, k, i}\right|+\left|\eta_{\mathrm{lin}, C, K}^{n, k, i}\right|\right)+\left(\eta_{\mathrm{lin}, 2, K}^{n, k, i}\right)^{2}\right]\right\}^{\frac{1}{2}}, \\
\eta_{\mathrm{alg}}^{n, k, i}:= & \left\{6 \Delta t_{n} \sum_{K \in \mathcal{T}_{h}} \sum_{\alpha=1}^{2}\left(\eta_{\mathrm{alg}, K, \alpha}^{n, k, i}\right)^{2}\right\}^{\frac{1}{2}} .
\end{aligned}
$$


For $\beta \in\{$ disc, lin, alg $\}$, we introduce global-in-time estimators denoted by $\eta_{\beta}^{k, i}:=\left(\sum_{n=1}^{N_{\mathrm{t}}}\left(\eta_{\beta}^{n, k, i}\right)^{2}\right)^{\frac{1}{2}}$. Using Definition 5.1, we have:

Corollary 5.2. For $p \geq 1$, we have the following a posteriori error estimate distinguishing the error components:

$$
\left\|\boldsymbol{u}-\boldsymbol{u}_{h \tau}^{k, i} \mid\right\|_{\Omega, T} \leq \eta_{\mathrm{disc}}^{k, i}+\eta_{\mathrm{lin}}^{k, i}+\eta_{\mathrm{alg}}^{k, i}+\eta_{\text {init }} .
$$

Proof. Using (4.28) and (5.1), employing the Minkowski inequality, the Cauchy-Schwarz inequality, and the inequality $(A+B)^{\frac{1}{2}} \leq A^{\frac{1}{2}}+B^{\frac{1}{2}}$ for $A$ and $B \geq 0$ to gather the terms, we obtain the desired result.

\subsection{Adaptive inexact semismooth Newton algorithm}

We finally present our adaptive inexact semismooth Newton algorithm. Following the concept of [28, 46], it is designed to only perform the linearization and algebraic resolutions with minimal necessary precision, and thus to avoid unnecessary iterations, on the basis of the energy error a posteriori estimators of Definition 5.1. Let $\gamma_{\text {lin }}$ and $\gamma_{\text {alg }}$ be two positive parameters, typically of order 0.1 , representing the desired relative sizes of the algebraic and linearization errors. Supposing that $\eta_{\text {init }}$ is negligible, we propose:

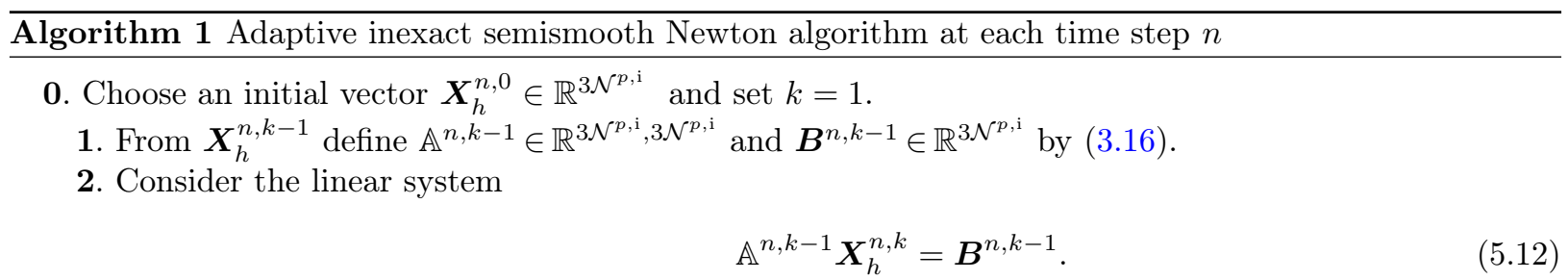

3. Set $\boldsymbol{X}_{h}^{n, k, 0}=\boldsymbol{X}_{h}^{n, k-1}$ (initial guess for the linear solver), set $i=0$.

4a. Perform $\nu \geq 1$ steps of a chosen linear solver for (5.12), starting from $\boldsymbol{X}_{h}^{n, k, i}$. Set $i=i+\nu$. This yields on step $i$ an approximation $\boldsymbol{X}_{h}^{n, k, i}$ satisfying

$$
\mathbb{A}^{n, k-1} \boldsymbol{X}_{h}^{n, k, i}=\boldsymbol{B}^{n, k-1}-\boldsymbol{R}^{n, k, i}
$$

4b. Compute the estimators of (5.10), (5.11) and check the stopping criterion for the linear solver in the form:

$$
\eta_{\mathrm{alg}}^{n, k, i} \leq \gamma_{\mathrm{alg}} \max \left\{\eta_{\mathrm{disc}}^{n, k, i}, \eta_{\text {lin }}^{n, k, i}\right\}
$$

If satisfied, set $\boldsymbol{X}_{h}^{n, k}=\boldsymbol{X}_{h}^{n, k, i}$. If not go back to $\mathbf{4 a}$.

5. Check the stopping criterion for the nonlinear solver in the form

$$
\eta_{\text {lin }}^{n, k, i} \leq \gamma_{\text {lin }} \eta_{\text {disc }}^{n, k, i}
$$

If satisfied, return $\boldsymbol{X}_{h}^{n}=\boldsymbol{X}_{h}^{n, k}$. If not, set $k=k+1$ and go back to 1 .

\section{$6 \quad$ Numerical experiments}

This section illustrates numerically our theoretical developments in the case of linear and quadratic finite elements $p=1$ and $p=2$. We first assume $p=1$ and that the semismooth Newton solver and the iterative algebraic solver have converged, i.e., we apply the "exact semismooth Newton" method as described in Section 3.7, using stopping criteria (3.18) and (3.19) with $\varepsilon_{\text {alg }}^{k}=10^{-11} \forall k \geq 1$ and $\varepsilon_{\text {lin }}=10^{-9}$. In this scenario, the semismooth Newton index $k$ and the linear iterative algebraic solver index $i$ will be discarded. We extend to the parabolic setting the test case given in [36] in which the domain $\Omega$ is given by the unit 
disk: $\Omega:=\{(r, \theta) \in[0,1] \times[0,2 \pi]\}$. We are interested in the shape of the numerical solution after several time steps and in the behavior of the estimators at convergence of the solvers given by Theorem 4.3.

Second, we will focus on our adaptive inexact semismooth Newton strategy given by Algorithm 1, based on Theorem 4.10, and Corollary 5.2. For this purpose, we will consider the geometry given in the first test case with different source terms. We will test our adaptive strategy with two semismooth Newton solvers: the Newton-min solver (see (3.13a)) with $p=1$ and $p=2$, and the Newton-Fischer-Burmeister solver (see (3.13c)) with $p=1$ only. We rely on the stopping criteria (5.13) and (5.14) with $\gamma_{\text {lin }}=\gamma_{\text {alg }}=10^{-3}$ for the adaptive inexact semismooth Newton strategy. The approaches with the exact Newton method and with the adaptive inexact Newton method are compared. The iterative algebraic solver that we employ at each semismooth Newton step $k \geq 1$ is GMRES (see [48]) with an ILU preconditioner with zero level fill-in.

For all the studies, the parameters $\mu_{1}$ and $\mu_{2}$ are set to 1 and the boundary condition for the first unknown $g$ is equal to 0.05 . We take a constant time step $\Delta t_{n}=\Delta t=0.001$ for all $1 \leq n \leq N_{\mathrm{t}}=300$, and the final time of simulation is $T=0.3$. The initial value is $\left(\boldsymbol{X}_{h}^{0}\right)^{T}=[g \mathbf{1}, \mathbf{0}]^{T} \in \mathbb{R}^{2 \mathcal{N}^{\bar{p}, \mathrm{i}}}$. For $p=1$, we consider a mesh containing approximately 21000 elements, and for $p=2$ a mesh with 4000 elements.

\subsection{Exact semismooth Newton method for $p=1$}

Following [36], we take a constant-in-time source term

$$
f_{1}(r, \theta, t):=\left\{\begin{array}{ll}
-10 g & \text { if } r \leq 1 / \sqrt{2}, \\
-8 g & \text { if } r \geq 1 / \sqrt{2},
\end{array} \quad f_{2}(r, \theta, t):= \begin{cases}-6 g & \text { if } r \leq 1 / \sqrt{2}, \\
-g \frac{1+8 r-18 r^{2}}{r} \frac{\sqrt{2}}{\sqrt{2}-1} & \text { if } r \geq 1 / \sqrt{2} .\end{cases}\right.
$$

In this case, $\left.f_{\alpha}\right|_{I_{n}}=\tilde{f}_{\alpha}^{n}$, so the data oscillation estimator $\eta_{\mathrm{osc}, \alpha}^{n}$ is zero.

In this section, we use linear finite elements $(p=1)$. Figure 1 displays the behavior of the numerical solution $\left(u_{1 h}^{n}, u_{2 h}^{n}, \lambda_{h}^{n}\right)$ at three instants $t=0.02, t=0.17$, and $t=0.3$. In the first situation, corresponding to the beginning of the simulation $t=0.02$ (first line of Figure 1), the complementarity constraint $u_{1 h}^{n}-u_{2 h}^{n}>0$ is satisfied, and the discrete Lagrange multiplier $\lambda_{h}^{n}$ vanishes. Next, at the time value $t=0.17, u_{1 h}^{n}$ and $u_{2 h}^{n}$ coincide in a subset of $\Omega$. Finally, at the end of the simulation $(t=0.3$, last line of Figure 1) the discrete Lagrange multiplier $\lambda_{h}^{n}$ is positive in the whole area $r \leq \frac{1}{\sqrt{2}}$, recovering the numerical result of the stationary case [36].

In Figure 2, the constraint estimator $\eta_{\mathrm{C}, K}^{n}(4.9)$ is plotted at $t=0.17$, and $t=0.3$ (it is 0 at $t=0.02$ ). It detects at each time step the elements where $u_{1 h}^{n}$ and $u_{2 h}^{n}$ become in contact (or detach one from another). We note that the constraint estimator $\eta_{\mathrm{C}, K}^{n}$ takes very small values.

Figure 3 displays the behavior of the flux estimator $\eta_{\mathrm{F}, K, 2}^{n}(4.7)$ and of the residual estimator $\eta_{\mathrm{R}, K, 2}^{n}(4.7)$ (see Theorem 4.3) associated to the second discrete unknown $u_{2 h}^{n}$ at the final simulation time $t=0.3$. We observe that the residual estimator $\eta_{\mathrm{R}, K, 2}^{n}$ is small with respect to the flux estimator $\eta_{\mathrm{F}, K, 2}^{n}$. Furthermore, in several elements $K \in \mathcal{T}_{h}$, the estimator $\eta_{\mathrm{F}, K, 2}^{n}$ is quite large which corresponds to zones where the finite element discretization error is important.

\subsection{Comparison of exact and adaptive inexact semismooth Newton algorithms}

The domain $\Omega$ is still the unit disk, but the data $f_{1}$ and $f_{2}$ are given by

$$
f_{1}(r, \theta, t):=\left\{\begin{array}{l}
-20 g \text { if } r \leq 1 / 5, \\
-50 g \text { if } 1 / 5 \leq r \leq 2 / 5, \\
+50 g \text { if } 2 / 5 \leq r \leq 3 / 5, \\
-50 g \text { if } 3 / 5 \leq r \leq 4 / 5, \\
+50 g \text { if } 4 / 5 \leq r \leq 1,
\end{array} \quad f_{2}(r, \theta, t):=\left\{\begin{array}{l}
+90 g \text { if } r \leq 1 / 5, \\
-40 g \text { if } 1 / 5 \leq r \leq 2 / 5, \\
+70 g \text { if } 2 / 5 \leq r \leq 3 / 5, \\
-30 g \text { if } 3 / 5 \leq r \leq 4 / 5, \\
+40 g \text { if } 4 / 5 \leq r \leq 1 .
\end{array}\right.\right.
$$

Here again $\eta_{\mathrm{osc}, \alpha}^{n}$ vanishes.

First of all, we display for several time steps the behavior of the numerical solution. Next, for a fixed time value, we represent the estimators as a function of the Newton iterations. Furthermore, for one selected Newton iteration, we also present the evolution of the various estimators as a function of the GMRES 

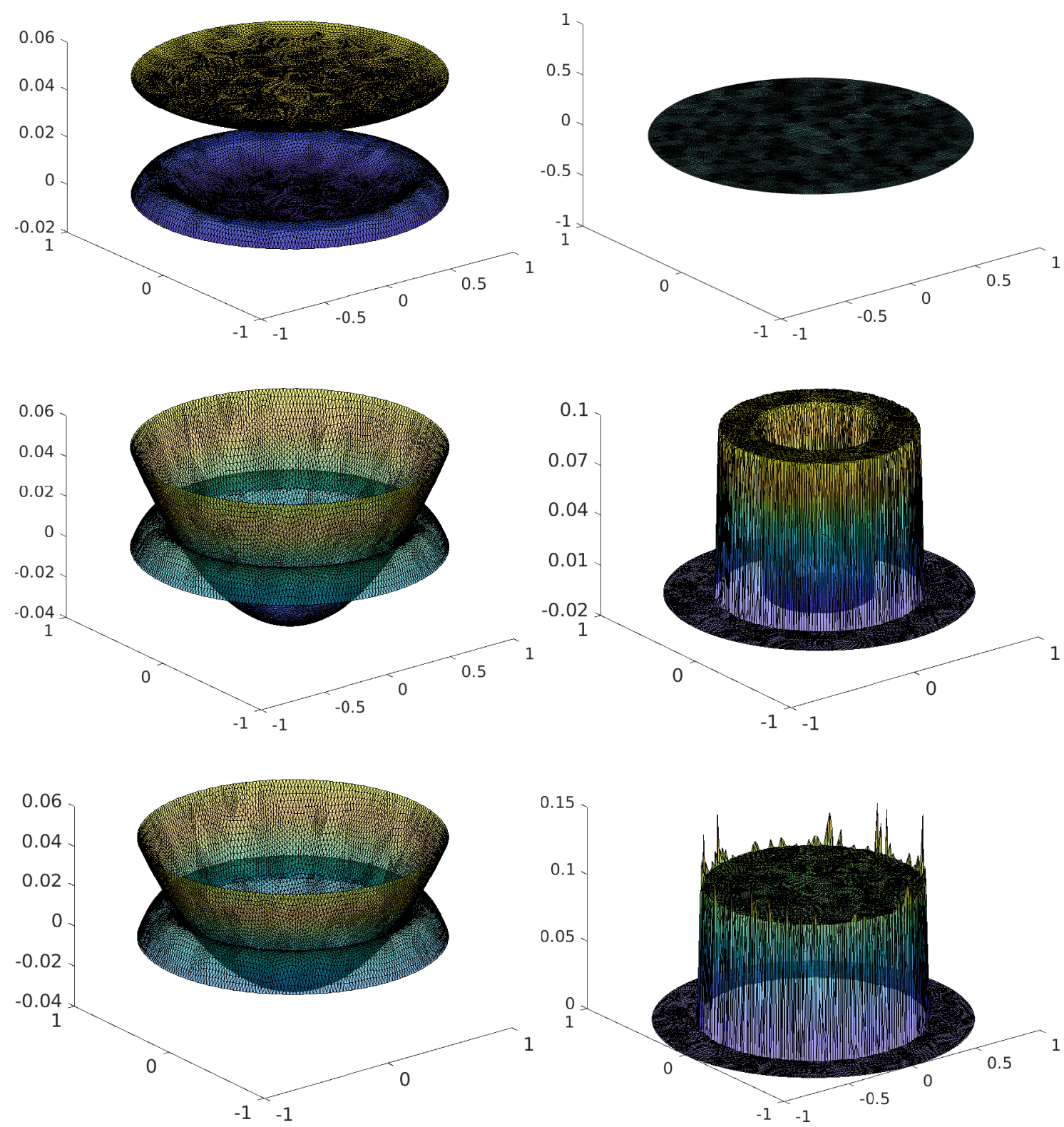

Figure 1: Numerical solution $u_{1 h}^{n}$ and $u_{2 h}^{n}$ (left column) and $\lambda_{h}^{n}$ (right column) at convergence for approximately 21000 elements with $p=1$. Time values from top to bottom: $t=0.02, t=0.17$, and $t=0.3$.

iterations. Finally, we test for each adaptive inexact semismooth Newton solver its overall performance and we compare the results with the classical exact resolution.

Figure 4 displays the numerical solution at three time values when the Newton-min solver and GMRES solver have converged. There are three different phases in the simulation: at first, there is no contact, see the first line of Figure 4. In the second period, the contact occurs in a disk around the center of the domain and we observe in the discrete Lagrange multiplier $\lambda_{h}^{n}$ a peak indicating the elements where $u_{1 h}^{n}$ and $u_{2 h}^{n}$ coincide. In the last period (last line of Figure 4), there exist two separate contact zones, a disk for $0 \leq r \leq 1 / 5$ and a ring for $2 / 5 \leq r \leq 3 / 5$. These contacts occur at $t \approx 0.011$ and $t \approx 0.060$; we will see below in Figures 6 and 9 (left) that more Newton-min iterations will be required at these transition periods. 

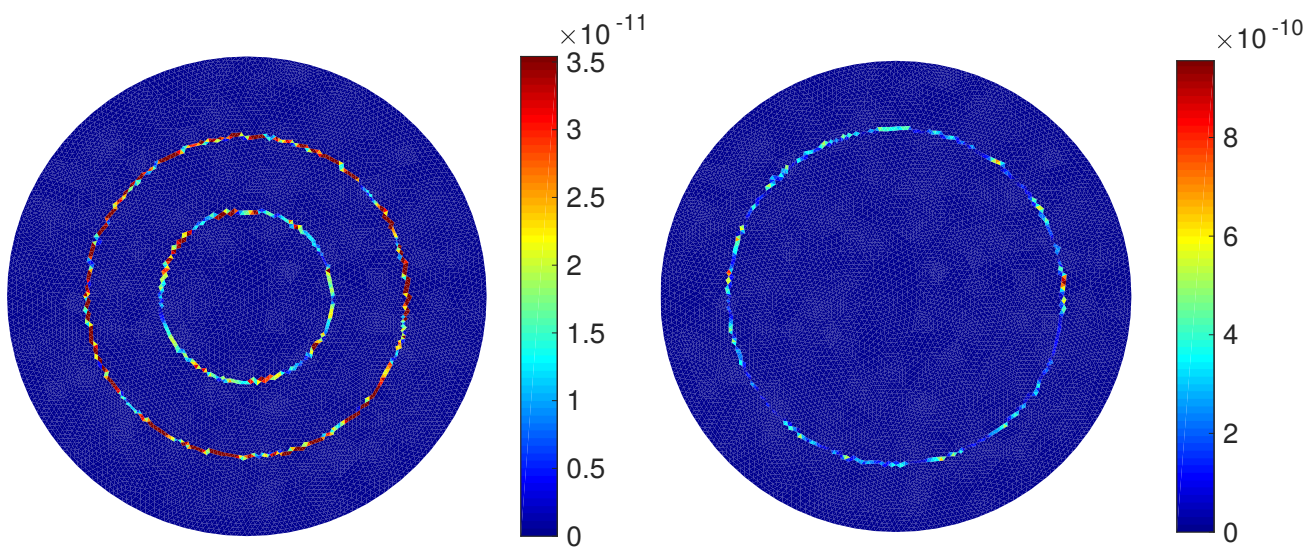

Figure 2: Constraint estimators $\eta_{\mathrm{C}, K}^{n}$ at convergence for approximately 21000 elements with $p=1$, at times $t=0.17$ (left) and $t=0.3$ (right).
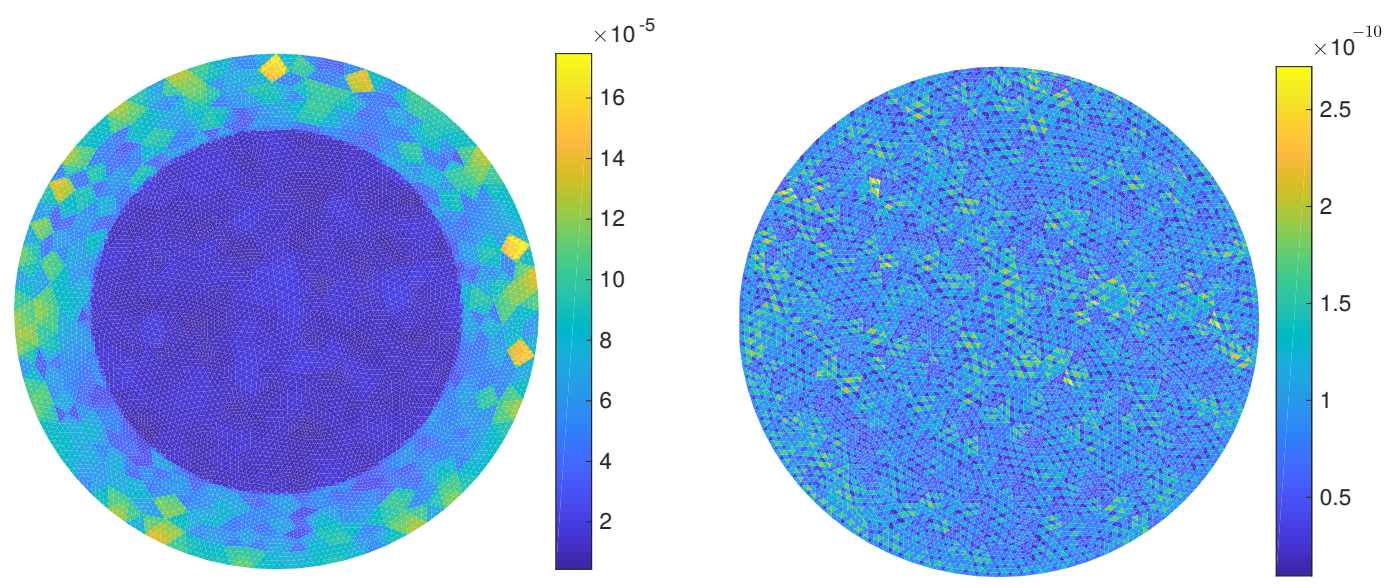

Figure 3: Estimators at convergence for approximately 21000 elements at $t=0.3$ with $p=1$. Left: flux estimator $\eta_{\mathrm{F}, K, 2}^{n}$. Right: residual estimator $\eta_{\mathrm{R}, K, 2}^{n}$.

\subsubsection{Newton-min linearization with $p=1$}

We present in this section some results for the adaptive inexact Newton-min Algorithm 1, taking the min $C$-function (3.13a), and using linear finite elements.

Figure 5 presents the evolution of the various estimators as a function of the Newton-min iterations (left), and as a function of the GMRES iterations at the first Newton-min step (right), both at the fixed time value $t=0.084$. From the left part of Figure 5 , we observe that the discretization estimator globally dominates and coincides with the total estimator (the two curves are roughly superimposed). The linearization estimator (blue curve) is small from the first Newton-min iteration (around $10^{-6}$ ) and next increases at the second iteration (around $10^{-3}$ ) and afterwards decreases rapidly to reach the value $10^{-11}$ at the third Newtonmin iteration. From the first Newton-min iteration, the discretization estimator (coinciding with the total estimator) stagnates which means that the other components of the error do not influence the behavior of the total error estimator. Then, the Newton-min algorithm performs unnecessary iterations and can be stopped at the first iteration. In right part of Figure 5, we test our adaptive inexact Newton-min strategy in terms of the GMRES iterations for the first Newton-min iteration. We observe that the discretization and linearization estimators roughly stagnate after few iterations. The algebraic estimator is large at the beginning of the iterations and influences the behavior of the total estimator but decreases rapidly to reach at $i=53$ the value $10^{-12}$. The adaptive inexact Newton-min algorithm stops the GMRES after $i=24$ iterations, when the total estimator almost coincides with the discretization estimator. Note that the curve of the algebraic estimator is here close to the curve of the norm $\left\|\boldsymbol{R}_{h}^{n, k, i}\right\|$ of the algebraic residual vector 

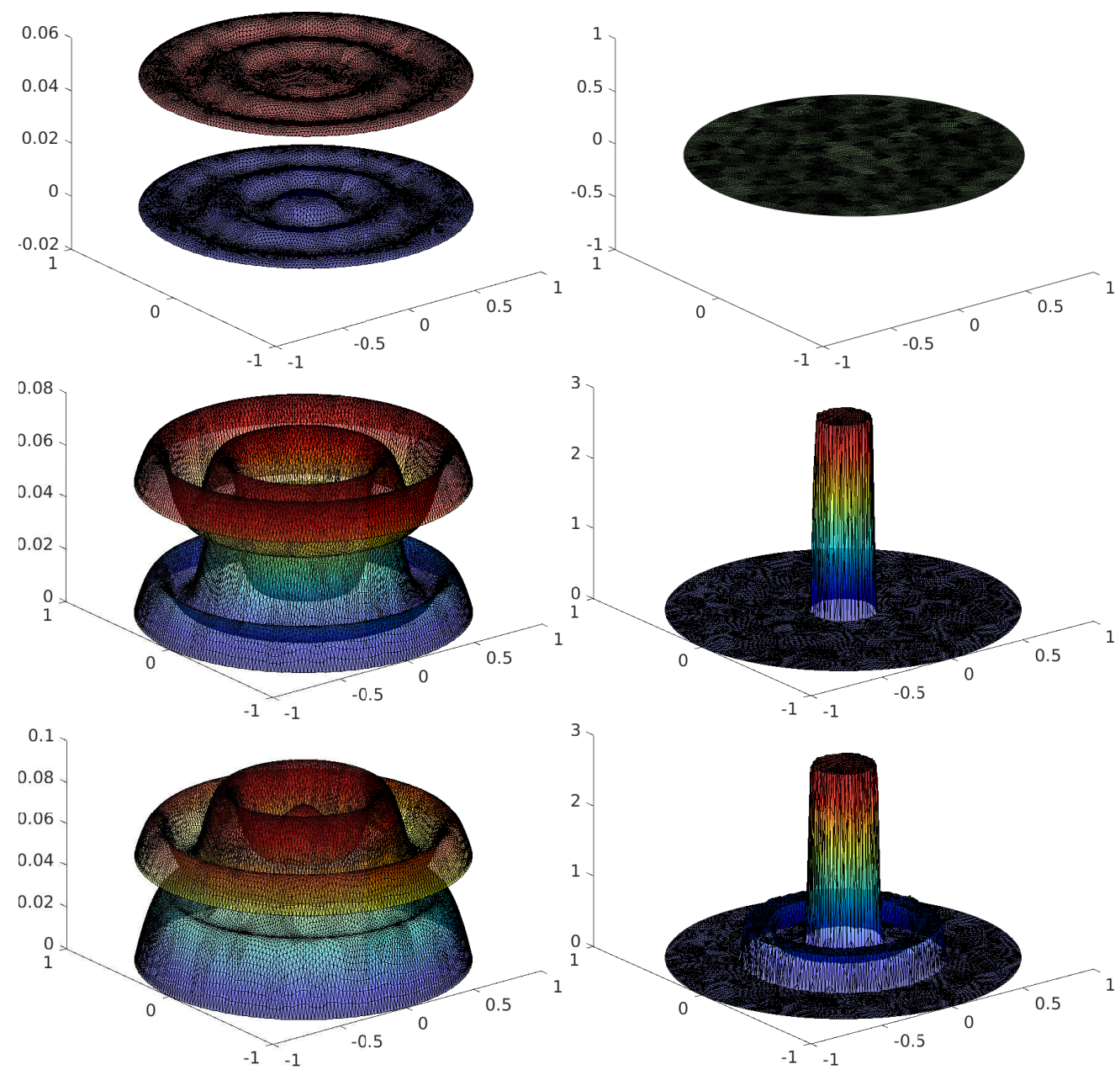

Figure 4: Numerical solutions $u_{1 h}^{n}$ and $u_{2 h}^{n}$ (left column) and $\lambda_{h}^{n}$ (right column) at convergence for approximately 21000 elements with $p=1$. Time values from top to bottom $t=0.001, t=0.03$, and $t=0.3$.
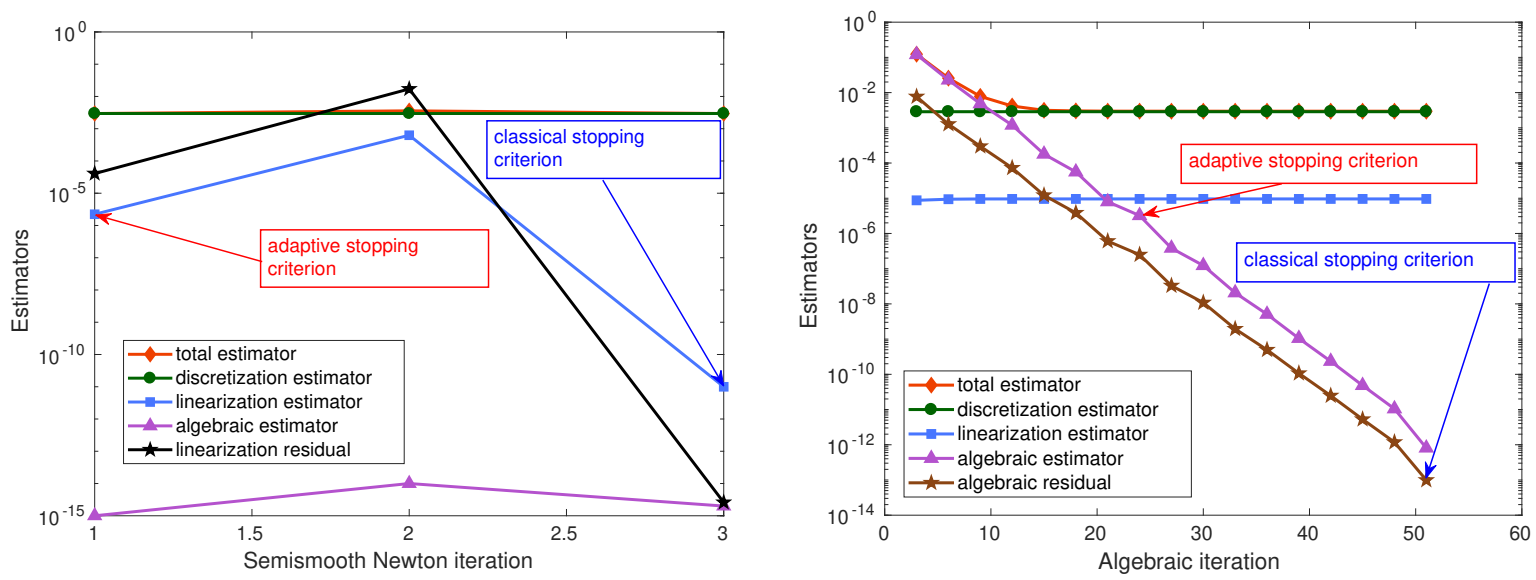

Figure 5: At $t=0.084$ with $p=1$. Left: estimators as a function of the Newton-min iterations. Right: estimators as a function of the GMRES iterations on 1st Newton-min iteration. 



Figure 6: Case $p=1$. Left: number of Newton-min iterations at each time step. Right: cumulated number of Newton-min iterations as a function of time.
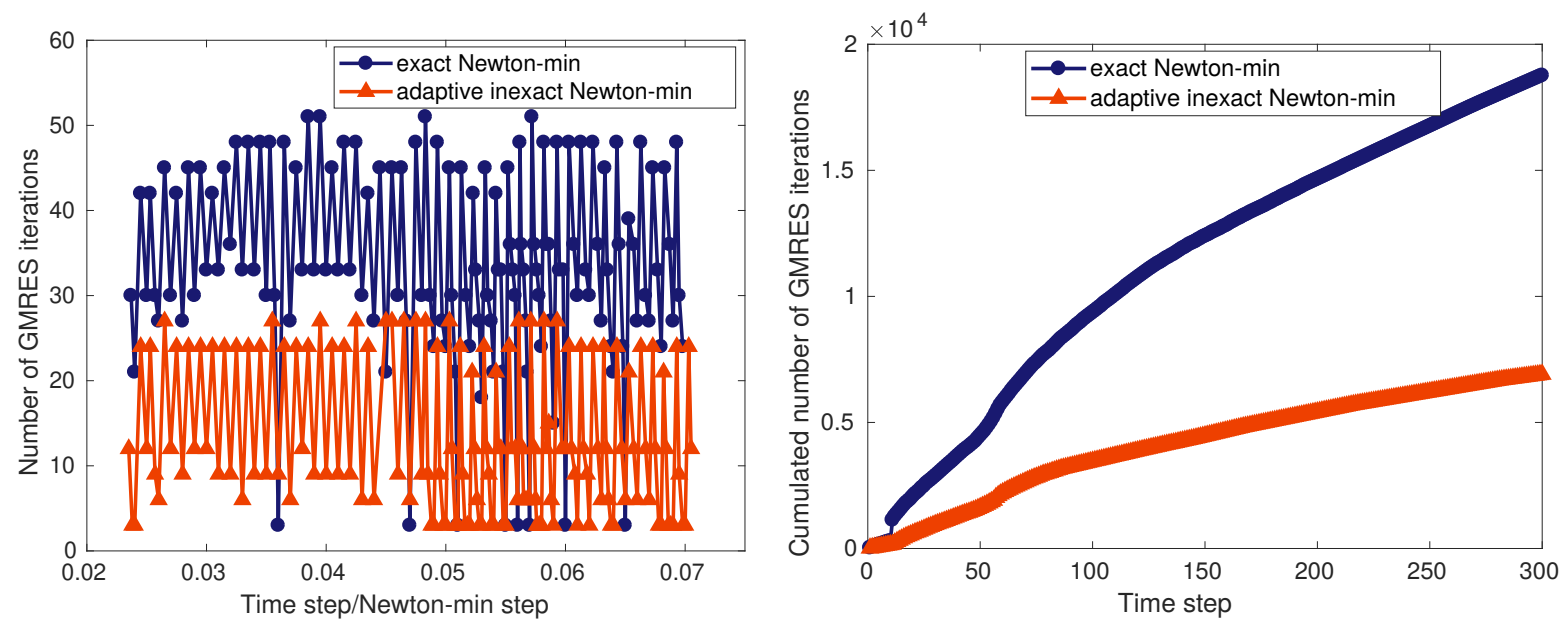

Figure 7: Case $p=1$. Left: number of GMRES iterations per time and Newton-min steps. Right: cumulated number of GMRES iterations as a function of time.

from (3.17).

Figure 6 provides the number of Newton-min iterations and the cumulated number of Newton-min iterations required to satisfy the given stopping criteria at each time step of the simulation. In particular, the first graph shows that for almost all time steps, our adaptive strategy is cheaper in terms of Newton-min iterations than the exact resolution. Observe that at some (rare) time steps (13 and 57 for instance), the adaptive approach requires more iterations than the classical resolution: it detects automatically when a few more iterations are necessary to preserve the accuracy. Interestingly, this occurs at times when $u_{1 h}^{n}$ and $u_{2 h}^{n}$ enter in contact. The second graph presents the cumulated number of Newton-min iterations as a function of the time step. We observe a substantial benefit for our adaptive inexact Newton-min approach as it saves at the end of the simulation roughly $50 \%$ of the iterations.

In Figure 7, left, we plot the number of GMRES iterations per time and Newton-min steps, between time steps 22 and 72 . We can observe that significantly fewer iterations are needed in the adaptive approach. We illustrate the overall performance of the two approaches in Figure 7, right, where we display the cumulated number of GMRES iterations for the two methods as a function of the time steps. The second graph shows that the adaptive inexact Newton-min algorithm requires approximately 7000 cumulated iterations to converge whereas the classical algorithm requires roughly 19000 iterations. Our adaptive algorithm thus saves many unnecessary iterations. 


\begin{tabular}{|l|l|l|l|l|l|l|}
\hline \multicolumn{2}{|l|}{$\gamma_{\text {alg }}=\gamma_{\text {lin }}=10^{-3}$} & $t_{n}=0.001$ & $t_{n}=0.011$ & $t_{n}=0.07$ & $t_{n}=0.15$ & $t_{n}=0.3$ \\
\hline$\| \mu_{1}^{\frac{1}{2}} \nabla\left(u_{1 h}^{n, \text { exact }}-u_{1 h}^{n, \text { adapt }}\right)$ & $\|_{\Omega}$ & $1.41 \times 10^{-7}$ & $1.81 \times 10^{-7}$ & $8.83 \times 10^{-8}$ & $2.03 \times 10^{-7}$ & $3.44 \times 10^{-7}$ \\
\hline$\left\|\mu_{2}^{\frac{1}{2}} \nabla\left(u_{2 h}^{n, \text { exact }}-u_{2 h}^{n, \text { adapt }}\right)\right\|_{\Omega}$ & $1.32 \times 10^{-7}$ & $1.63 \times 10^{-7}$ & $8.82 \times 10^{-8}$ & $1.71 \times 10^{-7}$ & $3.43 \times 10^{-7}$ \\
\hline$\left\|\lambda_{h}^{n, \text { exact }}-\lambda_{h}^{n, \text { adapt }}\right\|_{\Omega}$ & 0 & $2.77 \times 10^{-4}$ & $3.19 \times 10^{-4}$ & $4.25 \times 10^{-4}$ & $8.07 \times 10^{-8}$ \\
\hline
\end{tabular}

Table 1: Accuracy of the adaptive inexact Newton-min solution for several time values $(p=1)$.
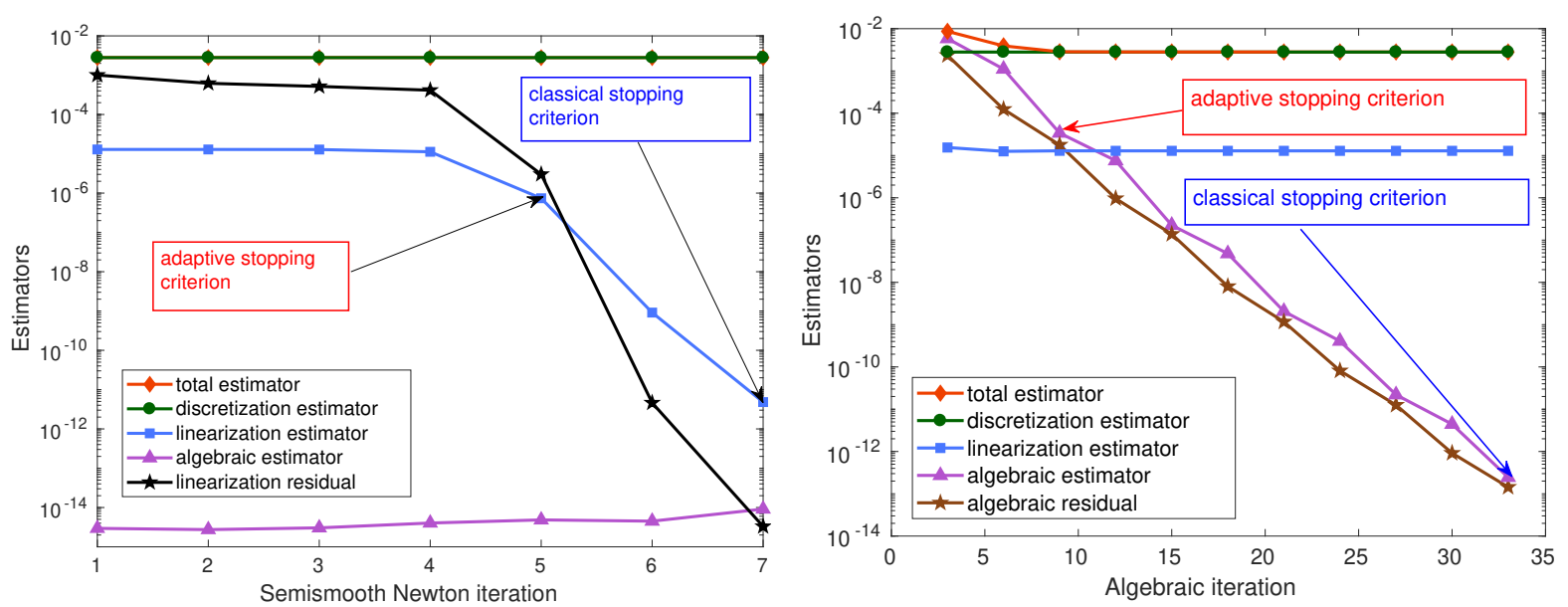

Figure 8: Case $p=1$. At $t=0.11$. Left: estimators as a function of the Newton-FB iterations. Right: estimators as a function of the GMRES iterations at the first Newton-FB step.

In Table 1, we give the global energy norm of the difference between the approximate solution given by the exact and by the adaptive inexact Newton-min algorithms. We observe that for several time values, the three numerical solutions are close to each other, which confirms that our adaptive strategy does not degrade the accuracy of the numerical solution.

\subsubsection{Newton-Fischer-Burmeister linearization with $p=1$}

In this section, we proceed as in Section 6.2.1, employing this time the $C$-function of Fischer-Burmeister (3.13c).

Figure 8 represents the evolution of the various estimators as a function of the Newton-Fischer-Burmeister (denoted Newton-FB in the sequel) iterations (left), and as a function of the GMRES iterations at the first Newton-FB step (right), both at the time value $t=0.011$. From the left plot, we observe that the discretization estimator globally dominates and almost coincides with the total estimator (the two curves are roughly superimposed). The linearization estimator is small and decreases rapidly after $k=5$ steps (adaptive stopping criterion) to reach the value of $10^{-11}$ at $k=11$ (classical stopping criterion). Taking $\gamma_{\text {lin }}=10^{-2}$ instead of $\gamma_{\text {lin }}=10^{-3}$ in (5.14) will reduce the number of Newton-FB iterations at this instant to merely 4 iterates. In the right plot, we take the first Newton-FB iteration and we observe that the discretization and the linearization estimators stagnate from the beginning of the iterations, while the algebraic estimator is dominant at the beginning. The adaptive inexact Newton-FB algorithm stops the GMRES iterations at $i=9$, whereas the classical criterion stops at $i=33$. Note that, like in the Newton-min case, the behavior of the algebraic estimator follows closely the one of the algebraic residual.

Figure 9 focuses on the number of Newton-FB iterations required to satisfy the various stopping criteria at each time step. We observe from the first figure that the adaptive strategy (red curve) is economic in comparison with the classical resolution especially from $t=0.1$ onwards, where the adaptive algorithm requires 1 Newton-FB iteration at each time step. Furthermore, the right plot depicts the overall performance in terms of Newton-FB iterations. With no surprise, the adaptive resolution requires at the end of the simulation much fewer semismooth Newton iterations (approximately 700 for the adaptive algorithms 

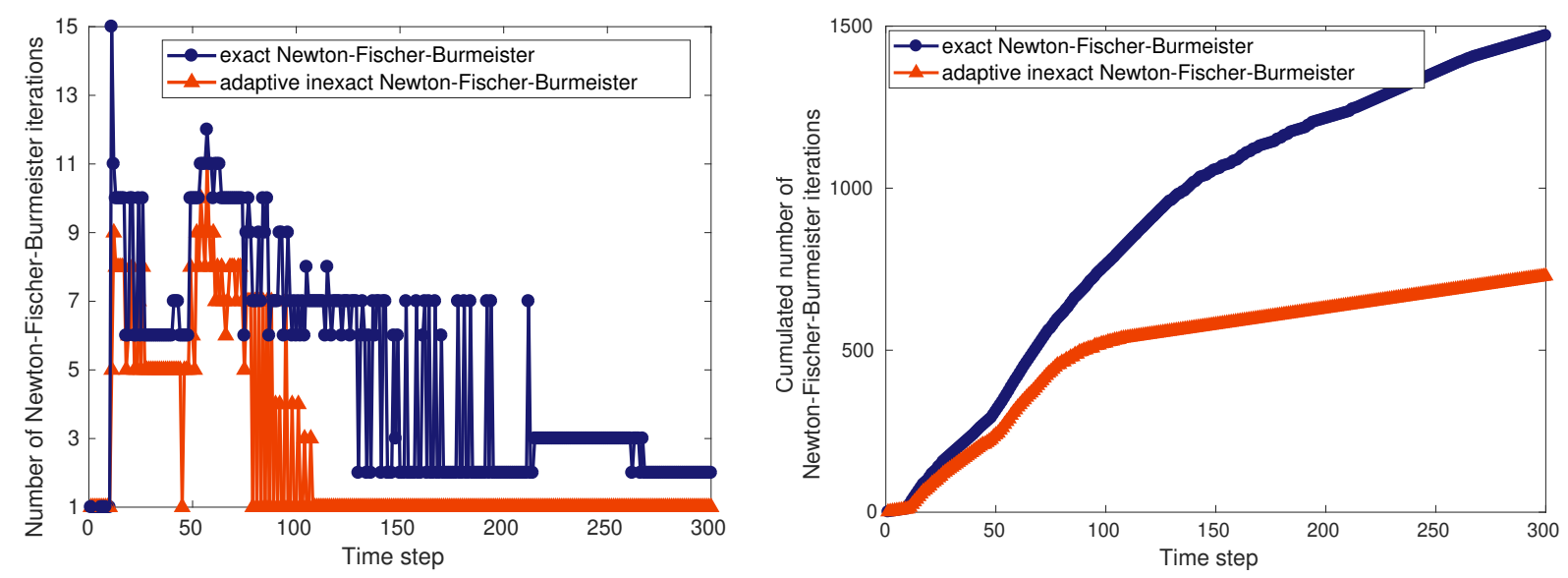

Figure 9: Case $p=1$. Left: number of Newton-FB iterations at each time step. Right: cumulated number of Newton-FB iterations as a function of time.
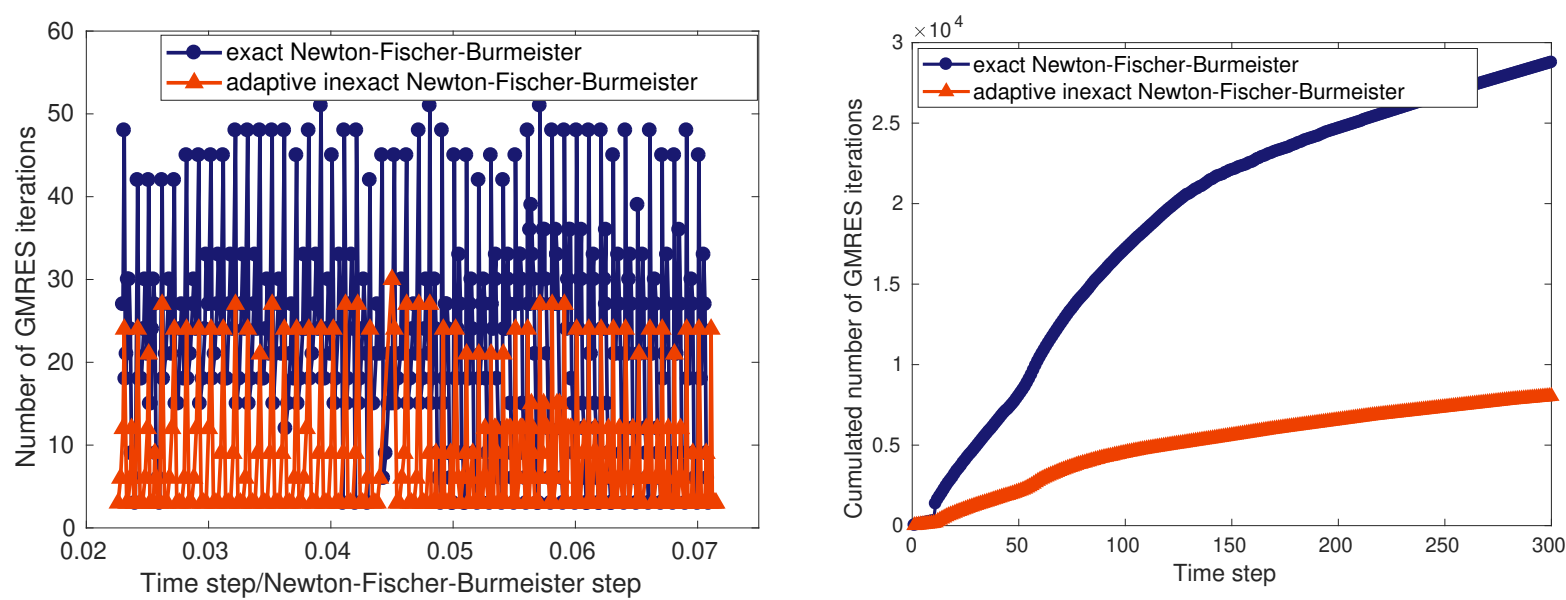

Figure 10: Case $p=1$. Left: number of GMRES iterations per time and Newton-FB step. Right: cumulated number of GMRES iterations per time step.

and 1500 for the classical resolution). Thus, our adaptive semismooth approach reduces by $50 \%$ the number of Newton-FB iterations.

Figure 10 illustrates the overall performance of the two approaches. We display the number of GMRES iterations for each linear system solved as a function of time/Newton-FB step between $t=0.014$ and $t=0.057$ (left) and the cumulated number of GMRES iterations as a function of time step (right). We see that our adaptive strategy is very economic in terms of the total algebraic iterations, as it requires at the end of the simulation approximately 7000 iterations, whereas the classical resolution requires roughly 27000 iterations. We present in Table 2 the energy norm of the difference between the exact solution given

\begin{tabular}{|c|c|c|c|c|c|c|}
\hline$\gamma_{\text {alg }}=\gamma_{\text {lin }}=10^{-3}$ & $t_{n}=0.001$ & $t_{n}=0.011$ & $t_{n}=0.07$ & $t_{n}=0.15$ & $t_{n}=0.3$ \\
\hline$\left\|\mu_{1}^{\frac{1}{2}} \nabla\left(u_{1 h}^{n, \text { exact }}-u_{1 h}^{n, \text { adapt }}\right)\right\|_{\Omega}$ & $9.9 \times 10^{-6}$ & $1.7 \times 10^{-5}$ & $5.8 \times 10^{-5}$ & $7.7 \times 10^{-5}$ & $2.1 \times 10^{-3}$ \\
\hline$\| \mu_{2}^{\frac{1}{2}} \nabla\left(u_{2 h}^{n, \text { exact }}-u_{2 h}^{n, \text { adapt }}\right)$ & $\|_{\Omega}$ & $5.5 \times 10^{-6}$ & $2.1 \times 10^{-5}$ & $7.1 \times 10^{-5}$ & $1.8 \times 10^{-4}$ & $2.1 \times 10^{-3}$ \\
\hline \multicolumn{2}{||}{$\mid \lambda_{h}^{n, \text { exact }}-\lambda_{h}^{n, \text { adapt }} \|_{\Omega}$} & 0 & $7.9 \times 10^{-3}$ & $3.3 \times 10^{-4}$ & $2.3 \times 10^{-2}$ & $2.2 \times 10^{-7}$ \\
\hline
\end{tabular}

Table 2: Accuracy of the adaptive inexact Newton-FB solution for several time values $(p=1)$. 

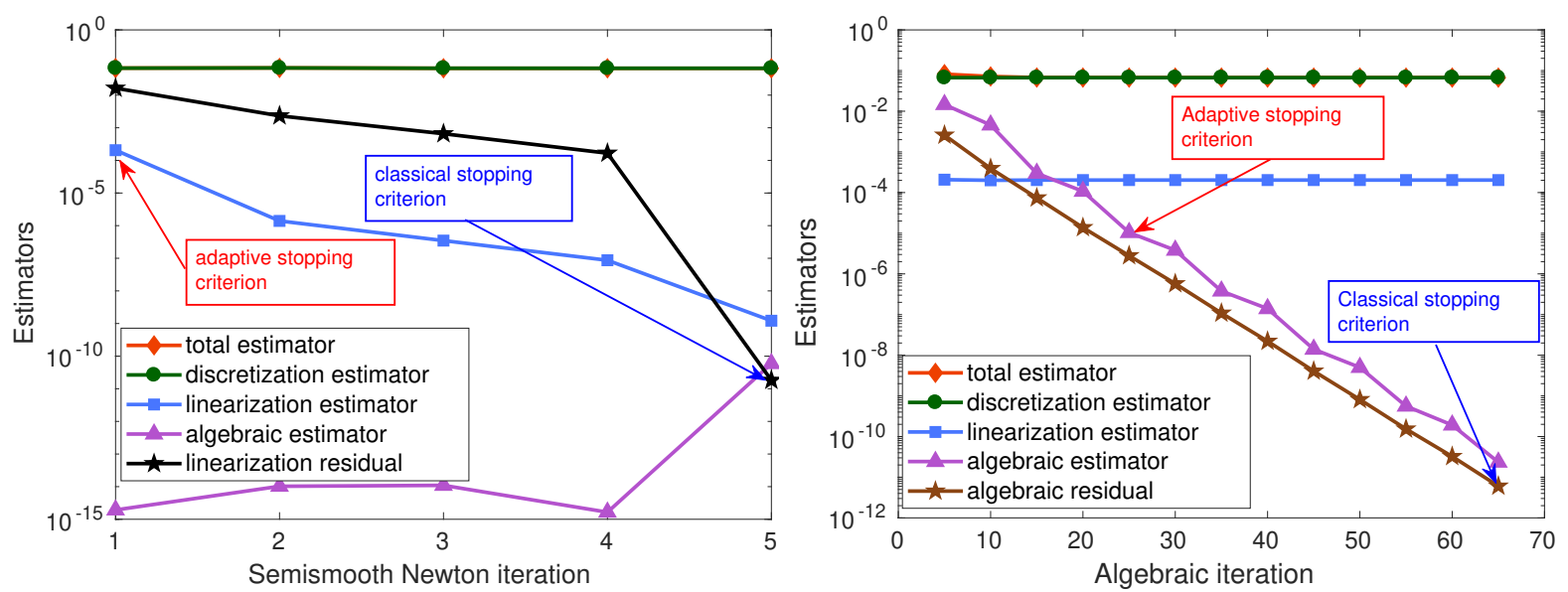

Figure 11: Case $p=2$. At $t=0.14$. Left: estimators as a function of Newton-min iterations. Right: estimators as a function of GMRES iterations at the first Newton-min step.

by the classical Newton-FB algorithm and the adaptive inexact one for several time values. In particular, it measures the accuracy and precision of our adaptive strategy. We observe that each numerical unknown obtained by the adaptive strategy is close to the unknown given by the classical resolution. Thus, our adaptive algorithm saves many iterations and does not deteriorate the numerical solution.

\subsubsection{Newton-min linearization with $p=2$}

In this section, we present results for the adaptive inexact Newton-min algorithm with quadratic finite elements $(p=2)$. Figures 11,12 , and 13 are respectively the counterparts of Figures 5,6 , and 7 . The comments made in Section 6.2.1 apply globally to this case.

In Figures 12 and 13 (right graphs), we plotted the cumulated number of Newton-min iterations and of GMRES iterations for both linear and quadratic finite elements, using the same mesh with 4000 elements. Note that there are 4 times more degrees of freedom when $p=2$ than with $p=1$. In the case $p=2$, the exact Newton-min requires 912 semismooth iterations, whereas the adaptive inexact Newton-min requires only 585 semismooth iterations, see Figure 12 . The economy is more impressive in terms of cumulated GMRES iterations, see Figure 13. In the case $p=2$, the adaptive inexact algorithm is roughly 3 times cheaper in terms of number of cumulated GMRES iterates than the exact version (8760 iterates instead of 27085). When passing from $p=1$ to $p=2$, we note that the rate of increase of the number of iterations is smaller for the adaptive inexact case than for the exact Newton-min case. Indeed, the number of cumulated GMRES increases by a factor of 3.5 in the exact case, and only by a factor 2.8 in the adaptive case.

\subsection{Comments on implementation and cost}

In terms of CPU, the adaptive inexact semismooth strategy presented here requires an overhead to compute the estimators. In particular, the computation of the algebraic error flux reconstruction based on a multilevel strategy can be a little long. However, all the estimators enable a completely parallel implementation, and we believe that the cost is worth paying for, as 1) the number of iterations (both semismooth Newton and algebraic) is drastically reduced. It seems that the more expensive the simulation (compare $p=1$ and $p=2$ ), the greater the gain. And 2) the guarantee on the total error is a real advantage, as one can have a confidence in the result.

The computation of the parabolic estimators in the general case $(p \geq 2$, when the linearization and algebraic solvers have not converged, see Definitions 4.9 and 5.1), requires the evaluation of positive and negative parts of $\lambda_{h}^{n, k, i}$, as well as the evaluation of $\tilde{\lambda}_{h}^{n, k, i, \operatorname{pos}}$ and $\tilde{\lambda}_{h}^{n, k, i, \text { neg }}$. The latter terms are easy to compute, as it suffices to restrict the vector $\boldsymbol{X}_{3 h}^{n, k, i}$ to its positive and negative parts (recall that $\lambda_{h}$ is decomposed in the dual basis to the Lagrange basis function). The former terms however do not take a 

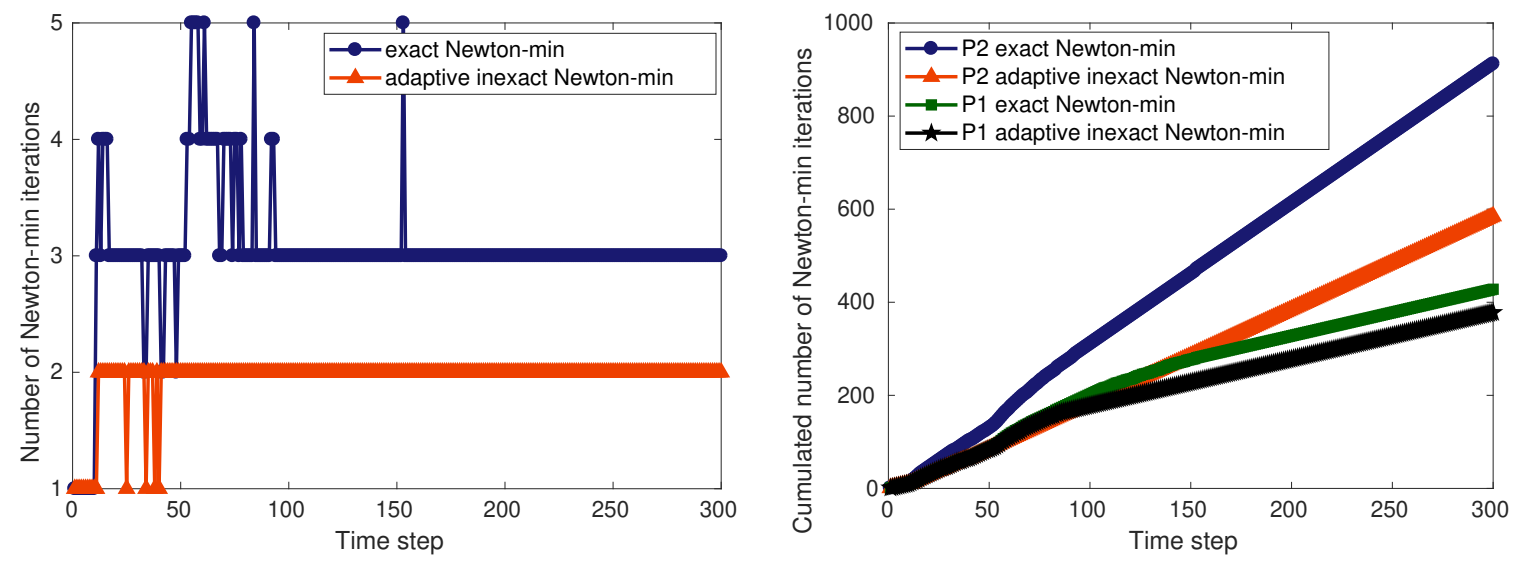

Figure 12: Case $p=2$. Left: number of Newton-min iterations at each time step. Right: cumulated number of Newton-min iterations as a function of time, comparison $p=1, p=2$.


Figure 13: Case $p=2$. Left: number of GMRES iterations per time and Newton-min step between $t=0.008$ and $t=0.065$. Right: cumulated number of GMRES iterations per time step, comparison $p=1, p=2$.

polynomial form on the mesh $\mathcal{T}_{h}$ and are more expensive to compute. To integrate approximately the terms $\lambda_{h}^{n, k, i, \text { pos }}$ and $\lambda_{h}^{n, k, i, \text { neg }}$ (see $\eta_{\mathrm{C}, K}^{n, k, i, \text { pos }}$ for instance), we typically separate the positive and negative parts of $\lambda_{h}^{n, k, i}$ in the quadrature points.

\section{Conclusion}

In this work, we focused on deriving a posteriori error estimates for a model parabolic variational inequality. We employed the conforming $\mathbb{P}_{p}$ finite element method for the discretization in space and the backward Euler scheme for the discretization in time. We designed a posteriori error estimates when $p=1$ valid at convergence of the semismooth Newton solver and of the iterative algebraic solver. In this case, we estimate both energy and time derivative errors. Next, we extended the study to all polynomial degrees $p \geq 1$ and for each semismooth Newton step $k \geq 1$ and each iterative linear algebraic solver step $i \geq 0$. Here, we only estimate the energy error. Based on the separation of the components of the errors, we finally propose an adaptive inexact semismooth Newton algorithm The main idea is to stop the two involved iterative solvers at a suitable moment decided adaptively. We have presented numerical experiments for two inexact semismooth Newton solvers for $p=1$ and $p=2$, and we showed that our adaptive inexact semismooth strategy saves many iterations while preserving the accuracy of the numerical solution. 


\section{References}

[1] R. Glowinski, Numerical methods for nonlinear variational problems, Scientific Computation, SpringerVerlag, Berlin, 2008, reprint of the 1984 original.

[2] J.-L. Lions, Quelques méthodes de résolution des problèmes aux limites non linéaires, Dunod; GauthierVillars, Paris, 1969.

[3] Y. Achdou, F. Hecht, D. Pommier, A posteriori error estimates for parabolic variational inequalities, J. Sci. Comput. 37 (3) (2008) 336-366. doi:10.1007/s10915-008-9215-7. URL https://doi.org/10.1007/s10915-008-9215-7

[4] T. Gudi, P. Majumder, Convergence analysis of finite element method for a parabolic obstacle problem, J. Comput. Appl. Math. 357 (2019) 85-102. doi:10.1016/j.cam.2019.02.026. URL https://doi.org/10.1016/j.cam.2019.02.026

[5] P. Jaillet, D. Lamberton, B. Lapeyre, Variational inequalities and the pricing of american options, ACTA APPL. MATH 21 (1990) 263-289.

[6] P. Wilmott, J. Dewynne, S. Howison, Option Pricing: Mathematical Models and Computation, Oxford Financial, 1998. URL https://books . google.fr/books?id=cGuGcgAACAAJ

[7] J.-F. Rodrigues, The variational inequality approach to the one-phase Stefan problem, Acta Appl. Math. 8 (1) (1987) 1-35. doi:10.1007/BF00046685. URL https://doi.org/10.1007/BF00046685

[8] A. Lauser, C. Hager, R. Helmig, B. Wohlmuth, A new approach for phase transitions in miscible multi-phase flow in porous media, Advances in Water Resources 68 (2011) 957-966.

[9] J. Steinbach, A variational inequality approach to free boundary problems with applications in mould filling, Vol. 136, Birkhäuser, 2012.

[10] I. Ben Gharbia, J. Dabaghi, V. Martin, M. Vohralík, A posteriori error estimates for a compositional two-phase flow with nonlinear complementarity constraints, Comput. Geosci. doi:10.1007/ s10596-019-09909-5.

URL https://hal.archives-ouvertes.fr/hal-01919067

[11] S. J. Wright, Primal-dual interior-point methods, Society for Industrial and Applied Mathematics (SIAM), Philadelphia, PA, 1997. doi:10.1137/1.9781611971453. URL http://dx.doi.org/10.1137/1.9781611971453

[12] C. Kanzow, An active set-type Newton method for constrained nonlinear systems, in: Complementarity: applications, algorithms and extensions (Madison, WI, 1999), Vol. 50 of Appl. Optim., Kluwer Acad. Publ., Dordrecht, 2001, pp. 179-200. doi:10.1007/978-1-4757-3279-5_9.

URL http://dx.doi.org/10.1007/978-1-4757-3279-5_9

[13] T. De Luca, F. Facchinei, C. Kanzow, A semismooth equation approach to the solution of nonlinear complementarity problems, Math. Programming 75 (3, Ser. A) (1996) 407-439.

URL https://doi.org/10.1016/S0025-5610(96)00028-7

[14] M. Hintermüller, K. Ito, K. Kunisch, The primal-dual active set strategy as a semismooth Newton method, SIAM J. Optim. 13 (3) (2002) 865-888 (2003). doi:10.1137/S1052623401383558.

URL http://dx.doi.org/10.1137/S1052623401383558

[15] K. Ito, K. Kunisch, Semi-smooth Newton methods for variational inequalities of the first kind, ESAIM Math. Model. Numer. Anal. 37 (1) (2003) 41-62. doi:10.1051/m2an:2003021.

URL http://www. numdam.org/item/M2AN_2003__37_1_41_0

[16] F. Facchinei, J.-S. Pang, Finite-dimensional variational inequalities and complementarity problems. Vol. I, Springer Series in Operations Research, Springer-Verlag, New York, 2003. 
[17] F. Facchinei, J.-S. Pang, Finite-dimensional variational inequalities and complementarity problems. Vol. II, Springer Series in Operations Research, Springer-Verlag, New York, 2003.

[18] G. Stadler, Semismooth Newton and augmented Lagrangian methods for a simplified friction problem, SIAM J. Optim. 15 (2004) 39-62. doi:10.1137/S1052623403420833.

[19] S. Zhang, Y. Yan, R. Ran, Path-following and semismooth Newton methods for the variational inequality arising from two membranes problem, J. Inequal. Appl. 2019 (1). doi:10.1186/ s13660-019-1955-4.

URL https://doi.org/10.1186/s13660-019-1955-4

[20] J. F. Bonnans, J. C. Gilbert, C. Lemaréchal, C. A. Sagastizábal, Numerical optimization, 2nd Edition, Universitext, Springer-Verlag, Berlin, 2006, theoretical and practical aspects.

[21] M. Ainsworth, J. T. Oden, A posteriori error estimation in finite element analysis, Pure and Applied Mathematics (New York), Wiley-Interscience [John Wiley \& Sons], New York, 2000. doi:10.1002/ 9781118032824.

URL http://dx.doi.org/10.1002/9781118032824

[22] R. Verfürth, A posteriori error estimation techniques for finite element methods, Numerical Mathematics and Scientific Computation, Oxford University Press, Oxford, 2013. doi:10.1093/acprof: oso/9780199679423.001.0001.

URL http://dx.doi.org/10.1093/acprof : oso/9780199679423.001.0001

[23] M. Ainsworth, J. T. Oden, C.-Y. Lee, Local a posteriori error estimators for variational inequalities, Numer. Methods Partial Differential Equations 9 (1) (1993) 23-33. doi:10.1002/num.1690090104. URL https://doi.org/10.1002/num.1690090104

[24] Z. Chen, R. H. Nochetto, Residual type a posteriori error estimates for elliptic obstacle problems, Numer. Math. 84 (4) (2000) 527-548. doi:10.1007/s002110050009.

URL https://doi.org/10.1007/s002110050009

[25] A. Veeser, Efficient and reliable a posteriori error estimators for elliptic obstacle problems, SIAM J. Numer. Anal. 39 (1) (2001) 146-167. doi:10.1137/S0036142900370812.

URL https://doi.org/10.1137/S0036142900370812

[26] T. Gudi, K. Porwal, A posteriori error control of discontinuous Galerkin methods for elliptic obstacle problems, Math. Comp. 83 (286) (2014) 579-602. doi:10.1090/S0025-5718-2013-02728-7. URL https://doi.org/10.1090/S0025-5718-2013-02728-7

[27] M. Bürg, A. Schröder, A posteriori error control of $h p$-finite elements for variational inequalities of the first and second kind, Comput. Math. Appl. 70 (12) (2015) 2783-2802. doi:10.1016/j.camwa.2015. 08.031.

URL https://doi.org/10.1016/j.camwa.2015.08.031

[28] J. Dabaghi, V. Martin, M. Vohralík, Adaptive inexact semismooth Newton methods for the contact problem between two membranes, HAL Preprint 01666845, submitted for publication (2020). URL https://hal.inria.fr/hal-01666845

[29] R. Verfürth, A posteriori error estimates for nonlinear problems: $L^{r}\left(0, T ; W^{1, \rho}(\Omega)\right)$-error estimates for finite element discretizations of parabolic equations, Numer. Methods Partial Differential Equations 14 (4) (1998) 487-518. doi:10.1002/(SICI) 1098-2426 (199807) 14:4<487: : AID-NUM4>3. 0. C0;2-G. URL https://doi.org/10.1002/(SICI) 1098-2426(199807) 14:4<487 : :AID-NUM4>3.0. CO;2-G

[30] A. Bergam, C. Bernardi, Z. Mghazli, A posteriori analysis of the finite element discretization of some parabolic equations, Math. Comp. 74 (251) (2005) 1117-1138. doi :10.1090/S0025-5718-04-01697-7. URL https://doi.org/10.1090/S0025-5718-04-01697-7 
[31] A. Ern, I. Smears, M. Vohralík, Guaranteed, locally space-time efficient, and polynomial-degree robust a posteriori error estimates for high-order discretizations of parabolic problems, SIAM J. Numer. Anal. 55 (6) (2017) 2811-2834. doi:10.1137/16M1097626.

URL https://doi.org/10.1137/16M1097626

[32] A. Ern, I. Smears, M. Vohralík, Equilibrated flux a posteriori error estimates in $L^{2}\left(H^{1}\right)$-norms for high-order discretizations of parabolic problems, IMA J. Numer. Anal. 39 (3) (2019) 1158-1179.

URL https://doi.org/10.1093/imanum/dry035

[33] K.-S. Moon, R. H. Nochetto, T. V. Petersdorff, C.-S. Zhang, A posteriori error analysis for parabolic variational inequalities, ESAIM Math. Model. Numer. Anal. 41 (3) (2007) 485-511. doi:10.1051/ $\mathrm{m} 2 \mathrm{an}: 2007029$.

URL http://www. numdam.org/item/M2AN_2007_-_41_3_485_0

[34] H. Gimperlein, J. Stocek, Space-time adaptive finite elements for nonlocal parabolic variational inequalities, Comput. Methods Appl. Mech. Engrg. 352 (2019) 137-171. doi:10.1016/j.cma.2019.04.019. URL https://doi.org/10.1016/j.cma.2019.04.019

[35] F. Ben Belgacem, C. Bernardi, A. Blouza, M. Vohralík, A finite element discretization of the contact between two membranes, M2AN Math. Model. Numer. Anal. 43 (1) (2008) 33-52. doi:10.1051/m2an/ 2008041.

URL http://dx.doi.org/10.1051/m2an/2008041

[36] F. Ben Belgacem, C. Bernardi, A. Blouza, M. Vohralík, On the unilateral contact between membranes. Part 2: a posteriori analysis and numerical experiments, IMA J. Numer. Anal. 32 (3) (2012) 1147-1172. doi:10.1093/imanum/drr003.

URL http://dx.doi.org/10.1093/imanum/drr003

[37] F. Ben Belgacem, C. Bernardi, A. Blouza, M. Vohralík, On the unilateral contact between membranes. Part 1: Finite element discretization and mixed reformulation, Math. Model. Nat. Phenom. 4 (1) (2009) 21-43. doi:10.1051/mmnp/20094102.

URL http://dx.doi.org/10.1051/mmnp/20094102

[38] J. Dabaghi, A posteriori error estimates for variational inequalities: application to a two-phase flow in porous media, Ph.D. thesis, Sorbonne Université (Jun. 2019).

URL https://hal.archives-ouvertes.fr/tel-02151951

[39] H. Brezis, Functional analysis, Sobolev spaces and partial differential equations, Universitext, Springer, New York, 2011.

[40] M. Ulbrich, Semismooth Newton methods for variational inequalities and constrained optimization problems in function spaces, Vol. 11 of MOS-SIAM Series on Optimization, Society for Industrial and Applied Mathematics (SIAM), Philadelphia, PA; Mathematical Optimization Society, Philadelphia, PA, 2011. doi:10.1137/1.9781611970692.

URL https://doi.org/10.1137/1.9781611970692

[41] S. C. Eisenstat, H. F. Walker, Globally convergent inexact Newton methods, SIAM J. Optim. 4 (2) (1994) 393-422. doi:10.1137/0804022.

URL http://dx.doi.org/10.1137/0804022

[42] C. T. Kelley, Iterative methods for linear and nonlinear equations, Vol. 16 of Frontiers in Applied Mathematics, Society for Industrial and Applied Mathematics (SIAM), Philadelphia, PA, 1995.

URL https://doi.org/10.1137/1.9781611970944

[43] P. Destuynder, B. Métivet, Explicit error bounds in a conforming finite element method, Math. Comp. 68 (228) (1999) 1379-1396. doi:10.1090/S0025-5718-99-01093-5.

URL http://dx.doi.org/10.1090/S0025-5718-99-01093-5 
[44] D. Braess, J. Schöberl, Equilibrated residual error estimator for edge elements, Math. Comp. 77 (262) (2008) 651-672. doi:10.1090/S0025-5718-07-02080-7.

URL http://dx.doi.org/10.1090/S0025-5718-07-02080-7

[45] J. Papež, U. Rüde, M. Vohralík, B. Wohlmuth, Sharp algebraic and total a posteriori error bounds for $h$ and $p$ finite elements via a multilevel approach. Recovering mass balance in any situation, HAL Preprint 01662944, submitted for publication (2019).

URL https://hal.inria.fr/hal-01662944/

[46] A. Ern, M. Vohralík, Adaptive inexact Newton methods with a posteriori stopping criteria for nonlinear diffusion PDEs, SIAM J. Sci. Comput. 35 (4) (2013) A1761-A1791. doi:10.1137/120896918.

URL http://dx.doi.org/10.1137/120896918

[47] P.-A. Raviart, J.-M. Thomas, A mixed finite element method for 2nd order elliptic problems, in: Mathematical aspects of finite element methods (Proc. Conf., Consiglio Naz. delle Ricerche (C.N.R.), Rome, 1975, Springer, Berlin, 1977, pp. 292-315. Lecture Notes in Math., Vol. 606.

[48] Y. Saad, M. H. Schultz, GMRES: a generalized minimal residual algorithm for solving nonsymmetric linear systems, SIAM J. Sci. Statist. Comput. 7 (3) (1986) 856-869. doi:10.1137/0907058. URL https://doi.org/10.1137/0907058 